\title{
The timing of the final assembly of the SNARE complex in exocytosis
}

\author{
$\mathrm{PhD}$ Thesis \\ In partial fulfillment of the requirements \\ for the degree "Dr. rer. nat." \\ in the Neuroscience Program \\ at the Georg-August-University Göttingen, \\ Faculty of Biology
}

submitted by

Alexander Matthias Walter

born in

Walsrode, Germany 


\section{Declaration}

I hereby declare that my $\mathrm{PhD}$ thesis 'The timing of the final assembly of the SNARE complex in exocytosis' has been written independently with no other aids or sources than quoted.

Göttingen, September $14^{\text {th }}, 2009$ 
To my grandfather, Leonard Palmer, the founder of my spirit for science 


\section{Contents}

1 Abstract …....................................................................................................... 1

2 Introduction............................................................................................................ 3

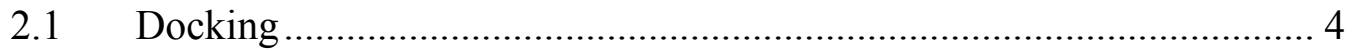

$2.2 \quad$ Vesicle Priming ............................................................................ 5

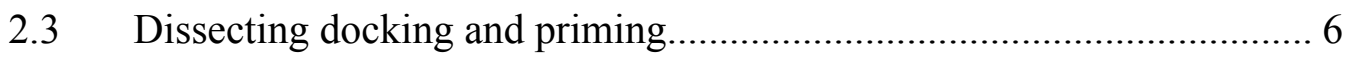

$2.4 \quad$ The parallel pool model …………………………………………….... 7

2.5 The role of the SNARE proteins in exocytosis ....................................... 9

2.6 Membrane fusion ............................................................................. 11

2.6.1 Characterization of the fusion pore ...................................................... 13

2.7 High speed fusion by stimulation from a high-energy state ………...... 13

2.8 A state with partial SNARE zippering as a possible high-energy

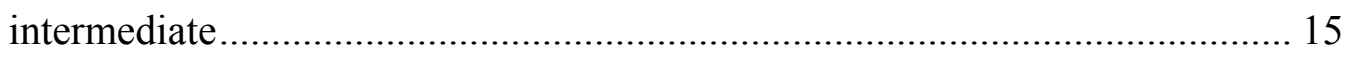

2.9 Mechanistic insights into SNARE action............................................ 16

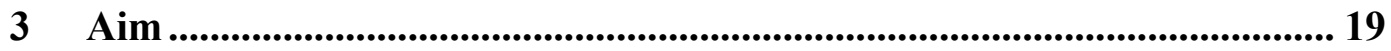

3.1 The scope of the thesis ...................................................................... 20

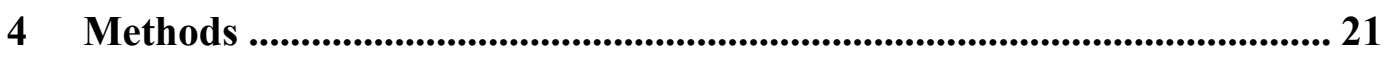

4.1 Electron microscopy of cultured chromaffin cells .............................. 21

$4.2 \quad$ Mutagenesis ……………………………………………….... 22

$4.3 \quad$ Protein biochemistry …………………………………………..... 23

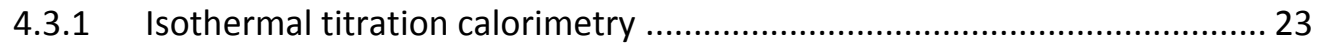

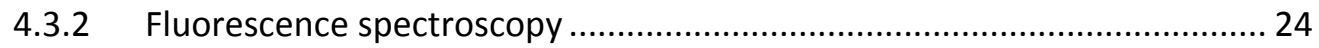

4.3.3 Circular dichroism spectroscopy …………………………………..... 24

4.4 Breeding of synaptobrevin 2/cellubrevin double knockout mice ......... 25

4.5 Electrophysiology and electrochemistry ............................................ 25

4.5.1 Membrane capacitance as an assay of exocytosis.................................. 26

4.5.2 Electrochemistry detects neurotransmitter efflux ............................. 27

4.5.3 Recording solutions ........................................................................... 29

4.5.4 $\mathrm{Ca}^{2+}$ photolysis and measurements of $\mathrm{Ca}^{2+}$ concentrations........................ 29

4.5.5 Kinetic analysis of capacitance responses................................................. 30

4.6 Immunocytochemistry …………………………………………..... 31 
4.7 Delineation of the Meyer Jackson model for pool kinetics ................... 32

4.7.1 The fusion pore forms essentially irreversibly ...........................................35

4.8 Derivation of Yet Even Another Hypothesis ("YEAH") .......................39

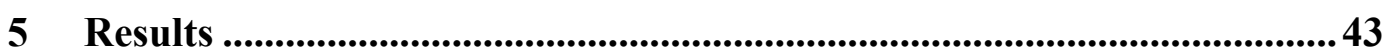

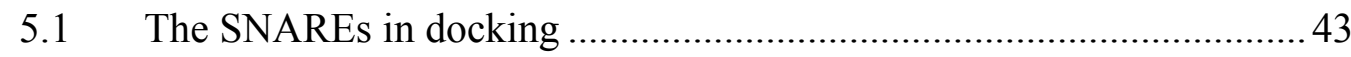

5.1.1 SNAP-25 is an essential docking factor ................................................ 44

5.1.2 Munc-18 promotes docking by stabilizing a syntaxin:SNAP-25 acceptor

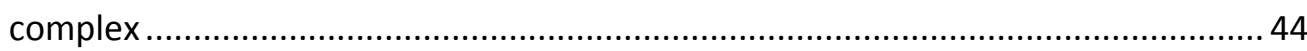

5.1.3 Synaptotagmin is a vesicular docking factor ......................................... 47

5.2 A partly assembled SNARE complex defines the primed vesicle.........54

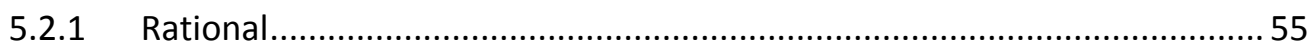

5.2.2 Mutants in synaptobrevin with regional destabilization.............................55

5.2.3 N-terminal destabilization affects vesicle priming .......................................60

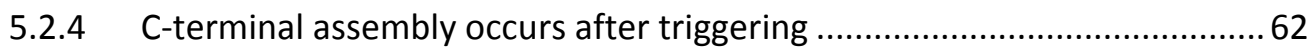

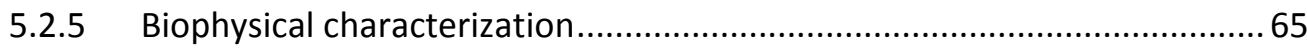

5.3 The C-terminal SNARE domain of synaptobrevin stabilizes the fusion

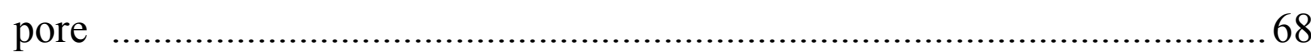

5.3.1 Fusion pore stability is reduced by C-terminal mutations ............................68

5.3.2 The Meyer Jackson proteinaceous pore model ........................................72

5.3.3 From single spikes to pool kinetics............................................................ 75

5.3.4 Towards concluding the pore ............................................................... 76

5.4 The main findings in a nutshell ........................................................ 76

5.4.1 The SNARE proteins and their binding partners act in docking .................77

5.4.2 N- to C-terminal SNARE zippering drives priming and fusion ..................... 77

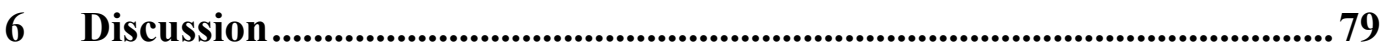

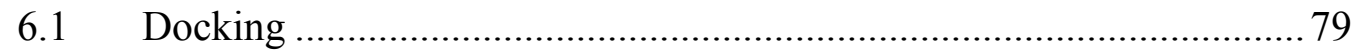

6.1.1 The role of the SNARE proteins in docking .............................................. 79

6.1.2 A model of the docking reaction in chromaffin cells................................. 81

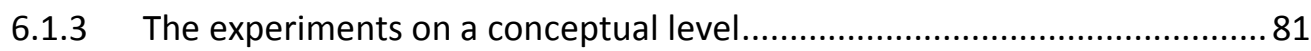

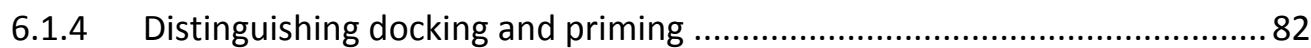

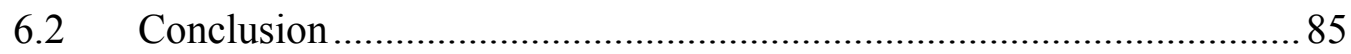

6.3 Sequential SNARE assembly comprises priming and triggering .......... 86

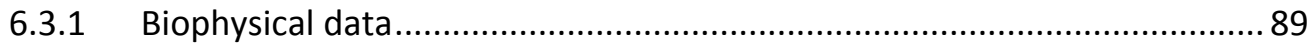




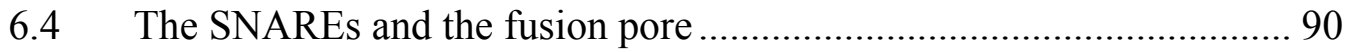

6.4.1 The C-terminal SNARE motif adds to the stability of the fusion pore......... 90

6.4.2 From single channels to pre-spike feet? ................................................. 91

6.5 Pool kinetics after SNARE destabilization ......................................... 93

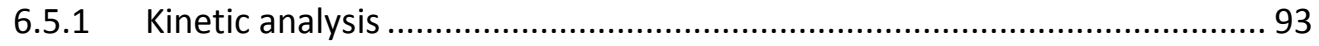

6.5.2 Yet Even Another Hypothesis ("YEAH") ....................................................... 95

6.5.3 Physiological interpretation of the fit parameters.................................... 97

6.5.4 Comparing the Voets parallel pool and the "YEAH" model ........................ 98

6.6 Discussion of the results on a conceptual level................................ 105

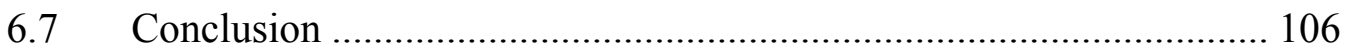

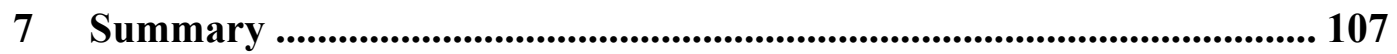

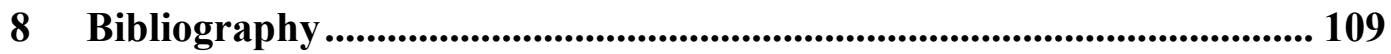

Acknowledgements....................................................................................................... 117

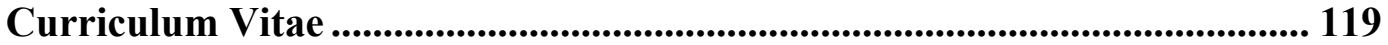

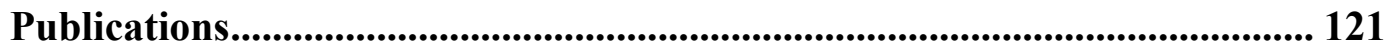





\section{Abstract}

Transfer of information between individual neurons via exocytosis is mediated by fusion of secretory vesicles with the plasma membrane and requires assembly of the SNARE proteins Syntaxin-1, SNAP-25 and Synaptobrevin 2 into a complex. Although the SNARE complex has been thoroughly studied in-vitro and many of its features including its structure have been characterized, little is known about the molecular mode of action in vivo. Above all, it remains to be established how action of the SNAREs mechanistically is involved in the steps necessary for synaptic vesicles to gain fusion competence (docking, priming) and how formation of the SNARE complex is involved in the final steps of fusion.

The present studies investigated the role of the SNARE proteins and their binding partners in docking, priming and fusion of secretory vesicles. As a model system, the mouse chromaffin cell enables the analysis of the role of individual proteins at the different stages of the release cycle in the clean genetic background of knockout mice. A number of different methodological approaches, ranging from electron microscopy to electrophysiological characterization, or mere biophysical techniques were utilized in order to generate a model of vesicle maturation and the molecular players involved therein.

It was found that SNAP-25 with the already known docking factors syntaxin and Munc-18 is essential for docking. Munc-18 may act by stabilizing a syntaxin:SNAP-25 docking platform required for stable association of the secretory vesicle to its release site, but its action is additionally crucial for subsequent priming. The vesicular docking factor was identified as synaptotagmin 1 , also known as the prime $\mathrm{Ca}^{2+}$ sensor for triggered release.

In order to further understand the exact molecular mechanism of SNARE complex assembly in the process of transmitter release, a mutagenesis approach was employed. Seeking to regionally weaken the SNARE complex, mutant variants of 
the vesicular SNARE protein Synaptobrevin 2 were introduced in knockout cells by viral overexpression and the secretory phenotype was characterized. A strong regio-sensitivity to SNARE destabilization with major effects of $\mathrm{N}$-terminal (membrane distal) destabilization on pool size but without effects on release kinetics was found. In contrast, C-terminal (membrane proximal) destabilization had a profound effect on the speed of transmitter release and, in addition, reduced the lifetime of the fusion pore. The regio-sensitivity observed agrees with a model of sequential SNARE complex assembly, in which N-terminal binding is involved in vesicle priming and $\mathrm{C}$-terminal binding is required for exocytosis triggering and fusion pore stability.

Together, these data, for the first time, allow the building of a model at the different stages of the vesicular release machinery in chromaffin cells with regard to docking, priming and fusion. 


\section{Introduction}

Neurotransmitter release is a process that occurs throughout the nervous system and is most crucial for information transduction (Sudhof, 2004). When signals are passed from cell to cell, an electrical signal is converted to a chemical one at a highly specialized interface, the synapse. Here, in response to membrane depolarization, voltage-gated $\mathrm{Ca}^{2+}$ channels are activated, resulting in an increase in intracellular $\mathrm{Ca}^{2+}$, the prime activator of neurotransmitter release. Transmitter discharge from the presynaptic cell is achieved when secretory vesicles fuse with the plasma membrane of the cell.

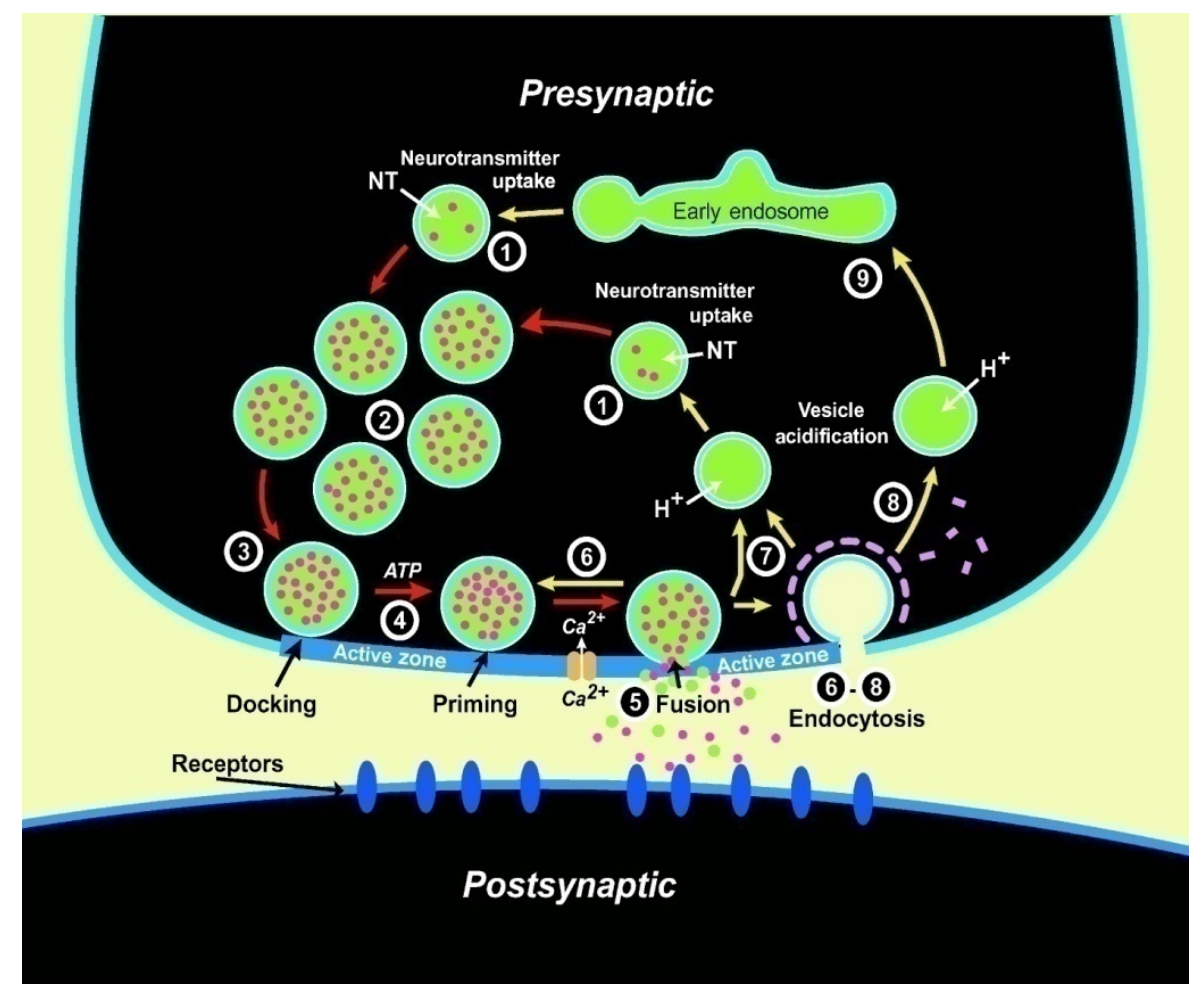

Figure 1. Schematic drawing of the vesicular release cycle at the neuronal pre-synapse. Adapted from (Sudhof, 2004).

Release occurs at high speed with high fidelity and reliability. It is thought that in order to account for these exquisite features of neurotransmission, the release 
process is organized into a cycle (Figure 1). Here, secretory vesicles are generated and filled with neurotransmitter (Figure 1,2). In an initial step vesicles become docked to the plasma membrane (Figure 1, 3). Then functional maturation of the vesicle is necessary in order to gain release competence, a process referred to as priming (Figure 1, 4). If now a $\mathrm{Ca}^{2+}$ stimulus triggers release, fusion of vesicles with the plasma membrane occurs, thereby releasing their content into the synaptic cleft (Figure 1,5). Subsequent diffusion of the transmitter to postsynaptic receptors and their activation allows for the initiation of a new signalling cascade in the postsynaptic cell. The cycle of secretory vesicles is not completed here, since vesicular membrane is retrieved by endocytosis (Figure 1, 6-8) and vesicles need to be re-acidified and re-filled with transmitter in order to become ready for a new round of release.

\subsection{Docking}

Docking is important for the process of exocytosis as it allows for initial spatial approximation of the vesicle to its release site. Docking is usually defined using morphological criteria, such as the distance from the plasma membrane measured in ultrastructural analysis. In this sense, a precise molecular definition of this process is as yet still missing (Verhage and Sorensen, 2008). Moreover, assessment of docked states varies widely throughout the literature due to different criteria in the definition. For instance, whereas some investigators consider vesicles to be docked when distances are less than $40 \mathrm{~nm}$ to the plasma membrane (Siksou et al., 2009), others argue that this distance should be $30 \mathrm{~nm}$ (de Wit et al., 2006). Yet again other studies consider vesicles without measurable distance to the plasma membrane (de Wit et al., 2009) as docked, whereas others require vesicles to touch and form a "patch" (Hammarlund et al., 2007).

Interestingly, the number of vesicles typically docked to the plasma membrane greatly exceeds the number of vesicles released by a brief stimulus, in agreement with an upstream role of docking in the maturation of vesicles and suggesting that docked vesicles consist of primed and un-primed vesicles. A number of genes 
have been identified that are important for vesicle docking (Verhage and Sorensen, 2008). Munc-18, a protein essential for neurotransmission at the synapse (Verhage et al., 2000) has also been reported to have an important function in the docking of secretory vesicles to the plasma membrane (Voets et al., 2001b). Munc-18 is an arc-shaped hydrophilic molecule that can bind to the neuronal SNARE complex (Deak et al., 2009; Dulubova et al., 2007) and to the plasma membrane protein syntaxin 1 in its "closed" conformation (Dulubova et al., 1999). Syntaxin 1, as one of the three SNARE (soluble $N$-ethylmaleimidesensitive factor attachment protein receptor) proteins is involved in the downstream process of neurotransmitter release. In addition, syntaxin also acts as a docking factor since its absence yields yet another severe docking phenotype (de Wit et al., 2006). Although these effects were initially discovered in chromaffin cells, the existence of similar defects has also been reported in other cells such as neurons of the nematode C.elegans (Hammarlund et al., 2007; Weimer et al., 2003). However, a discrepancy exists as to whether the closed or open form of syntaxin may be responsible for this action (Gerber et al., 2008; Gulyas-Kovacs et al., 2007; Hammarlund et al., 2007). The roles of Munc-18 and syntaxin 1 in the docking of secretory vesicles will be specifically addressed in due course.

\subsection{Vesicle Priming}

The next process in the vesicle cycle constitutes vesicle priming (Sudhof, 2004). After vesicles have been primed they can be rapidly released when exocytosis is triggered (Verhage and Sorensen, 2008). A priming step seems likely due to the fact that release in response to triggering can be extremely fast, too fast to account for a multi-step reaction (Sorensen, 2004). Moreover, the number of vesicles releasable by a $\mathrm{Ca}^{2+}$ stimulus only comprises a fraction of the vesicles docked (Rettig and Neher, 2002). Finally, upon repetitive stimulation, exocytosis slows down before the number of docked vesicles declines, suggesting that under these conditions priming and not docking constitutes the rate-limiting step (Sudhof, 1995). 
The original definition of priming was derived from work on permeabilized chromaffin cells, where the ability to secrete transmitters gradually decreases. Priming was defined as a MgATP and $\mathrm{Ca}^{2+}$-dependent mechanism restoring secretion (Holz et al., 1989). This process further depends on phosphatidylinositol-4,5-bisphosphate $\left(\mathrm{PIP}_{2}\right)$ and cis-SNARE complex disassembly (Verhage and Sorensen, 2008). This definition characterizes priming in reactions that experimentally occur on slow timescales and the involvement of additional factors here makes priming a feature that is not exclusive to the vesicle. According to a more recent, electrophysiological definition, priming refers to the maturation process by which a vesicle becomes readily releasable (Sorensen, 2004). Having undergone this reaction, fully matured vesicles contribute to the readily releasable pool, which is characterized by a very low release rate at resting cytosolic $\mathrm{Ca}^{2+}$-levels, but it dramatically accelerates as $\mathrm{Ca}^{2+}$ increases. The priming reaction itself appears to be $\mathrm{Ca}^{2+}$-dependent (Voets, 2000). In addition, priming can also be considered as a recruiting action, positioning the vesicles in proximity of $\mathrm{Ca}^{2+}$-channels (Neher and Sakaba, 2008). Indeed, recent evidence suggests that synaptotagmin may act in the speed-up of release by facilitating positional priming (Young and Neher, 2009).

\subsection{Dissecting docking and priming}

The distinction between docking and priming reactions is difficult. Although a priming step seems likely to explain the physiological observations, there is little experimental evidence that priming and docking are mutually distinct reactions. Evidence that these reactions are distinct can be drawn from genetic alterations that selectively affect priming without effect on docking. Such conclusions were drawn from experiments on Munc-13 or CAPS deficient organisms. In the absence of Munc-13, priming was thought to be affected without apparent effect on vesicle docking (Brose et al., 2000). However, in a recent study utilizing sophisticated high pressure freezing techniques, a docking defect could also be detected, suggesting that docking and priming may be representations of an 
identical process (Siksou et al., 2009). Similar confusion exists as to the role of calcium-activator protein for secretion (CAPS). CAPS was shown to reduce the size of the readily releasable pool in chromaffin cells and therefore implicated in vesicle priming without effect on vesicle docking (Liu et al., 2008). In contrast, recent data from C.elegans do show an effect on dense core vesicle docking (Hammarlund et al., 2008). The authors of the latter study further identify a systematic difference between dense core vesicle docking and docking of synaptic vesicles.

Some evidence suggests that several different states in docking exist that can be distinguished by their mobility (Nofal et al., 2007) or the residence time and tethering force (Toonen et al., 2006). It was found that only the most immobile and most strongly tethered vesicles can be activated for fusion, consistent with the idea that a docking reaction proceeds priming and these reactions are linearly coupled (Rettig and Neher, 2002).

\subsection{The parallel pool model}

Different kinetic pools are characterized by the speed at which ensembles of vesicles can undergo fusion. These differences can, in principle, be due to a difference in localization of the secretory vesicle and therefore differences in apparent $\mathrm{Ca}^{2+}$-levels. Alternatively they could be due to differences on a molecular level with different intrinsic $\mathrm{Ca}^{2+}$-sensitivities. In order to distinguish between these two possibilities, Voets and colleagues performed experiments in which stimulation was performed by voltage depolarization as opposed to flashevoked $\mathrm{Ca}^{2+}$-uncaging: voltage depolarization of the cell leads to the opening of $\mathrm{Ca}^{2+}$ channels, allowing for influx of $\mathrm{Ca}^{2+}$, but its concentration will be inhomogeneous throughout the cell; vesicles in close proximity to $\mathrm{Ca}^{2+}$ channels will be exposed to higher levels while vesicles at a greater distance will sense the increase with a delay. This effect can be circumvented by application of a stimulus that, throughout the cell, establishes homogeneous $\mathrm{Ca}^{2+}$ levels, such as application of a UV flash, capable of photolysing a $\mathrm{Ca}^{2+}$ chelator (Voets et al., 
1999). Comparison of kinetic data obtained by both these types of experiments indeed established a difference in release kinetics due to a positional effect. However, in addition, even in the flash experiment, two different time constants of release were resolved, in agreement with the notion that different pools show a molecular heterogeneity (Voets et al., 1999). This distinction was based on the observation that in exocytic bursts cellular capacitance increase followed biexponential behavior and thus gave rise to the assumption of two parallel pools (Figure 2) (Voets, 2000): the slowly releasable pool (SRP) and the readily releasable pool (RRP) (Voets et al., 1999). This is different from recent data obtained in neurons. At the calyx of Held flash-evoked exocytosis revealed a convergence, though not perfect, of the fast and slow pool, due to speed-up of the latter. This suggests a difference on the positional rather than on the molecular level (Wadel et al., 2007). However, other experiments at the calyx using flashevoked release also revealed a difference in release probability due to heterogeneous $\mathrm{Ca}^{2+}$-sensitivity (Wolfel et al., 2007).

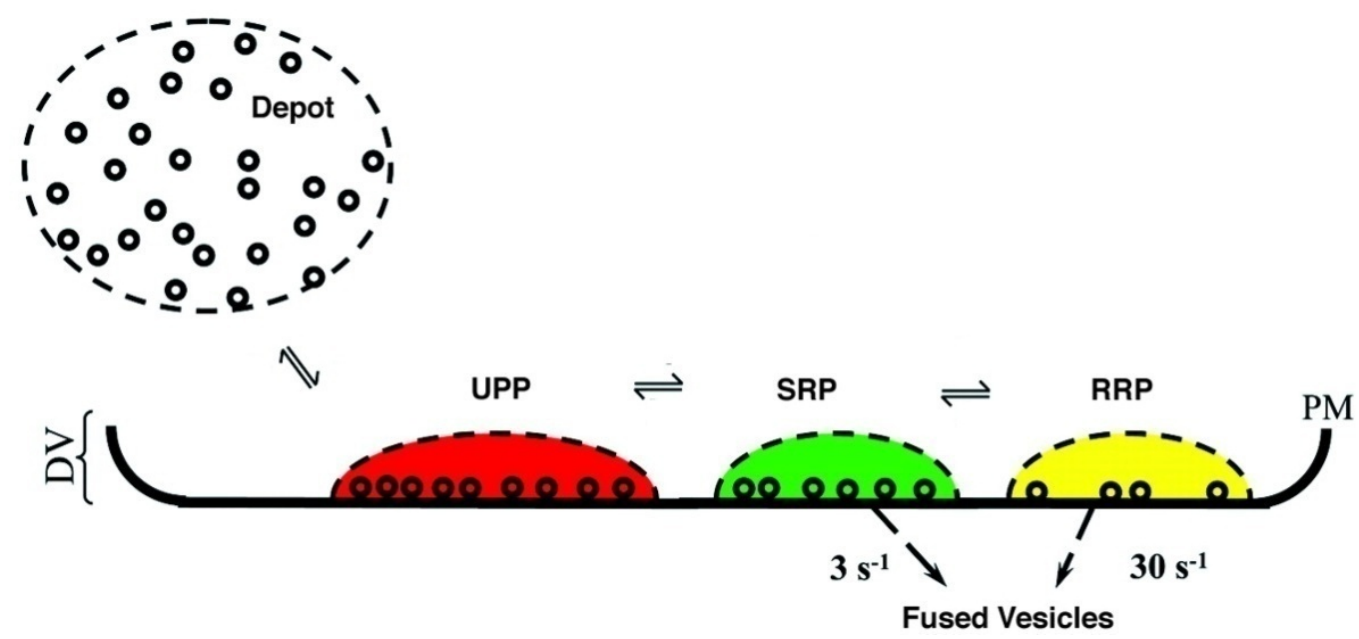

Figure 2. The parallel pool model (PPM) of release in chromaffin cells. Vesicles are depicted in four different states. Vesicles of the depot pool enter the docked, but unprimed pool (UPP). Releasable vesicles are subdivided into the slowly releasable pool (SRP) and the readily releasable pool (RRP) which can fuse directly in triggered release. Abbreviations: DV, docked vesicles; PM, plasma membrane. Modified from Rettig and Neher (2002).

The vesicles residing in the releasable pools are distinct from the ones constituting the reserve pool which need to undergo priming in order to become releasable. In 
chromaffin cells, a sequential, linear model of these processes has been suggested (Voets, 2000). So far, the molecular state of the primed vesicle, which is assumed to be a high energy intermediate between vesicles docked to the plasma membrane and vesicles undergoing fusion in triggered exocytosis, remains elusive. Much of the work presented here deals with the identification and characterization of the primed state.

After $\mathrm{Ca}^{2+}$ stimulation, exocytosis is rapid and release occurs on the timescale of microseconds. Much of the current research is addressed towards the identification of the mediators of this process, which involves synaptotagmin 1 as the prime $\mathrm{Ca}^{2+}$ sensor of exocytosis (Geppert et al., 1994; Sorensen et al., 2003a; Voets et al., 2001a), possibly interacting with complexin (Maximov et al., 2009; Sudhof and Rothman, 2009).

\subsection{The role of the SNARE proteins in exocytosis}

It is only with high energy costs that it is possible to fuse membranes of vesicle and target since electrostatic repulsive and hydration forces need to be overcome (Zimmerberg et al., 1993). The energy necessary to promote this action is thought to be provided by the formation of a stable complex, consisting of the SNARE proteins syntaxin 1, SNAP-25 (synaptosome-associated protein of $25 \mathrm{kDa}$ ) and synaptobrevin 2, also known as VAMP2 (vesicle-associated membrane protein). The SNARE complex is extremely heat stable, resistant to SDS denaturation, (Chen and Scheller, 2001) and assembly of only few complexes may provide sufficient energy for the promotion of fusion (Li et al., 2007; Wiederhold and Fasshauer, 2009).

SNARE proteins are a family of membrane proteins that can form heterooligomeric complexes. Their binding motif consists of homologous coiled coil regions, arranged in heptad repeats and comprised of approximately 60 residues, the so-called SNARE motif (Fasshauer, 2003). Most of the SNARE proteins are attached to the membrane by a C-terminal transmembrane domain connected by a 
short linker (Kloepper et al., 2007). SNARE complex formation is thought to occur after the initial interaction of the N-terminal side of the SNARE proteins followed by $\mathrm{N}$ - to C-terminal zippering, thereby bringing membranes into close approximation. The complex contains 16 highly conserved layers, essentially adding to its stability through mostly hydrophobic interaction. However, a characteristic central hydrophilic layer is formed by the respective residues contributed by the individual SNARE partners. Here, three glutamines are contributed by the three Q SNAREs $\left(\mathrm{Q}_{\mathrm{a}}, \mathrm{Q}_{\mathrm{b}}, \mathrm{Q}_{\mathrm{c}}\right)$ and an arginine is added by the $\mathrm{R}$ SNARE. SNARE complexes are therefore characterized by the formation of a coiled coil complex of the four $\mathrm{Q}_{\mathrm{abc}} \mathrm{R}$ domains (Fasshauer, 2003). Although SNARE proteins have been found to be essential for fusion and transport of numerous reactions in eukaryotic cells, in the work presented here merely the neuronal SNARE proteins SNAP-25, syntaxin 1 and synaptobrevin 2 will be addressed.

The structure of the neuronal SNARE complex has been solved by X-ray crystallography (Stein et al., 2009; Sutton et al., 1998). The four-helical coiled coil complex is established by each one alpha-helix of syntaxin 1 and synaptobrevin 2 as well as two helices of SNAP-25. The neuronal SNARE complex is therefore exceptional with regard to its stoichiometry in the sense that two domains are added to the complex by a single protein, SNAP-25 - a unique feature, which may have evolved specifically to meet the demands necessary for neurotransmitter release (Nagy et al., 2008). The SNARE proteins syntaxin and synaptobrevin 2 each contain a transmembrane domain at the C-terminus, whereas SNAP-25 becomes membrane-associated by palmitoylation of cysteins within the linker region.

It is well established that the SNARE proteins represent essential and sufficient factors for membrane fusion in-vitro (Holt et al., 2008; Weber et al., 1998), impressively demonstrated by the severe effects of toxins acting on SNAREs and on their ability to fuse membranes when reconstituted into liposomes.

Detailed studies in-vitro have added much to the understanding of the function of SNAREs in membrane fusion. However, two aspects of exocytosis in the cell 
have thus far not been successfully reproduced in in-vitro assays: speed and timing. Under physiological conditions, neurotransmitter release occurs in the range of microseconds and is precisely controlled by a $\mathrm{Ca}^{2+}$ stimulus. This process is referred to as exocytosis triggering and is believed to require interaction of a $\mathrm{Ca}^{2+}$ sensor. Much evidence exists that this role is attributed to the vesicular protein synaptotagmin (Chapman, 2008; Geppert et al., 1994; Rizo et al., 2006; Sorensen et al., 2003a; Voets et al., 2001a), although to date it is not clear whether this action requires interaction with the SNARE complex (Rickman and Davletov, 2003), with the lipids (Xue et al., 2008) or both (Dai et al., 2007; Lynch et al., 2008). Although it has been shown that synaptotagmin binds to proteins of SNARE complex (Rickman and Davletov, 2003) and this binding is implicated in release, synaptotagmin also has the special feature of being capable of inducing positive membrane curvature (Martens et al., 2007). Synaptotagmin may therefore also facilitate fusion by lowering the activation barrier and stabilizing high energy intermediates of the fusion reaction.

\subsection{Membrane fusion}

Vesicle fusion in triggered exocytosis involves the fusion of the vesicular and the target membrane. This reaction requires close proximity, membrane merging and the opening of an aqueous fusion pore. According to the Stalk hypothesis (Figure 3, a), this requires transient non-bilayer intermediates (Jahn et al., 2003), which are high energy states characterized by high membrane curvature, delimited by forces that reduce the exposure of hydrophobic surfaces to water: proximal membranes fuse first, forming a highly bent stalk (Figure 3, a2), whereas the distal membranes remain continuous. The distal membranes are bent toward one another. These intermediates are also referred to as hemifused states (Figure 3, a3) (Jahn and Sudhof, 1999). In this model, further reaction occurs when discontinuities in the inner leaflet lead to formation of a lipidic fusion pore, connecting the lumen of the vesicle with the extracellular space (Figure 3, a4). Expansion of the fusion pore completes vesicular fusion (Figure 3, a5). 
According to an alternative hypothesis by Meyer Jackson and colleagues, the fusion pore is lined by the SNARE proteins (Figure 3, b), producing a configuration similar to an ion channel (Figure 3, b3/b4) (Jackson and Chapman, 2006), in whose expansion synaptotagmin also plays a role (Wang et al., 2006; Wang et al., 2003).

The involvement of the SNARE proteins in fusion pore dynamics will be addressed in this thesis, as will be the compliance with the Meyer Jackson proteinaceous pore model.
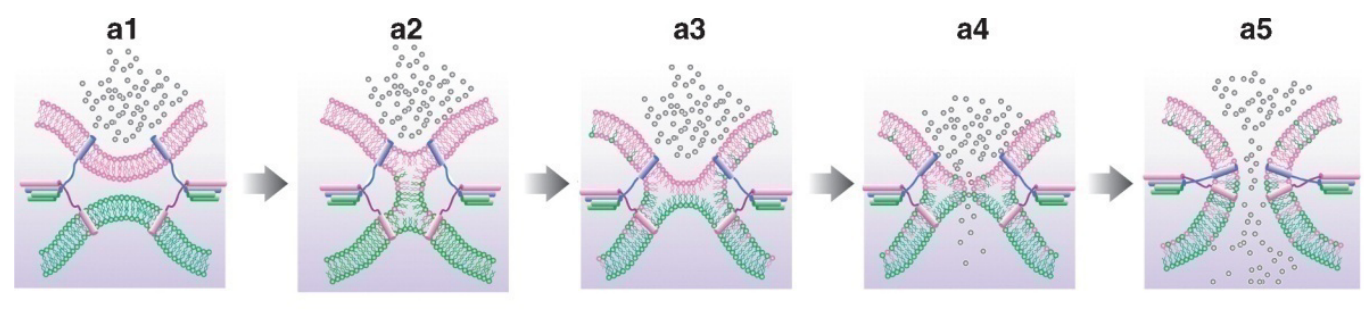

b3
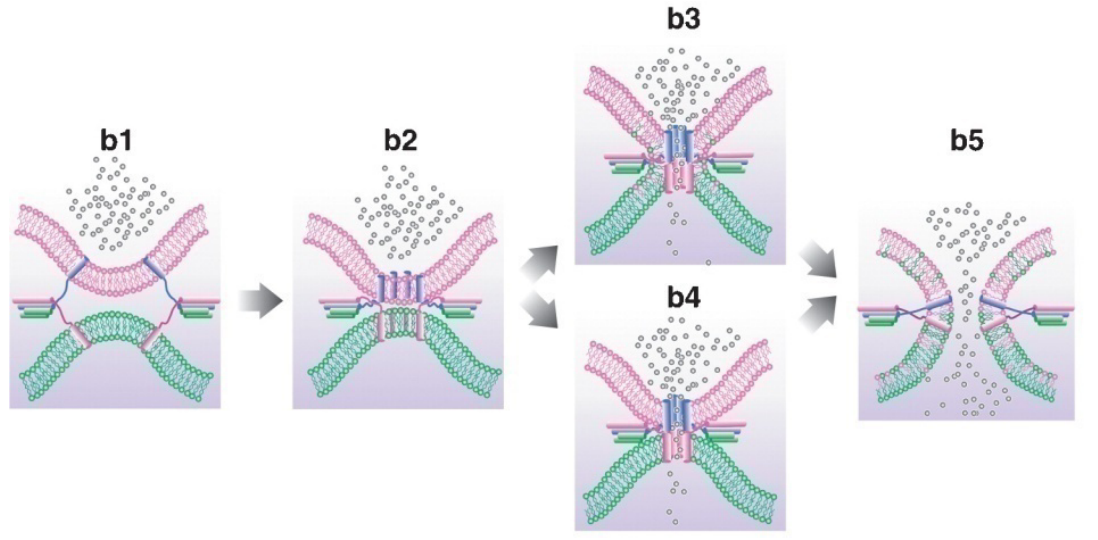

Plasma membrane Vesicle membrane Neurotransmitter

Syntaxin

$\checkmark$ SNAP-25

$\checkmark$ Synaptobrevin II

Figure 3. Models for membrane fusion. According to the Stalk hypothesis (a), close proximity of the membranes leads to fusion of the proximal monolayers producing a stalk (a2). From the hemifused state (a3) fusion pores form, which are of lipidic nature (a4), expansion of the pore leads to full fusion.

According to the protein-pore model (b), the fusion pore is lined by the transmembrane domains of the SNARE proteins $(\mathbf{b 3}, \mathbf{b 4})$, either in a state of bilayers (b4), or in a state surrounded by a hemifused leaflet (b3). Fusion pore expansion occurs when the transmembrane domains separate laterally and lipids are incorporated into the pore. The bilayers merge (b5). Adapted from Jackson and Chapman (2006). 


\subsubsection{Characterization of the fusion pore}

Although the precise structure of the fusion pore in regulated exocytosis remains elusive, electrophysiological characterization using a variety of techniques has added much to the understanding of the dynamics of the pore (Lindau and Alvarez de Toledo, 2003). For instance, amperometric recordings allow measuring the efflux of transmitter as it is being released from neurosecretory cells such as chromaffin cells and PC-12 cells. The temporal resolution in these measurements allows for the detection of so-called "foot signals", which often precede the amperometric spike. It was originally shown in Mast cells that the foot signal is caused by release of vesicular content through the fusion pore (Alvarez de Toledo et al., 1993). This method therefore allows for the characterization of fusion pore lifetime by measurement of pre-spike foot duration and has been widely applied to the characterization of fusion pore properties following genetic manipulations (Borisovska et al., 2005; Han and Jackson, 2006; Kesavan et al., 2007; Sorensen et al., 2003b; Wang et al., 2006; Wang et al., 2001; Wang et al., 2003) and in thermodynamic characterization of the fusion reaction (Zhang and Jackson, 2008). Complementary to this technique, cell-attached capacitance measurements can be performed, allowing the characterization of secretory vesicle size by measurement of the vesicular capacitance and direct measurement of the fusion pore conductance (Lindau and Alvarez de Toledo, 2003). This method has also been used to study properties of synaptic vesicles (He et al., 2006). Combined amperometric and capacitance measurements by means of patch amperometry allow for simultaneous measurement of cell-attached membrane capacitance and transmitter release (Albillos et al., 1997).

\subsection{High speed fusion by stimulation from a high-energy state}

In the light of the rapid release of primed vesicles following exocytosis triggering, activation of release from a high-energy intermediate state appears likely. A proposal is the idea that the vesicle is clamped in a ready-to-go configuration from which fusion can be rapid when exocytosis is triggered. It is generally accepted 
that in order to account for these features in vivo additional proteins as well as the SNAREs are involved. These may include the complexins and synaptotagmins (Sudhof and Rothman, 2009). Complexins are proteins that can tightly bind to the C-terminal portion of the assembled four-helical SNARE complex (Chen et al., 2002). It could therefore interfere with fusion by acting as a clamp or facilitate the reaction by stabilizing a key intermediate (Rizo and Rosenmund, 2008). From experiments in-vitro, using "flipped" SNAREs, SNAREs that are ectopically expressed, it has been implied that complexin acts by clamping the fusion mechanism, since it reduced the rate of cell-cell fusion which occurred constitutively with flipped SNAREs. Addition of synaptotagmin 1 increased the fusion rate in the presence of complexin in a $\mathrm{Ca}^{2+}$-sensitive manner, leading to the speculation that synaptotagmin acts as an activator of fusion by displacing complexin (Giraudo et al., 2006; Giraudo et al., 2009). Other approaches in-vitro, utilizing liposome fusion assays and the cytoplasmic domain synaptotagmin, implied that the $\mathrm{Ca}^{2+}$-dependent speed-up of fusion was dependent on synaptotagmin itself, not by clamp removal, but by mere facilitatory action and $\mathrm{Ca}^{2+}$-sensitive SNARE-interaction (Tucker et al., 2004). However, this model has been challenged recently by analysis of full-length synaptotagmin (Stein et al., 2007) and to date does not allow for a conclusive deduction of the role of these proteins in vivo.

In vivo experiments have suggested a role of complexin in clamping premature release, thus stabilizing the vesicle in a ready-to-go configuration (Maximov et al., 2009). Competitive binding of synaptotagmin and complexin to SNAREs have been implicated as an underlying mechanism of exocytosis triggering by synaptotagmin dependent complexin displacement and clamp removal (Tang et al., 2006). Yet, this idea is in conflict with a reported positive action of complexin II on events upstream of exocytosis triggering in chromaffin cells (Cai et al., 2008), a decreased release probability in complexin I/II double knockout cells (Reim et al., 2001) and a reported complementary, rather than synergistic action of the two proteins (Xue et al., 2007). 


\subsection{A state with partial SNARE zippering as a possible high- energy intermediate}

Despite these controversies, the establishment of a vesicle in a ready-to-go configuration appears as a likely explanation for the specific features required in the exquisite process of triggered exocytosis. This might comprise a state of partial SNARE complex assembly. Evidence exists that the initial binding of synaptobrevin 2 to a syntaxin:SNAP-25 acceptor complex is mediated via the Nterminus of synaptobrevin 2 (Pobbati et al., 2006; Sorensen et al., 2006). A partly assembled complex would therefore correspond to a synaptobrevin N-terminally bound to the SNARE complex, but not attached via its C-terminal SNARE motif. Support for the existence of such a species can be deduced from studies with toxins in neuronal and acrosomal fusion (De Blas et al., 2005; Hua and Charlton, 1999; Xu et al., 1998; Xu et al., 1999).

A previous study applying mutagenesis to SNAP-25 supported the notion of sequential SNARE complex assembly in the process of priming and triggering of neurotransmitter release. Here, C-terminal destabilization of SNARE interaction interfered with the speed of release, whereas mutation in the middle of the complex interfered with poolsize, an effect attributed to a defect in priming (Sorensen et al., 2006). However, considering the sequence of steps leading to the formation of the SNARE complex it cannot be excluded that SNAP-25 manipulation interfered with upstream reactions of syntaxin:SNAP-25 acceptor complex formation or even vesicle docking. Indeed, recently the role of SNAP-25 in docking was reinvestigated, establishing a crucial role of SNAP-25 (de Wit et al., 2009). In the light of these findings, the previous study needs to be reconsidered.

Much of the work presented here deals with the characterization of secretion and the dissection of priming and triggering following mutation of the vesicular SNARE protein synaptobrevin 2. Analysis of mutants in the genetic background of the synaptobrevin 2/cellubrevin double knockout mouse (Borisovska et al., 2005) enables one to study the attachment of the vesicular counterpart to the syntaxin:SNAP-25 acceptor complex in isolation. The timing of synaptobrevin 
binding to the acceptor complex as well as the correlation between SNARE complex assembly and fusion were investigated.

The work presented here establishes the role of the N-terminal part of the vesicular SNARE protein synaptobrevin 2 in the formation of the primed state as well as the C-terminal end in fusion triggering and fusion pore stability.

\subsection{Mechanistic insights into SNARE action}

Since the discovery of the SNARE proteins and their requirement for membrane fusion reactions much has been learned about the fundamental processes of neurotransmission and exocytosis. Currently, a vast number of factors involved are known and the network of underlying reactions in the processes of docking, priming and triggering gains more complexity. In spite of increasing genetic evidence linking these proteins in their action, little fundamental mechanistic insights exist of how these machineries work on a molecular level. Especially, the current understanding is insufficient to create a concise model of the elementary reactions involved in exocytosis. For instance, the factors involved in docking and the fundamental mechanisms of this reaction remain elusive, as is the link between docking and the subsequent priming reaction. Both states lack a molecular definition. Docking is vaguely defined by morphological criteria due to the various distinctions used in the literature and dependent on the fundamental design of an experiment, its conditions of fixation and the respective model system. Priming on the other hand, defined by physiological criteria, depends on the nature of the preparation as well as stimulation protocols. Much of the work presented here deals with the molecular characterization of these states and the synthesis of a model linking the reactions. Especially, understanding the sequence and the precise timing of the individual reactions is important for appreciating the relevance of each step of the fusion process.

Genetic manipulations aid the isolation and individual characterization of reactions in the release cycle. The model system of the mouse chromaffin cell 
allows the investigation of morphology as well as the analysis of secretion electrophysiologically with high temporal resolution. A combination of these experiments with the biochemical investigation of protein interaction and mathematical modeling greatly increases our understanding of the molecular mechanisms and energy profile of the essential reaction underlying all neurotransmission. 


\section{Aim}

The work of the study presented here was concerned with the characterization of the events involved in docking, priming and triggering of exocytosis. Especially, the identification of the factors involved in the respective reactions as well as their interaction partners was of particular interest. Additionally, the question whether priming and docking can indeed be clearly distinguished, as would be expected in a linear, sequential model was addressed. Particular attention was given to the identification of the primed vesicle state. The hypothesis of sequential SNARE complex assembly as previously proposed (Sorensen et al., 2006) was tested using a mutagenesis approach. Ultimately, the work presented here is devoted to the building of a minimal working model for the reactions involved in docking, priming and triggering of exocytosis. 


\subsection{The scope of the thesis}

- Check the involvement of the SNARE proteins in docking by combining morphological characterization of docking and electrophysiological characterization of secretion

- Characterization of Munc-18 knockout cells

- Study of interaction of Munc18 and SNAP-25 by cross-rescue approaches

- Investigation of syntaxin1:SNAP-25 interaction as a putative acceptor complex for a vesicular docking factor

- Characterization of synaptotagmin deficiency and its effect on docking

- Test the hypothesis of sequential SNARE complex assembly by electrophysiological and electrochemical characterization of secretion and by studying protein interaction biochemically

- Mutagenesis of synaptobrevin 2 and expression of mutant variants in synaptobrevin/cellubrevin double knockout cells to test for regiosensivity

o Biophysical characterization of mutant SNARE complexes with respect to thermal stability and ternary structure, binding kinetics and thermodynamics

- Characterization of single vesicle fusion by means of carbon fiber amperometry following regional SNARE complex destabilization

- Delineation of a concise working model 


\section{Methods}

\subsection{Electron microscopy of cultured chromaffin cells}

Adrenal glands were removed at embryonic day 18 and cultured as described previously (Sorensen et al., 2003b). Chromaffin cells from synaptotagmin-1 or munc18-1 null, littermate controls (E18), and wild type mice were plated on rat tail type 1 collagencoated (32 $\mu \mathrm{g} / \mathrm{ml}$; Beckton Dickinson labware, USA) coverslips (Cellocate, Eppendorf, Germany) and infected (DIV2, 2 days in-vitro) with semliki forest virus (SFV) constructs. Cells were observed under a fluorescence microscope $6 \mathrm{~h}$ after infection and the location of infected/control cells was mapped. Cells were fixed for $45 \mathrm{~min}$ at room temperature with $2.5 \%$ glutaraldehyde in 0.1 M cacodylate buffer (pH 7.4) (Toonen et al., 2006). After fixation cells were washed with $0.1 \mathrm{M}$ cacodylate buffer ( $\mathrm{pH} 7.4$ ), post-fixed for $2 \mathrm{~h}$ at room temperature with $1 \% \mathrm{OsO} 4$ in bidest, washed and stained with $1 \%$ uranyl acetate for $40 \mathrm{~min}$ in the dark. Following dehydration through a series of increasing ethanol concentrations, cells were embedded in Epon and polymerized for $24 \mathrm{~h}$ at $60^{\circ} \mathrm{C}$. The coverslip was removed by alternately dipping in liquid nitrogen and hot water. Cells of interest were selected by observing the flat Epon embedded cell monolayer (containing the gridded Bellco print) under the light microscope, and mounted on pre-polymerized Epon blocks for thin sectioning. Ultra thin sections ( $\sim 90 \mathrm{~nm}$ ) were cut parallel to the cell monolayer and collected on single-slot, formvar-coated copper grids, and stained with uranyl acetate and lead citrate. Analysis of secretory vesicle distribution was done blind for the genotype of the animal. For each condition the distribution of secretory vesicles was analyzed in serial ultrathin sections $(\sim 90 \mathrm{~nm})$ of randomly selected chromaffin cells from different animals (and three different grids per animal). Chromaffin cells were selected at low magnification in the JEOL 1010 electron microscope and subsequently examined at 20.000x magnification. Only cells with a visible nucleus and clear-cut plasma membrane were taken into account. Secretory vesicles were recognized by their round, dense core and had a diameter 
of approximately $90 \mathrm{~nm}$. Docked vesicles were without any measurable distance between granule and plasma membrane. Distances from the granule membrane to the plasma membrane were measured on digital images acquired at $20,000 x$ magnification by a Kodak MegaPlus $1.4 \mathrm{i}$ camera controlled by analySIS (Soft Imaging Systems / Olympus, Germany). For each condition the relative frequency of docked vesicles was calculated, and cumulative distance histograms were created of the relative frequency of all vesicles according to the number of vesicles counted in this area. Electron microscopic data are presented as mean \pm SEM, and statistical significance among various groups was evaluated with Student's t-test.

\subsection{Mutagenesis}

Mutations were introduced into synaptobrevin 2 (rat, syb 2) and synaptotagmin 1 (syt 1) within a pSFV1 plasmid using PCR mutagenesis with primers that contained a mismatch introducing a point mutation or that omitted a part of the sequence to introduce a deletion. Syb 2 and syt 1 were excised from the plasmid using BamHI and BssHII restriction sites and mutants were generated by two subsequent rounds of PCR: initially two fragments of the syb 2/syt 1 DNA were generated: (1) from the BamHI restriction site followed by the start codon to the site of the mutation and (2) from the site of the mutation to the stop codon and the following BssHII restriction site. In a second PCR reaction the two fragments were fused by addition of the syb 2/syt 1 forward and reverse primes after one initial PCR reaction. All constructs contained a Kozak sequence in front of the start codon and all mutants were verified by subsequent sequencing. In the pSFV1, the syb 2/syt 1 ORF was followed by an Internal Ribosomal Entry Site (IRES) and EGFP for later identification of infected cells in the experiment. Preparation of virus particles was performed as described (Ashery et al., 1999). 


\subsection{Protein biochemistry}

The basic SNARE expression constructs, cysteine-free SNAP-25A (res. 1-206), the syntaxin 1A SNARE motif (SyxH3, res. 180-262), the soluble domain of synaptobrevin 2 (Syb1-96) and a shortened synaptobrevin construct, syb49-96, have been described before (Fasshauer et al., 2002; Fasshauer and Margittai, 2004; Pobbati et al., 2006). In addition, various synaptobrevin mutants and truncations were used and single cysteines were introduced at position 28 for labeling purposes: Syb1-96 L32A, T35A, S28C; Syb1-96 V39A, V42A, S28C; Syb1-96 L84A, S28C; Syb1-96 L84N, S28C; Syb1-96 A32-35, S28C; Syb1-96 432-35, V39A, V42A, S28C. They were cloned into the pET28a vector (Novagen) via Nde1 and Xho1 sites, resulting, after cleavage of the His6-tag by thrombin, in the additional N-terminal sequence GSHM. All proteins were expressed in Escherichia coli strain BL21 (DE3) and purified by $\mathrm{Ni}^{2+}-\mathrm{NTA}$ chromatography followed by ion exchange chromatography on an Äkta system (GE Healthcare) essentially as described (Fasshauer et al., 1999). His6-tags were generally removed using thrombin. All SNARE complexes were purified using a Mono-Q column (Pharmacia) after overnight assembly of the purified monomers. The following ternary complexes were employed: Syb49-96:SyxH3:SNAP-25 $(\Delta \mathrm{N}$ complex), and Syb1-96S28C mutants:SyxH3:SNAP-25. Protein concentrations were determined by absorption at $280 \mathrm{~nm}$ in $6 \mathrm{M} \mathrm{GdnHCl}$ and/or using the Bradford assay.

\subsubsection{Isothermal titration calorimetry}

Isothermal titration calorimetry (ITC) was performed on a VP-ITC instrument (Microcal) at $25{ }^{\circ} \mathrm{C}$ essentially as described (Burkhardt et al., 2008). Samples were dialyzed twice against degassed phosphate buffer $(20 \mathrm{mM}$ sodium phosphate, $\mathrm{pH} 7.4,150 \mathrm{mM} \mathrm{NaCl}, 1 \mathrm{mM}$ DTT). Typically, an initial $5 \mu$ injection was followed by several $15 \mu 1$ injections. The heat change per injection was integrated to yield the molar enthalpy for each injection. Blank titrations, which were carried out by injection ligand into buffer, were subtracted from each data set. All ITC experiments were carried out at least twice. The resulting binding 
isotherms were analyzed using the Microcal Origin ITC software packet to obtain the binding enthalpy $(\Delta \mathrm{H})$, the stoichiometry $(\mathrm{n})$, and the association constant $\left(\mathrm{K}_{\mathrm{A}}\right)$. A one site binding model was used that assumes that one or more ligands can bind independently. The dissociation constant $\left(\mathrm{K}_{\mathrm{D}}\right)$ and the binding free energy $(\Delta G)$ were calculated using the basic thermodynamic relationships $K_{D}=$ $\mathrm{K}_{\mathrm{A}}{ }^{-1}, \Delta \mathrm{G}=-\mathrm{RT} \ln \mathrm{K}_{\mathrm{A}}$, and $\Delta \mathrm{G}=\Delta \mathrm{H}-\mathrm{T} \Delta \mathrm{S}$.

\subsubsection{Fluorescence spectroscopy}

All measurements were carried out in a Fluorolog 3 spectrometer in $\mathrm{T}$ configuration equipped for polarization (Model FL322, Horiba Jobin Yvon). Single cysteine variants were labelled with Alexa488 C5 maleimide according to the manufacturer's instructions (Invitrogen). All experiments were performed at $25{ }^{\circ} \mathrm{C}$ in $1 \mathrm{~cm}$ quartz cuvettes (Hellma) in phosphate buffer $(20 \mathrm{mM}$ sodium phosphate, $\mathrm{pH} 7.4,150 \mathrm{mM} \mathrm{NaCl}, 1 \mathrm{mM}$ DTT). Measurements of fluorescence anisotropy, which reports the local flexibility of the labeled residue and which increases upon complex formation and decreases upon dissociation, were carried out essentially as described (Fasshauer and Margittai, 2004; Pobbati et al., 2006). The $G$ factor was calculated according to $G=I_{H V} / I_{H H}$, where $I$ is the fluorescence intensity, and the first subscript letter indicates the direction of the exciting light and the second subscript letter the direction of emitted light. The intensities of the vertically $(\mathrm{V})$ and horizontally $(\mathrm{H})$ polarized emission light after excitation by vertically polarized light were measured. The anisotropy (r) was determined according to $r=\left(I_{V V}-G I_{V H}\right) /\left(I_{V V}+2 G I_{V H}\right)$.

\subsubsection{Circular dichroism spectroscopy}

CD measurements were performed essentially as described (Fasshauer et al., 2002; Fasshauer and Margittai, 2004; Pobbati et al., 2006) using a Chirascan instrument (Applied Photophysics). Hellma quartz cuvettes with a pathlength of $0.1 \mathrm{~cm}$ were used. The measurements were carried out at $25^{\circ} \mathrm{C}$. For thermal denaturation experiments, the purified complexes were dialyzed against phosphate 
buffer. The ellipticity at $222 \mathrm{~nm}$ was recorded between 25 and $95^{\circ} \mathrm{C}$ at a temperature increment of $30^{\circ} \mathrm{C} / \mathrm{h}$.

\subsection{Breeding of synaptobrevin 2/cellubrevin double knockout mice}

The mice were kept in two separate colonies. The cellubrevin (ceb) colony was continuously crossed to the wildtype background. This allows for the introduction of fresh genetic material and decreases the amount of inbreeding. Heterozygous ceb mice were created by crossing ceb knockout males with female C57BL/6 mice. Single knockouts for ceb were generated by crossing ceb heterozygous and knockout mice were identified by standard PCR genotyping. The second colony comprised mutant mice for synaptobrevin 2 all of which were ceb deficient. Double knockout mice were created by the crossing of syb 2 heterozygous and recovered by Cesarean section on embryonic day 18 (E18). Double knockout mice had a clearly discernable appearance with a tucked body shape (Borisovska et al., 2005). Their genotypes were verified by standard PCR genotyping: synaptobrevin 2 deficient mice were identified by PCR products essentially as described (Schoch et al., 2001). Cellubrevin mutant and wildtype alleles were identified by alternative PCR products. The reaction contained a mixture of a common forward primer (CAGACTCACTGAACCTATGAGAG) and two reverse primers for a mutant (CAGCGCATCGCCTTCTATCGC) and a wildtype (CTCACCTGATACATGCAGCAC) reaction. Cell cultures of adrenal chromaffin cells were prepared as previously described (Sorensen et al., 2003b), infected on days 2-4 after isolation and used 4-6 h after infection.

\subsection{Electrophysiology and electrochemistry}

Whole-cell patch clamp, membrane capacitance measurements, amperometry, ratiometric intracellular calcium $\left[\mathrm{Ca}^{2+}\right]_{\mathrm{i}}$ measurements and flash photolysis of 
caged $\mathrm{Ca}^{2+}$ were performed as described previously (Sorensen et al., 2006). Control and mutant constructs were expressed in cells from the same preparations in order to cancel variability between preparations. The displayed calcium concentrations, capacitance traces and amperometric currents are averaged over all cells recorded.

\subsubsection{Membrane capacitance as an assay of exocytosis}

The membrane of a cell in whole cell patch clamp configuration acts as a capacitor, since it is an electrical insulator separating two conducting compartments, namely the cytosol and the extracellular space (bath). The capacity of a capacitor is proportional to the area that can be charged, in this case the surface area of the cell. Measurements of cellular capacitance can thus be used to assay the increase of cellular surface area in exocytosis when vesicles fuse and the addition of vesicular membrane to the plasma membrane increases the capacitance (Neher and Marty, 1982).

In terms of an electrical equivalent circuit, the electrical properties of the cell are defined by a parallel combination of a capacitance across the plasma membrane and a resistance across the plasma membrane. Another resistance is added to this circuit by all practical means of electrical recordings: the series resistance of the recording electrode, which is in series to membrane resistance and membrane capacitance. A precise measurement of membrane capacitance can be performed in voltage clamp configuration by applying a sinusoidal signal and measuring the phase shift to the recorded current. With a pure parallel combination of membrane resistance and membrane capacitance without a resistance in series, the resulting current would be the sum of a component in phase (at the resistor: real part) with a component shifted in phase by $90^{\circ}$ (through the capacitor: imaginary part). Under all the conditions of a real experiment, the series resistance is non-zero and the relationship between input and output phase becomes more complex. Nonetheless, information on the properties of the circuit can still be extracted by use of hardware or software lock-in amplifiers. 
The capacitance measurements performed here are based on an admittance measurement. The admittance is the quotient of measured current and input voltage and likewise consists of a real part (the real part of the current divided by the voltage) and an imaginary part (the imaginary current divided by the voltage). The admittance analysis alone therefore only delivers two quantities. However, the circuit contains three unknowns: the membrane resistance, the membrane capacitance and the series resistance. In order to solve the problem and obtain the three unknowns, the Lindau-Neher Technique was used. It makes use of the application of an additional holding potential, summed with the sinusoidal voltage, allowing for the calculation of the three values (Sakmann and Neher, 1995).

Whole cell recordings were performed with sylgard coated and fire polished patch pipettes (Kimax-51; Kimble/Kontes, Vineland, NJ). An EPC-9 amplifier (HEKA Elektronik, Lamprecht/Pfalz, Germany) with the Pulse software (v 8.53) was used and membrane capacitance was assessed using the software lock-in feature. A $1 \mathrm{kHz}$ sinusoidal Voltage with a peak-to-peak amplitude of $70 \mathrm{mV}$ was superimposed on a DC holding potential of $-70 \mathrm{mV}$. Currents were filtered at $3 \mathrm{kHz}$ and sampled at $12 \mathrm{kHz}$.

\subsubsection{Electrochemistry detects neurotransmitter efflux}

Carbon fiber amperometry was performed as described previously (Sorensen et al., 2003b). Carbon fibers for the flash-experiments were $10 \mu \mathrm{m}$ in diameter (P100S; Amoco Corp., Greenvile, SC). Single spike analysis was performed with fibers of $5 \mu \mathrm{m}$ diameter, where noise was greatly reduced. Carbon fibers were attached with carbon paste to copper wires that could directly be attached to the amplifier headstage. The copper wire and carbon fiber were inserted into a glass capillary which was glued to the wire using epoxy glue. Glass capillaries were pulled on a pipette puller so that the carbon fiber extended through the pipette tip. The tip was then sealed with sylgard and the fiber was electrically insulated. A 
sensitive surface was obtained by cutting the fibre with a scalpel between recordings.

A constant voltage of $720 \mathrm{mV}$ was applied and fibers were pressed gently against the cell. Currents were amplified and filtered at $3 \mathrm{kHz}$ by an EPC-7 amplifier (HEKA Elektronik, Lamprecht/Pfalz, Germany). Upon loading into IGOR Pro version 6.04 (Wavemetrics, Lake Oswego, OR) currents were digitally filtered at $1 \mathrm{kHz}$ with a Gaussian filter and analyzed by a customized Macro. For single spike analysis, non-overlapping spikes with an amplitude larger than $10 \mathrm{pA}$ were considered.

Data for amperometric spike analysis were obtained in two independent experimental datasets, each comparing an $\mathrm{N}$ - and $\mathrm{C}$-terminal mutant and wildtype protein expressing cells from double knockout mice. Carbon fibers were frequently cut between recordings and systematically switched between all conditions tested. Statistics were compiled using the mean of cell medians of each parameter, because amperometric parameters generally display very large cell-tocell variability. Significance levels were assayed by analysis of Variance (ANOVA). The Effect of recording sequence, animal preparations and different fibers were tested by ANOVA but were all non-significant factors.

To identify standalone feet (SAF), two criteria were applied in order to distinguish SAF based on the shape of the signal. Following the characterization of Meyer Jackson and colleagues (Wang et al., 2006), SAF can be identified by their more rectangular shape. A quantitative readout of rectangularity may be the quotient of the times at which a signal crosses a characteristic value. Here two different criteria were used to analyze the data: $\mathrm{t} 1$ and $\mathrm{t} 2$ are two different duration measures, both starting at the point where the signal departs by more than 1xRMS (root-mean-square) of the noise above baseline. The end point of $\mathrm{t} 1$ was defined as the mean spike amplitude between the two time boundaries defined by the halfway points between peak amplitude and baseline. The end point of $\mathrm{t} 2$ is defined as the time when the signal returned to within 1xRMS of the baseline. Under the conditions of the current experiments these criteria did not seem ideal and therefore two new duration measures were defined: $t 3$ and $t 4$, whose end-points 
are identical to those of $\mathrm{t} 1$ and $\mathrm{t} 2$, but with the common starting point at the time the spike exceeds half its maximal amplitude. The latter criteria are independent of the foot duration, which in some cases caused problems in the assessment of SAF when applying the $\mathrm{t} 1 / \mathrm{t} 2$ criteria. For an illustration of these parameters please refer to Figure 22.

\subsubsection{Recording solutions}

The bath solution contained (in $\mathrm{mM}$ ): $145 \mathrm{NaCl}, 2.8 \mathrm{KCl}, 2 \mathrm{CaCl}_{2}, 1 \mathrm{MgCl}_{2}, 10$ HEPES, 11.1 glucose, adjusted to $\mathrm{pH} 7.2$ with $\mathrm{NaOH}$. The solution had an osmolarity of approximately $300 \mathrm{mOs}$.

The patch pipette solution contained (in $\mathrm{mM}$ ): 106 glutamic acid, $4 \mathrm{CaCl}_{2}, 35$ HEPES, 2 MgATP, 0.3 NaGTP, 5 nitrophenyl-EGTA, 0.5 ascorbic acid, 0.4 fura4f (Molecular Probes), 0.4 Magfura (Molecular Probes), adjusted to pH 7.2 with $\mathrm{CsOH}$. The solution had an osmolarity of approximately $300 \mathrm{mOs}$.

\subsection{4 $\mathrm{Ca}^{2+}$ photolysis and measurements of $\mathrm{Ca}^{2+}$ concentrations}

Photolysis of caged Calcium $\left(\mathrm{Ca}^{2+}\right)$ and intracellular measurements of $\mathrm{Ca}^{2+}$ concentrations were performed as described previously (Sorensen et al., 2002). $\mathrm{Ca}^{2+}$-uncaging was performed by application of a brief flash of UV light (Rapp Optoelektronik, Hamburg, Germany). Fluorescence excitation of $\mathrm{Ca}^{2+}$-sensitive dyes (see pipette solution) was achieved with monochromatic light (Polychrome IV monochromator; TILL Photonics, Planegg, Germany) at $350 \mathrm{~nm}$ and $380 \mathrm{~nm}$. In order to extend the range of $\mathrm{Ca}^{2+}$ measurements and allow for accurate measurement of $\mathrm{Ca}^{2+}$-concentrations from the nanomolar to the tens-ofmicromolar range, a mixture of the high affinity dye fura- $4 \mathrm{f}$ and the low affinity dye mag-fura was used (Schonn et al., 2008; Voets, 2000). Unlike the situation of experiments with a single dye, where fluorescence measurements in $\mathrm{Ca}^{2+}$-free and $\mathrm{Ca}^{2+}$ saturated states of the dye as well as a measurement at one known $\mathrm{Ca}^{2+}$ concentration suffice to determine the parameters of the Grynkiewicz-equation 
(Grynkiewicz et al., 1985), the measurements here require an in vivo calibration (Voets, 2000). For this purpose bovine chromaffin cells were patched with pipette solutions that contained the dye-mix and $\mathrm{Ca}^{2+}$ buffered to known concentrations. Measurement of fluorescence and fit of the $\mathrm{Ca}^{2+}$ dependency using a modified Grynkiewicz-equation allowed extraction of all parameters necessary to accurately measure $\mathrm{Ca}^{2+}$ levels.

\subsubsection{Kinetic analysis of capacitance responses}

For statistical analysis, it was in some cases distinguished between the first second of the capacitance increase following stimulation (burst component), and the capacitance increase taking place during the following four seconds (sustained component). In wild type cells, these two components are often of similar size, but the burst component mainly assays the fusion of vesicles that were pre-primed before photolysis, whereas the sustained component assays vesicles that had to undergo one or more priming steps before fusing. In other cases, detailed kinetic analysis was employed, essentially as described previously (Sorensen et al., $2003 b$ ). For this a triple exponential function was fit to individual capacitance traces (Equation 1).

(1) $\quad f(t)=A_{0}+\sum_{i=0}^{3} A_{i}\left(1-e^{-\frac{\left(t-t_{0}\right)}{\tau_{i}}}\right)$

Where $A_{0}$ is the capacitance of the cell before stimulation and $t_{0}$ is the time of the flash. The fastest exponential defines the size and time constant of the readily releasable pool (RRP): $\mathrm{A}_{1}$ reflects the size of the RRP and $\tau_{1}$ is $\tau_{\mathrm{RRP}}$. Likewise $\mathrm{A}_{2}$ is the size of the slowly releasable pool (SRP) and $\tau_{2}$ is $\tau_{\text {SRP. }}$. The third exponential term would correspond to the sustained pool. Since the time constant of $\tau_{3}$ is typically very large, this behavior on the time scale of the experiment is almost linear. Following this notion, a sustained time constant was not analyzed and the sustained component was measured as the change in capacitance from 1 second to 5 seconds assuming linear behavior. To obtain the sustained rate this value is 
divided by four seconds. An example of the fit of above function to capacitance data as well as an illustration of the different parameters can be found in Figure 4.

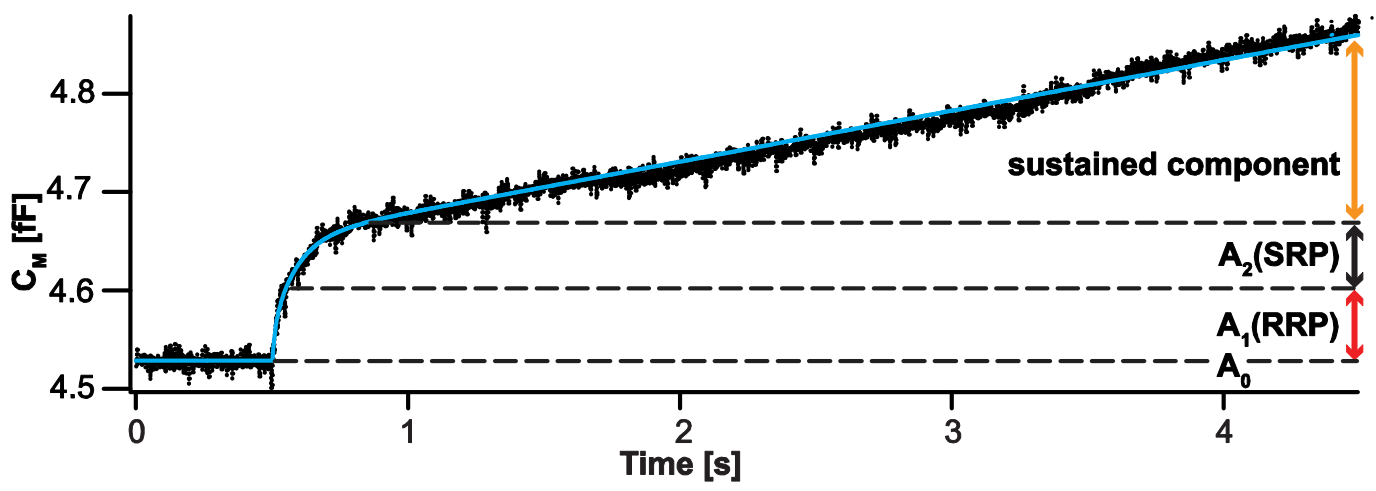

Figure 4. An example for the kinetic analysis of capacitance traces and an illustration of pool parameters. A typical response of a mouse chromaffin cell in flash-evoked release: the cellular capacitance increase follows a multi-exponential behavior which can be attributed to the release of different kinetic pools. The parameters obtained by the fit of Equation 1 to the data (blue trace) allow for the calculation of cell capacitance prior to release $\left(A_{0}\right)$, two amplitudes of exponential increase $\left(A_{1}\right.$ and $\left.A_{2}\right)$, their time course $\left(\tau_{1}\right.$ and $\tau_{2}$, not shown) and the sustained component.

Data are presented as mean \pm SEM. Statistical analysis was performed using Utest or t-tests on homoscedastic data, assessed by Levene's test of equality of variance. Where necessary, hetereoscedastic data was log-transformed to yield homoscedastic data before testing.

\subsection{Immunocytochemistry}

Embryonic syb/ceb double knockout and wild type chromaffin cells were cultured on poly-L-lysine coated coverslips. Cells infected with syb 2 or syt 1 constructs with or without the mutation as well as non-infected controls were fixed in $3.7 \%$ para-formaldehyde (PFA) in PBS for $20 \mathrm{~min}$, washed and permeablilized in $0.2 \%$ Triton X-100. Remaining PFA was neutralized in $50 \mathrm{mM} \mathrm{NH} 4 \mathrm{Cl}$. Cells were blocked in $2 \%$ bovine albumin serum (Sigma) and incubated for $2 \mathrm{~h}$ with primary antibodies [(1) mouse anti-syb 2, dilution 1:500 and rabbit anti-syt 1, dilution 1:200, both courtesy of Reinhard Jahn, Max-Planck-Institute for Biophysical Chemistry, Göttingen, Germany. (2) mouse anti-synaptotagmin-1, dilution 1:100 
(Synaptic Systems, Göttingen, Germany) and rabbit anti-Chromogranin A, dilution 1:500 (abcam). As well as (3) mouse anti-syb 2, dilution 1:500 and rabbit anti-Chromogranin A dilution 1:500.]. Cells were washed four times, incubated with secondary antibodies (Alexa546-conjugated goat anti-mouse, dilution 1:200 and Alexa647-conjugated goat anti-rabbit, dilution 1:200), washed and imaged. Fluorescence quantification was performed on a Zeiss Axiovert 200 microscope, fluorescence excitation was achieved by monochromatic light (Polychrome V, TILL Photonics) and images were analyzed using TILLvisION (TILL Photonics, v4.0.1). Fluorescence levels were quantified as the integrated intensity of a square region of the image containing the cell minus the intensity of a background region of the same size. Data were averaged over cells and are represented as mean and SEM. Confocal imaging was performed on a Leica TCS SP5 microscope, image processing was employed using LAS AF software (Leica, v1.7.0 build 1111).

\subsection{Delineation of the Meyer Jackson model for pool kinetics}

Much of the work presented here deals with the characterization of single fusion events studied by means of carbon fiber amperometry. The characterization of the intermediate state of the fusion pore is of particular interest. As mentioned previously, the molecular nature of the pore is under debate (Jackson and Chapman, 2006). Although not the main scope of this thesis, the implications of the proteinaceous pore model which suggests ion-channel-like behavior with reversible opening will be addressed. If such a model were true, it would be expected that the extension of the model to vesicular pools should be able to predict the behavior of capacitance responses in chromaffin cells. Particularly the multi-exponential property of capacitance responses would have to be represented in such a model in order to comply with experimental data. As shall be shown, the Meyer Jackson model cannot account for these observations.

According to Meyer Jackson and colleagues (Wang et al., 2001), the behavior of the fusion pore can be described by the following kinetic scheme: 
(2) $\quad P \underset{k_{c}}{\stackrel{k_{o}}{\rightleftarrows}} O \stackrel{k_{f}}{\rightarrow} F$

Here, $\mathrm{P}$ describes the state of the primed, releasable vesicle. Transition to the open state $(\mathrm{O})$ occurs with a rate constant of $\mathrm{k}_{\mathrm{o}}$. From the open state, two transitions are possible: pore re-closure with a rate constant of $\mathrm{k}_{\mathrm{c}}$, or, irreversible relaxation of the pore to the fused $(\mathrm{F})$ state with a rate constant $\mathrm{k}_{\mathrm{f}}$.

(3) $\frac{d P}{d t}=-k_{o} P+k_{c} O$

(4) $\frac{d O}{d t}=k_{o} P-\left(k_{c}+k_{f}\right) O$

In Matrix form, above equations can be written as follows:

(5) $\quad \frac{d}{d t}\left(\begin{array}{l}P \\ O\end{array}\right)=\boldsymbol{K}\left(\begin{array}{l}P \\ O\end{array}\right)$

(6) $\quad \boldsymbol{K}=\left(\begin{array}{cc}-k_{o} & k_{c} \\ k_{o} & -k_{c}-k_{f}\end{array}\right)$

$$
\frac{d}{d t}\left(\begin{array}{l}
P \\
O
\end{array}\right)=\left(\begin{array}{cc}
-k_{o} & k_{c} \\
k_{o} & -k_{c}-k_{f}
\end{array}\right)\left(\begin{array}{l}
P \\
O
\end{array}\right)
$$

Determination of the eigenvalues yields:

$$
\lambda_{a}=-\frac{k_{o}+k_{c}+k_{f}}{2}+\sqrt{\frac{\left(k_{o}+k_{c}+k_{f}\right)^{2}}{4}-k_{o} k_{f}}
$$

(9) $\quad \lambda_{b}=-\frac{k_{o}+k_{c}+k_{f}}{2}-\sqrt{\frac{\left(k_{o}+k_{c}+k_{f}\right)^{2}}{4}-k_{o} k_{f}}$

For $\mathrm{k}_{\mathrm{o}}, \mathrm{k}_{\mathrm{c}}$ and $\mathrm{k}_{\mathrm{f}}>0$ the eigenvalues are real and different.

Calculation of the eigenvector matrix (B) using the following equation

$$
\text { (10) }(\mathbf{K}-\lambda \mathbf{E}) \boldsymbol{x}=\mathbf{0}
$$

in which $\mathbf{E}$ is the unit matrix and $\mathbf{0}$ is the zero vector, yields:

$$
\text { (11) } \boldsymbol{B}=\left(\begin{array}{ll}
x_{1}^{1} & x_{1}^{2} \\
x_{2}^{1} & x_{2}^{2}
\end{array}\right)
$$


Where $\mathbf{x}^{1}$ and $\mathbf{x}^{2}$ are the eigenvectors for $\lambda_{\mathrm{a}}$ and $\lambda_{\mathrm{b}}$, respectively.

(12) $\quad \boldsymbol{B}=\left(\begin{array}{cc}C_{1} & C_{2} \\ \frac{k_{o}+\lambda_{a}}{k_{c}} C_{1} & \frac{k_{o}+\lambda_{b}}{k_{c}} C_{2}\end{array}\right)$

$\mathrm{C}_{1}$ and $\mathrm{C}_{2}$ are integration constants. The general solution of the model is thus given by:

(13) $P(t)=C_{1} e^{\lambda_{a} t}+C_{2} e^{\lambda_{b} t}$

(14) $O(t)=\frac{k_{o}+\lambda_{a}}{k_{c}} C_{1} e^{\lambda_{a} t}+\frac{k_{o}+\lambda_{b}}{k_{c}} C_{2} e^{\lambda_{b} t}$

The values of $\mathrm{C}_{1}$ and $\mathrm{C}_{2}$ can be determined by the initial conditions in the uncaging experiments. At time zero, prior to stimulation, the number of unfused vesicles is $\mathrm{P}(0)=\mathrm{P}_{0}$ and no vesicles are in the open state or fused state: $\mathrm{O}(0)=0$, $F(0)=0$. Inserting these initial values in the general solution one obtains:

(15) $C_{1}=\frac{k_{o}+\lambda_{b}}{\lambda_{b}-\lambda_{a}} P_{0}$

(16) $C_{2}=\frac{k_{o}+\lambda_{a}}{\lambda_{a}-\lambda_{b}} P_{0}$

Inserting these values in $(12,13)$ one obtains the specific solution:

$$
\begin{aligned}
& P(t)=\frac{k_{o}+\lambda_{b}}{\lambda_{b}-\lambda_{a}} P_{0} e^{\lambda_{a} t}+\frac{k_{o}+\lambda_{a}}{\lambda_{a}-\lambda_{b}} P_{0} e^{\lambda_{b} t} \\
& O(t)=\frac{k_{o}+\lambda_{a}}{k_{c}} \frac{k_{o}+\lambda_{b}}{\lambda_{b}-\lambda_{a}} P_{0} e^{\lambda_{a} t}+\frac{k_{o}+\lambda_{b}}{k_{c}} \frac{k_{o}+\lambda_{a}}{\lambda_{a}-\lambda_{b}} P_{0} e^{\lambda_{b} t}
\end{aligned}
$$

By mass conservation (at all times the total number of vesicles must amount to $\left.\mathrm{P}_{0}\right)$ :

$$
F(t)=P_{0}-O(t)-P(t)
$$

Both fully fused and vesicles in the open state contribute the cell's membrane capacitance $\mathrm{C}_{\mathrm{M}}$. Hence:

$$
C_{M}=C_{0}+O(t)+F(t)
$$




$$
C_{M}=C_{0}+P_{0}-P(t)
$$

And, after subtraction of the basal capacitance $\mathrm{C}_{0}$ :

$$
\text { (22) } \Delta C_{M}=C_{M}-C_{0}=P_{0}-P(t)
$$

Finally, the membrane capacitance in terms of poolsize $\left(\mathrm{P}_{0}\right)$ and rate constants is given as:

$$
\Delta C_{M}=P_{0}\left(1-\frac{k_{o}+\lambda_{b}}{\lambda_{b}-\lambda_{a}} e^{\lambda_{a} t}-\frac{k_{o}+\lambda_{a}}{\lambda_{a}-\lambda_{b}} e^{\lambda_{b} t}\right)
$$

\subsubsection{The fusion pore forms essentially irreversibly}

It can be shown that according to the model, the capacitance trace $\left(\Delta \mathrm{C}_{\mathrm{M}}\right)$ should contain a component with a time constant faster than the mean lifetime of the fusion pore $\tau_{\mathrm{o}}$.

Proposition 1: The mean lifetime of the fusion pore with respect to the above scheme (Equation 2) is given as follows:

$$
\text { (24) } \tau_{o}=\frac{1}{k_{f}+k_{c}}
$$

It shall be shown that the fastest rate constant of the double exponential function (Equation 23), which is given by $\lambda_{\mathrm{b}}$, is smaller than the inverse mean foot duration $\tau_{\mathrm{o}}$ (Equation 24).

$$
\begin{aligned}
& \text { (25) }-\lambda_{b} \geq \frac{1}{\tau_{O}} \\
& -2 \lambda_{b}=\left(k_{o}+k_{c}+k_{f}\right)+\sqrt{\left(k_{o}+k_{c}+k_{f}\right)^{2}-4 k_{o} k_{f}} \\
& -2 \lambda_{b}=\left(k_{o}+k_{c}+k_{f}\right)+\sqrt{k_{o}^{2}+k_{c}^{2}+k_{f}^{2}+2 k_{o} k_{c}-2 k_{o} k_{f}+2 k_{c} k_{f}} \\
& -2 \lambda_{b} \geq\left(k_{c}+k_{f}\right)+\sqrt{k_{o}^{2}+k_{c}^{2}+k_{f}^{2}-2 k_{o} k_{c}-2 k_{o} k_{f}+2 k_{c} k_{f}}
\end{aligned}
$$




$$
\begin{aligned}
& -2 \lambda_{b} \geq 2 k_{c}+2 k_{f} \\
& -2 \lambda_{b} \geq \frac{2}{\tau_{o}}
\end{aligned}
$$

Above calculations infer that the capacitance response should contain a component with a time constant at least as fast as the mean pre-spike foot duration, which under our conditions is roughly in the range of $5 \mathrm{~ms}$ (Figure 20). However, such a component is normally not found in capacitance recordings (Figures 14, 18). In order to comply with the behavior typically observed in capacitance measurements, the amplitude of this component should therefore be zero. It can be shown, however, that this condition is only met, if $\mathrm{k}_{\mathrm{c}}$ is essentially zero:

Proposition 2: The behavior of the capacitance response can only be met if $\mathrm{k}_{\mathrm{c}}$ is zero.

The component, whose speed is determined by $\lambda_{b}$, has the following amplitude (Equation 23):

$$
\text { (27) }-\frac{k_{o}+\lambda_{a}}{\lambda_{a}-\lambda_{b}}
$$

In order for this amplitude to vanish, $\mathrm{k}_{\mathrm{o}}$ should be equal to $-\lambda_{\mathrm{a}}$.

$$
\text { (28) } k_{o}=-\lambda_{a}
$$

Therefore:

$$
k_{o}=-\lambda_{a}=\frac{k_{o}+k_{c}+k_{f}}{2}-\frac{\sqrt{\left(k_{o}+k_{c}+k_{f}\right)^{2}-4 k_{o} k_{f}}}{2}
$$

Rearranging for $\mathrm{k}_{\mathrm{f}}$ gives the following expression:

$$
\begin{aligned}
& k_{f}=k_{o}-k_{c}+\sqrt{\left(k_{o}+k_{c}+k_{f}\right)^{2}-4 k_{o} k_{f}} \\
& k_{f}=k_{o}-k_{c}+\sqrt{k_{o}^{2}+k_{c}^{2}+k_{f}^{2}+2 k_{o} k_{c}+2 k_{o} k_{f}+2 k_{c} k_{f}-4 k_{o} k_{f}}
\end{aligned}
$$




$$
\begin{aligned}
& k_{f}=k_{o}-k_{c}+\sqrt{k_{o}^{2}+k_{c}^{2}+k_{f}^{2}+2 k_{o} k_{c}-2 k_{o} k_{f}+2 k_{c} k_{f}} \\
& k_{f} \geq k_{o}-k_{c}+\sqrt{k_{o}^{2}+k_{c}^{2}+k_{f}^{2}-2 k_{o} k_{c}-2 k_{o} k_{f}+2 k_{c} k_{f}} \\
& k_{f} \geq k_{o}-k_{c}+\sqrt{\left(-k_{o}+k_{c}+k_{f}\right)^{2}} \\
& k_{f} \geq k_{o}-k_{c}-k_{o}+k_{c}+k_{f} \\
& k_{f} \geq k_{f}
\end{aligned}
$$

This implies that

$$
\text { (31) } k_{o} k_{c}=0
$$

And, for the non-trivial case of $k_{0}>0$ requires that $k_{c}$ be zero $\left(k_{c}=0\right)$.

Still, in theory one can imagine that such a fast component may exist, but remains undetected due to the fact that its amplitude does not exceed the noise level of the measurement. In the light of noise typically observed in such recordings this implies that such a component cannot have an amplitude exceeding $3 \mathrm{fF}$. Equation 27 should thus amount to less than $3 \mathrm{fF}$. With a typical size of $70 \mathrm{fF}$ for the RRP, this corresponds to a relative amplitude of $4 \%$ for the fast component (Equation 32). With typical values for the other parameters one can estimate the relative frequency of standalone feet. Considering Equation 24 and an average foot duration of $5 \mathrm{~ms}$ and a typical time constant of the RRP of approximately $20 \mathrm{~ms}$, one can estimate:

$$
\begin{aligned}
& -\frac{k_{o}+\lambda_{a}}{\lambda_{a}-\lambda_{b}}=\frac{3}{70}=4 \% \\
& \lambda_{b} \approx-\frac{1}{\tau_{o}}=-\frac{1}{5 \mathrm{~ms}}=-200 \mathrm{~s}^{-1} \\
& \lambda_{a}=-\frac{1}{\tau_{R R P}}=-\frac{1}{20 \mathrm{~ms}}=-50 \mathrm{~s}^{-1}
\end{aligned}
$$

From Equation 32, $\mathrm{k}_{\mathrm{o}}$ can be calculated:

$$
k_{o}=0.04 *\left(\lambda_{a}-\lambda_{b}\right)-\lambda_{a}=56 s^{-1}
$$

According to Equation 8, 9: 
(36) $-\lambda_{a}-\lambda_{b}=k_{o}+k_{c}+k_{f}$

(37) $k_{c}=-\lambda_{a}-\lambda_{b}-k_{o}-k_{f}$

And:

(38) $k_{o} k_{f}=\lambda_{a} \lambda_{b}=10000 \mathrm{~s}^{-2}$

Therefore:

(39) $k_{f}=180 \mathrm{~s}^{-1}$

(40) $k_{c}=15 \mathrm{~s}^{-1}$

The relative occurrence of standalone feet can now be calculated:

(41) $P(S A F)=\frac{\mathrm{k}_{\mathrm{c}}}{\mathrm{k}_{\mathrm{c}}+\mathrm{k}_{\mathrm{f}}}<8 \%$

Under these assumptions, standalone feet should occur in less than $8 \%$ of all secretion events. This value represents an upper estimate. 


\subsection{Derivation of Yet Even Another Hypothesis ("YEAH")}

Although conceptually attractive the above model fails to describe pool kinetics. This is unfortunate since a direct comparison of effects on single spikes and pool kinetics would be of particular interest. Most researchers consider these experiments in separation and conclusions from experiments on single spikes cannot be used to make predictions regarding the behavior of kinetic pools in the flash experiment. An elegant feature of the model proposed by Meyer Jackson and its extension to pool kinetics is that the reactions are defined by elementary molecular reactions described in a chemical equation.

This is different from the application of the Voets parallel pool model, which by empirical inspection of capacitance traces proposes the existence of separate pools, independent in their size and speed of secretion. The existence of separate pools is thought to arise from heterogeneity on a molecular level (Voets et al., 1999), but some assumptions of this model are hard to grasp. For instance, it remains to be established how a vesicle can be discerned as to whether it is part of a rapid or a slow pool. The observations in the flash experiment could be explained by the action of different $\mathrm{Ca}^{2+}$ sensors. This is, of course, a reasonable explanation, but alternative hypotheses may also explain this. For instance, a maturation of the vesicle in order to gain release competence may well involve a pre-equilibrium. The implications of a pre-equilibrium to a releasable state for the time course of secretion will be dealt with in due course.

Together, the Voets model allows excellent empirical description of the data in mathematical terms. However, it lacks insight into the fundamental processes and reactions involved in the formation of the pools as well as an understanding of how pool size and release rate are coupled. The Meyer Jackson model is elegant in the sense that it is merely based on elementary reactions of a very simple kinetic scheme. An experimental observation could thus be used to characterize individual chemical reactions. However, this model fails in the description of the flash-data, suggesting that some features of the model are inadequate. 
In the light of these considerations, a new model can be developed, starting from a chemical equation describing the maturation and release process of secretory vesicles. Above calculations establish that pore re-closure under the conditions of the flash-experiment is a rare event. A model with a pre-equilibrium but irreversible fusion pore formation may well be utilized to model pool kinetics. For this, a number of assumptions are invested:

1 A pre-equilibrium exists between non-releasable (NR) vesicles and releasable (R) vesicles.

2 Opening the fusion pore is an irreversible process.

3 Detection of a capacitance increase in the open $(\mathrm{O})$ and fused $(\mathrm{F})$ state. The capacitance measurement is therefore "blind" to a transition of O to F.

A model with above features can be described by the following chemical and sets of kinetic equations:

$$
\begin{aligned}
& \text { (42) } N R \underset{k_{-1}}{\stackrel{k_{1}}{\rightleftarrows}} R \stackrel{k_{2}}{\rightarrow} O \stackrel{k_{3}}{\rightarrow} F \\
& \text { (43) } \frac{d N R}{d t}=-k_{1} N R+k_{-1} R \\
& \text { (44) } \frac{d R}{d t}=k_{1} N R-\left(k_{-1}+k_{2}\right) R \\
& \text { (45) } \frac{d O}{d t}=k_{2} R-k_{3} O \\
& \text { (46) } \frac{d F}{d t}=k_{3} O
\end{aligned}
$$

The capacitance increase is not dependent on a further reaction from the $\mathrm{O}$ to the $\mathrm{F}$ state, since both $\mathrm{O}$ and $\mathrm{F}$ vesicles contribute to the cell's membrane capacitance $\mathrm{C}_{\mathrm{M}}$ and are indistinguishable in the capacitance measurement. Thus, Equation 46 is not necessary for the description of the capacitance course and the second term in Equation 45 can be neglected. The kinetic equations can be rewritten in matrix form: 
(47) $\frac{d}{d t}\left(\begin{array}{c}N R \\ R \\ O\end{array}\right)=\left(\begin{array}{ccc}-k_{1} & k_{-1} & 0 \\ k_{1} & -k_{-1}-k_{2} & 0 \\ 0 & k_{2} & 0\end{array}\right)\left(\begin{array}{c}N R \\ R \\ O\end{array}\right)$

Determination of the eigenvalues yields:

(48) $\lambda_{1}=-\frac{k_{1}+k_{-1}+k_{2}}{2}+\sqrt{\frac{\left(k_{1}+k_{-1}+k_{2}\right)^{2}}{4}-k_{1} k_{2}}$

(49) $\lambda_{2}=-\frac{k_{1}+k_{-1}+k_{2}}{2}-\sqrt{\frac{\left(k_{1}+k_{-1}+k_{2}\right)^{2}}{4}-k_{1} k_{2}}$

(50) $\lambda_{3}=0$

The eigenvector matrix is given according to Equation 10:

(51) $\quad \boldsymbol{B}=\left(\begin{array}{ccc}a_{1} & a_{2} & 0 \\ a_{1} \frac{k_{1}+\lambda_{1}}{k_{-1}} & a_{2} \frac{k_{1}+\lambda_{2}}{k_{-1}} & 0 \\ a_{1} \frac{k_{2}}{\lambda_{1} \frac{k_{1}+\lambda_{1}}{k_{-1}}} & a_{2} \frac{k_{2}}{\lambda_{2} \frac{k_{1}+\lambda_{2}}{k_{-1}}} & a_{3}\end{array}\right)$

The general solution is:

(52) $\left(\begin{array}{c}N R \\ R \\ O\end{array}\right)=\left(\begin{array}{ccc}a_{1} & a_{2} & 0 \\ a_{1} \frac{k_{1}+\lambda_{1}}{k_{-1}} & a_{2} \frac{k_{1}+\lambda_{2}}{k_{-1}} & 0 \\ a_{1} \frac{k_{2}}{\lambda_{1} \frac{k_{1}+\lambda_{1}}{k_{-1}}} & a_{2} \frac{k_{2}}{\lambda_{2} \frac{k_{1}+\lambda_{2}}{k_{-1}}} & a_{3}\end{array}\right)\left(\begin{array}{l}C_{1} e^{\lambda_{1} t} \\ C_{2} e^{\lambda_{2} t} \\ C_{3} e^{\lambda_{3} t}\end{array}\right)$

Under consideration of $\lambda_{3}=0$ and combining the integration constants $C_{x}$ and $a_{x}$, which are each arbitrary constants:

$$
\text { (53) } \begin{aligned}
& a_{1} C_{1}=\tilde{C}_{1} \\
& a_{2} C_{2}=\tilde{C}_{2} \\
& a_{3} C_{3}=\tilde{C}_{3}
\end{aligned}
$$

The individual solutions are:

$$
\begin{aligned}
& \text { (54) } N R(t)=\tilde{C}_{1} e^{\lambda_{1} t}+\tilde{C}_{2} e^{\lambda_{2} t} \\
& \text { (55) } \quad R(t)=\frac{k_{1}+\lambda_{1}}{k_{-1}} \tilde{C}_{1} e^{\lambda_{1} t}+\frac{k_{1}+\lambda_{2}}{k_{-1}} \tilde{C}_{2} e^{\lambda_{2} t}
\end{aligned}
$$


(56)

$$
O(t)=\frac{k_{2}}{\lambda_{1}} \frac{k_{1}+\lambda_{1}}{k_{-1}} \tilde{C}_{1} e^{\lambda_{1} t}+\frac{k_{2}}{\lambda_{2}} \frac{k_{1}+\lambda_{2}}{k_{-1}} \tilde{C}_{2} e^{\lambda_{2} t}+\tilde{C}_{3}
$$

The initial conditions for the determination of the integration constants are that prior to stimulation no vesicles are in the open state $(\mathrm{O})\left(\mathrm{k}_{2}=0\right)$ and the total number of vesicles $\left(\mathrm{V}_{\text {tot }}\right)$ distributed in $\mathrm{NR}$ and $\mathrm{R}$ are given by the following equations:

(57) $\quad N R(0)=V_{\text {tot }} \frac{k_{-1}}{k_{1}+k_{-1}}$

$$
R(0)=V_{t o t} \frac{k_{1}}{k_{1}+k_{-1}}
$$

$$
O(0)=0
$$

The values of the integration constants are given by:

(60) $\quad \tilde{C}_{1}=V_{t o t} \frac{k_{-1} \lambda_{2}}{\left(\lambda_{2}-\lambda_{1}\right)\left(k_{1}+k_{-1}\right)}$

(61) $\quad \tilde{C}_{2}=V_{t o t} \frac{k_{-1} \lambda_{1}}{\left(\lambda_{1}-\lambda_{2}\right)\left(k_{1}+k_{-1}\right)}$

(62) $\tilde{C}_{3}=V_{\text {tot }}$

The cellular capacitance change is given by the change in the number of vesicles in the open state:

(63) $\Delta C_{M}=O(t)$

(64) $\Delta C_{M}=V_{\text {tot }}\left(1-\frac{\lambda_{2}}{\lambda_{1}} \frac{k_{2}\left(k_{1}+\lambda_{1}\right)}{\left(\lambda_{2}-\lambda_{1}\right)\left(k_{1}+k_{-1}\right)} e^{\lambda_{1} t}-\frac{\lambda_{1}}{\lambda_{2}} \frac{k_{2}\left(k_{1}+\lambda_{2}\right)}{\left(\lambda_{1}-\lambda_{2}\right)\left(k_{1}+k_{-1}\right)} e^{\lambda_{2} t}\right)$

This equation was used to fit the capacitance response of the burst phase of secretion using Origin 8 SR2 (v8.0891). 


\section{Results}

\subsection{The SNAREs in docking}

The attachment of secretory vesicles to their release site is an essential prerequisite for vesicles to undergo fusion. Docking, the stable attachment of vesicles to their release site is implicated as a precondition for the downstream processes of vesicle maturation and fusion. Two major docking factors have been identified based on their necessity to promote docking in chromaffin cells and neurons: Munc-18 and syntaxin 1 (de Wit et al., 2006; Hammarlund et al., 2007; Voets et al., 2001b; Weimer et al., 2003). These proteins have also been shown to be involved in important downstream reactions of the vesicle's release cycle: Munc-18 by its role in vesicle priming (Deak et al., 2009; Gulyas-Kovacs et al., 2007) and syntaxin, as a protein of the SNARE complex in triggered exocytosis (Gerber et al., 2008). Syntaxin is a plasma membrane-bound protein, localized at the target site, whereas Munc-18 is a soluble protein. Munc-18 binds syntaxin in its "closed" conformation (Dulubova et al., 1999). For rapid liposome fusion, formation of a 1:1 SNAP-25:syntaxin acceptor complex is necessary (Pobbati et al., 2006). So far it has not been established whether this also constitutes a necessity for docking in vivo. In order to test whether a syntaxin:SNAP-25 acceptor complex is an essential docking platform for subsequent attachment of the vesicle to the target membrane, morphological and electrophysiological experiments in a number of mutant mice using rescue and cross-rescue approaches were employed.

Docking was investigated by ultrastructural analysis of cell morphology in the different conditions by measuring the distance between vesicle and plasma membrane. Docking becomes apparent as a skew of the distribution towards shorter distances. A defect in docking therefore becomes apparent when fewer vesicles are found close to the plasma membrane. The number of vesicles docked was determined as the number of vesicles without measurable distance to the plasma membrane. 
In order to characterize exocytosis physiologically, whole cell capacitance measurements in adrenal chromaffin cells were employed (Neher, 2006). Here, exocytosis can be triggered by photolysis of caged $\mathrm{Ca}^{2+}$, leading to the release of primed vesicles. Fused vesicles add to the cellular membrane capacitance due to the addition of vesicular membrane to the plasma membrane. Hence, fusion can be monitored by cellular capacitance measurements (Lindau and Neher, 1988). Moreover, transmitter release from dense core vesicles can be detected by carbon fiber amperometry (Wightman et al., 1991). Initial capacitance increase in response to flash-evoked release by previously primed vesicles is followed by sustained secretion, due to the experimental conditions in which $\mathrm{Ca}^{2+}$ concentrations remain high and newly primed vesicles undergo fusion. In these conditions the priming rate becomes rate limiting.

\subsubsection{SNAP-25 is an essential docking factor}

Under the assumption that syntaxin:SNAP-25 interaction is necessary for docking, it should be expected that docking is impaired in the absence of either proteins. This is established for syntaxin (de Wit et al., 2006). However, similar effects for SNAP-25 had not been reported and previous analysis of docking in cells deficient of SNAP-25 had not revealed a docking defect (Sorensen et al., 2003b). Detailed re-analysis of these mice, however, did show a strong defect in vesicle docking in SNAP-25 nulls. This defect was reproducibly shown in preparations using whole fixed glands and dissociated cells in culture as well as tissue conserved by high pressure rapid freezing (de Wit et al., 2009). Further evidence towards the specificity of this effect was given by the successful rescue of the docking defect after overexpression of SNAP-25 in KO cells.

\subsubsection{Munc-18 promotes docking by stabilizing a syntaxin:SNAP-25 acceptor complex}

As previously reported, absence of Munc-18 in chromaffin cells produces a severe defect in vesicle docking (Voets et al., 2001b). In order to investigate the role of 
Munc-18 in docking and a possible interplay with SNAP-25, a cross-rescue approach was followed. Overexpression of SNAP-25 in cells deficient of Munc18 could restore docking, but was insufficient to rescue secretion (de Wit et al., 2009). This suggests an essential interplay of Munc-18 and SNAP-25 for the generation of a functional docking platform at the plasma membrane. Munc-18 may act by stabilizing a syntaxin:SNAP-25 acceptor complex. This stabilization may consider the stoichometry. It is known from experiments in-vitro, that syntaxin and SNAP-25 can form stable 2:1 complexes, where a single molecule of SNAP-25 attaches to two molecules of syntaxin (Fasshauer and Margittai, 2004). In-vitro formation of this complex interferes with synaptobrevin binding and SNARE-mediated liposome fusion (Pobbati et al., 2006). This complex may, however, also play a role in docking where Munc-18 may act by stabilizing the 1:1 syntaxin:SNAP-25 complex. In the absence of Munc-18, overexpression of SNAP-25 may facilitate docking by shifting the equilibrium toward higher concentrations of the 1:1 complex by the law of mass action.

If indeed Munc-18 serves as a factor for stabilizing a 1:1 syntaxin:SNAP-25 acceptor complex, it would be expected that stabilization of such a complex by an alternative strategy may succeed in rescuing the docking defect in Munc-18 deficient cells. A similar approach of this sort has previously been employed in invitro studies (Pobbati et al., 2006), where the addition of a soluble fragment of synaptobrevin prevented formation of an unproductive 2:1 synatxin:SNAP-25 acceptor complex (off-pathway) (Fasshauer and Margittai, 2004). Only C-terminal fragments but not N-terminally bound fragments were able to produce the speedup of SNARE complex assembly, consistent with the idea, that initial binding of synaptobrevin to SNARE partners is mediated by its N-terminus. Utilizing soluble fragments of synaptobrevin, it was tested whether docking could be restored in Munc-18 deficient cells. Indeed, expression of a C- but not N-terminal syb fragment sufficed to restore docking to control levels (Figure 5A, D). Interestingly, rescue of docking in these cells was not accompanied by functional rescue of secretion (Figure 5E, F): unlike the condition of Munc-18 (M18) expression, where a robust increase in cellular capacitance $\left(\Delta C_{M}\right)$ was observed in 
response to stimulation (at $\mathrm{t}=0.5 \mathrm{~s}$ ), cells expressing $\mathrm{C}$ - and $\mathrm{N}$-terminal syb fragments behaved like Munc-18 knockout (KO) cells. This suggests an important role of Munc-18 downstream of docking consistent with the supposed role of Munc-18 in vesicle priming (Deak et al., 2009; Gulyas-Kovacs et al., 2007). However, $\mathrm{N}$ - or $\mathrm{C}$-terminal fragments of synaptobrevin also failed to influence secretion in the presence of Munc-18, since expression of neither $\mathrm{N}$ - nor Cterminal syb fragments in wildtype cells showed an effect on secretion (Figure 12B).
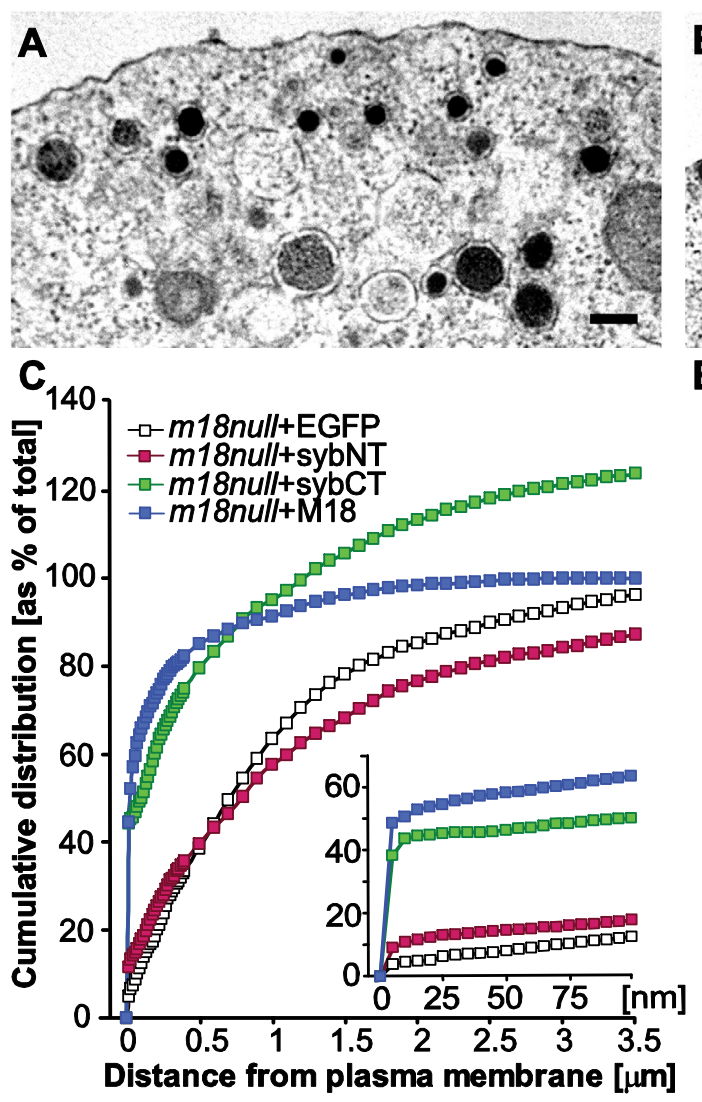

B
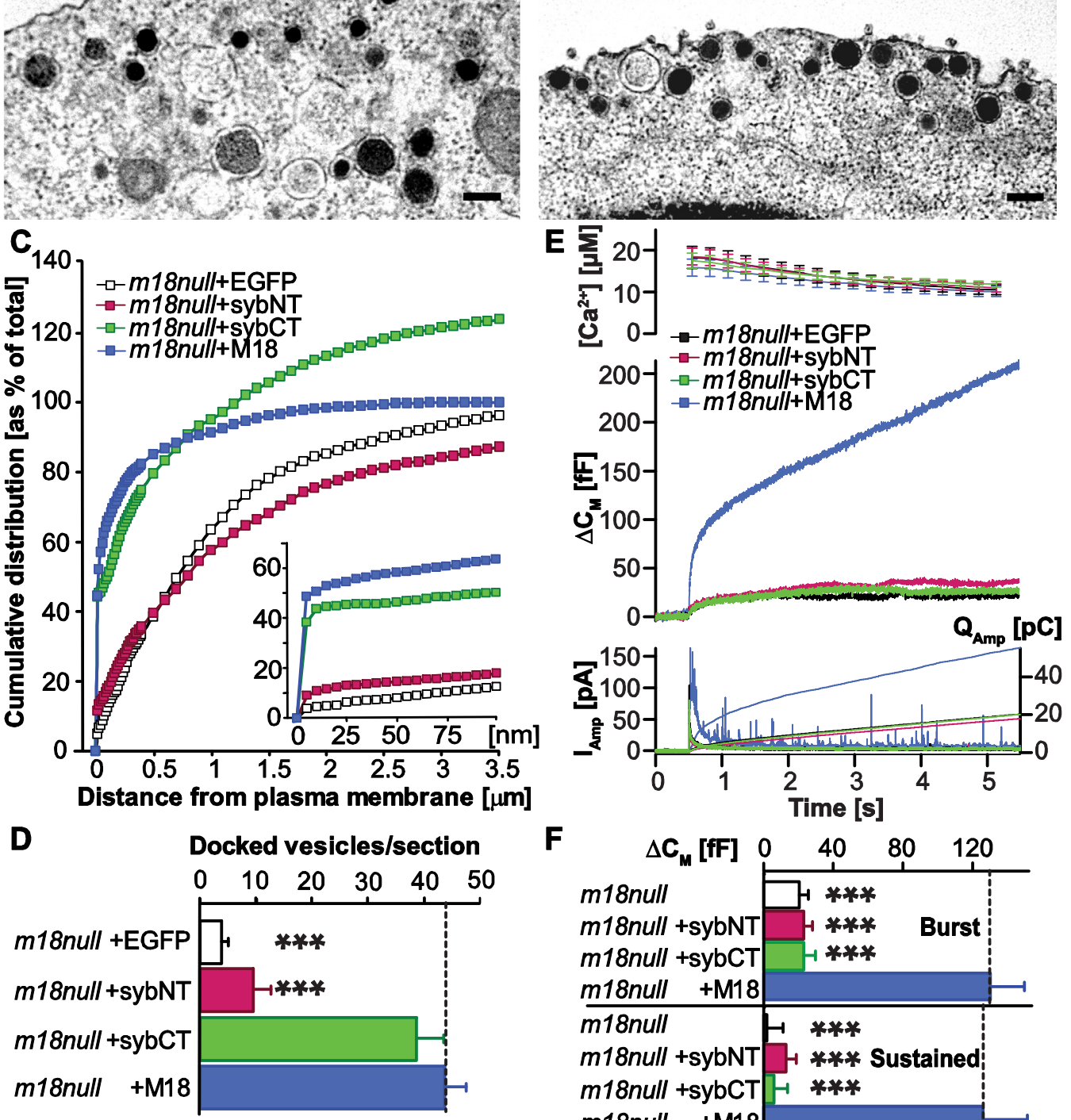

F

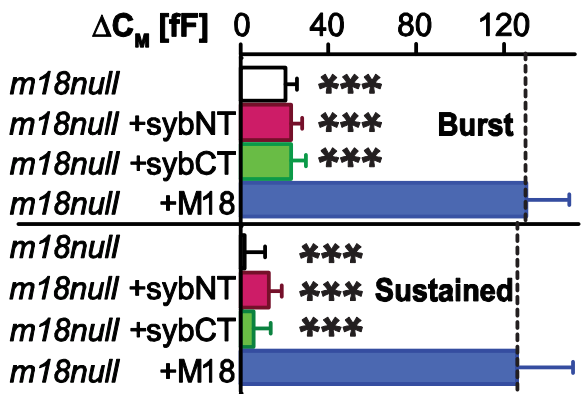

Figure 5. A C-terminal synaptobrevin fragment restores docking but not secretion in absence of Munc18-1. (A and B) Electron micrographs from primary cultured munc18-1 null chromaffin cells 
expressing either a 1-70 N- (sybNT; A) or 49-96 C-terminal (sybCT; B) fragment of synaptobrevin. Bar = $200 \mathrm{~nm}$. (C) Normalized cumulative distribution of vesicles as a function of distance from the plasma membrane. Inset shows cumulative vesicle distribution in the sub-membrane region within 0-100 nm. Data are normalized to control (munc18null+Munc18-1). (D) Number of docked vesicles per section. Abbreviations: sybNT and CT, N-terminal fragment 1-70 and C-terminal fragment 49-96 of synaptobrevin 2. (Data are mean \pm SEM; munc18null+EGFP: $\mathrm{n}=20$; munc18null $+\mathrm{M} 18: \mathrm{n}=19$; munc18null + sybNT $\mathrm{n}=20$; munc18null + sybCT $\mathrm{n}=20$. $* * * \mathrm{p}<0.001$ by Student's $\mathrm{t}$-test compared to control.) Ultrastructural analysis was performed by Heidi de Wit, Center for Neurogenomics and Cognitive Research, Vrije Universiteit Amsterdam, The Netherlands. Modified from de Wit et al. (2009).

$(\mathbf{E}$ and $\mathbf{F})$ Electrophysiological characterization. (E) Average membrane capacitance and amperometric responses. Top panel: mean \pm SEM of intracellular $\mathrm{Ca}^{2+}$-concentration after UV-induced $\mathrm{Ca}^{2+}$-uncaging at time $=0.5 \mathrm{~s}$. Middle panel: mean capacitance increase. Bottom panel: mean amperometric current (thick traces, left ordinate) and amperometric charge (thin traces, right ordinate). (F) Quantification of burst (0-1 s) and sustained (1-5 s) phases of neurotransmitter release in munc18-1 null chromaffin cells and munc18-1null cells expressing Munc-18, sybNT or sybCT. (Data are mean \pm SEM; munc18null: $\mathrm{n}=22$; munc18null $+\mathrm{M} 18: \mathrm{n}=10$; munc18null+sybNT: $\mathrm{n}=25$; munc18null + sybCT: $\mathrm{n}=20$. ${ }^{* * *} \mathrm{p}<0.001$ in Mann-Whitney test compared to controls.)

These observations suggest that a SNAP-25:Syntaxin acceptor complex acts as a docking platform for the association of the secretory vesicle to the plasma membrane.

\subsubsection{Synaptotagmin is a vesicular docking factor}

An important issue in the construction of a model of the molecular processes involved in docking is the identification of the vesicular factor that allows for association of the vesicle to its target membrane. Due to their abundance on the synaptic vesicle and their capability to bind to SNARE partners (Pobbati et al., 2006; Rickman et al., 2006; Takamori et al., 2006) two candidates are especially interesting: the vesicular SNARE protein synaptobrevin 2 and synaptotagmin 1. Although synaptobrevin is essential for the process of transmitter release (Schoch et al., 2001) and had originally been implicated to play a role in docking (Pevsner et al., 1994), docking is normal in chromaffin cells deficient of syb 2 (Gerber et al., 2008) and syb 2/cellubrevin (Borisovska et al., 2005). The study was therefore focused on an analysis of the prime $\mathrm{Ca}^{2+}$ sensor in triggered release, syt 1 (Geppert et al., 1994) and its possible role in vesicle docking. Indeed, cells from knockout mice showed a severe docking defect that could be rescued by viral expression of syt 1 (Figure 6A). This suggests that syt 1 might serve as a vesicular docking protein. 

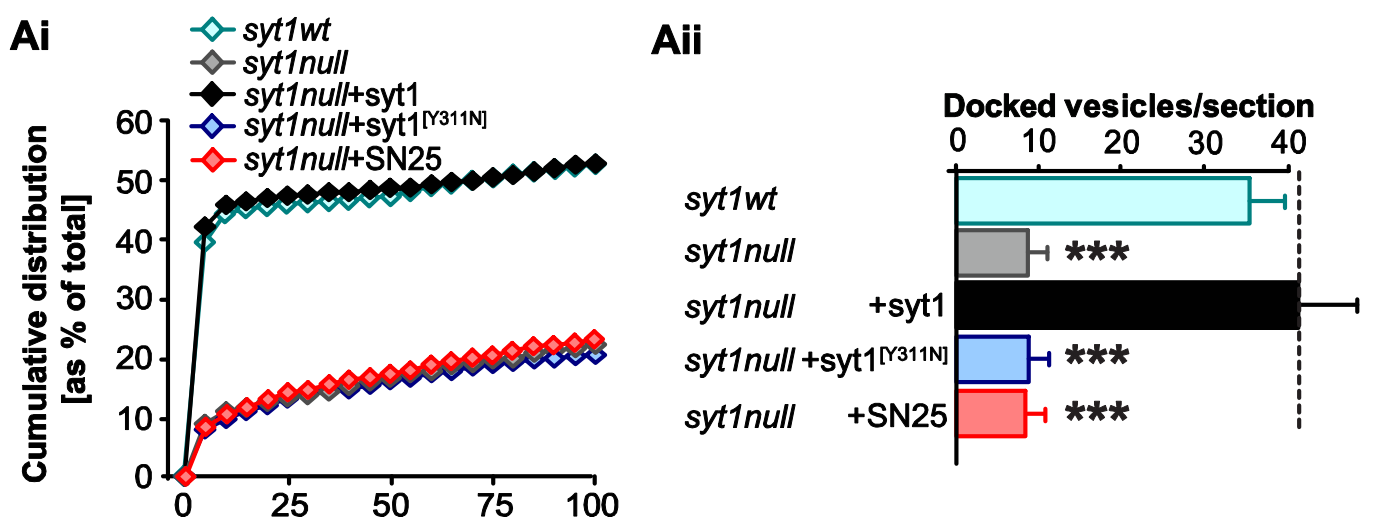

$\mathrm{Bi}$

\section{Distance from plasma membrane [nm]}

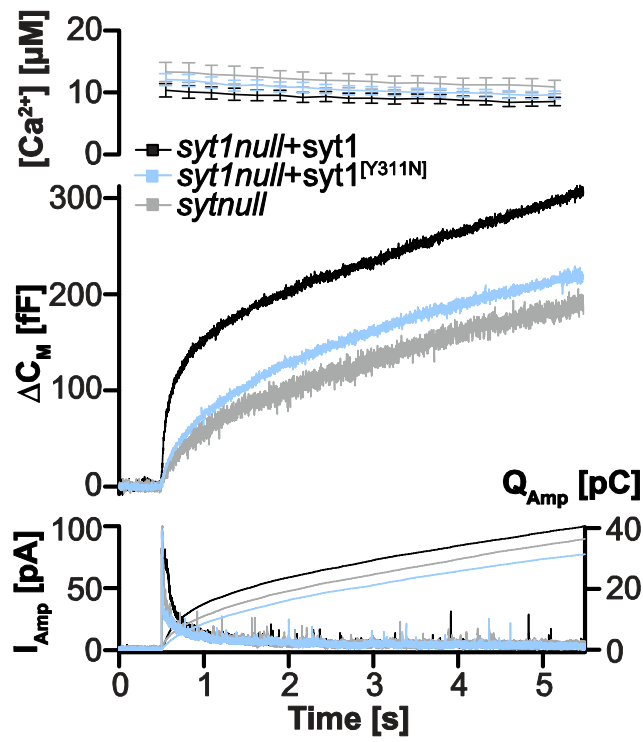

Bii

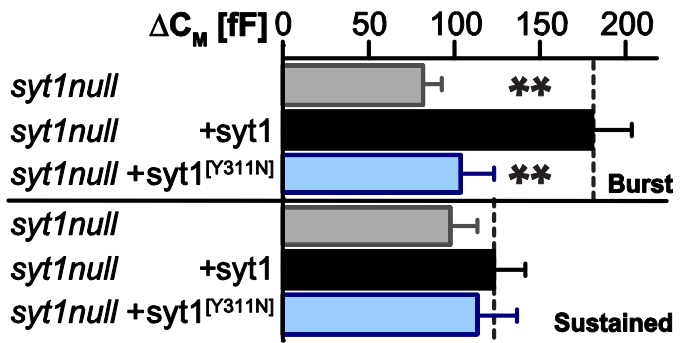

\section{Expression levels}

Fluorescence [A.U.] $\begin{array}{llllll}0 & 5 & 10 & 15 & 20 & 25\end{array}$

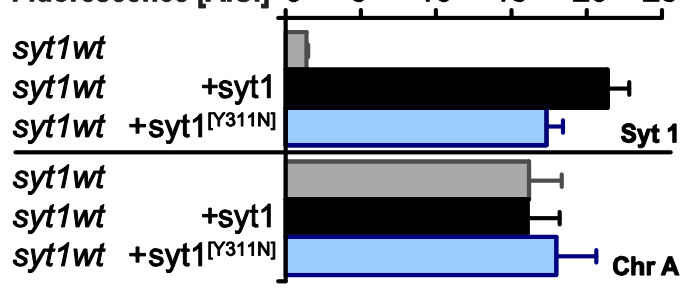

Figure 6. Synaptotagmin-1 binding to SNAP-25 is essential for vesicle docking and fast secretion. (A) Normalized cumulative distribution (Ai) and quantification of docked (Aii) vesicles. (Data are mean \pm SEM; sytnull: $\mathrm{n}=20 ;$ sytnull + syt $1: \mathrm{n}=26 ;$ sytnull + syt $1[\mathrm{Y} 311 \mathrm{~N}]: \mathrm{n}=26 ;$ sytnull $+\mathrm{SNAP}-25: \mathrm{n}=20 .{ }^{* * *} \mathrm{p}<0.001$ by Student's t-test compared to control: syt1null + syt1.) Abbreviations: wt, wild type; syt1, full length synaptotagmin-1; syt1[Y311N], synaptotagmin-1 bearing the Y311N mutation with reduced affinity to SNAP-25 (Rickman et al., 2006); SN25, SNAP-25. Ultrastructural analysis was performed by Heidi de Wit, Center for Neurogenomics and Cognitive Research, Vrije Universiteit Amsterdam, The Netherlands. Modified from de Wit et al. (2009).

(B) Average membrane capacitance and amperometric responses (Bi) and quantification (Bii) of burst- and sustained flash-evoked secretion in syt 1 knockout chromaffin cells. Cells from $\mathrm{KO}$ animals show a reduction in burst size, as do cells from knockout animals expressing syt 1 bearing the [Y311N] mutation. (Data are mean \pm SEM; sytnull: $\mathrm{n}=14$; sytnull + syt $1: \mathrm{n}=13$; sytnull + syt $1[\mathrm{Y} 311 \mathrm{~N}]: \mathrm{n}=24{ }^{* *} \mathrm{p}<0.001$ by Mann-Whitney test compared to control: sytnull + syt 1.)

(C) Expression levels assayed by cumulative fluorescence quantification in wildtype chromaffin (syt $1 w t$ ) and wildtype cells expressing full length syt 1 (syt $1 w t+$ syt1) or the Y311N mutant (syt $1 w t+\operatorname{syt} 1[\mathrm{Y} 311 \mathrm{~N}])$. Virus -driven protein expression of syt1 and syt1[Y311N] are well above wildtype levels. Co-staining with the granular marker Chromogranin A (Chr A) shows no major difference in protein levels. Cells were stained with a primary antibody against syt 1 (Synaptic Systems, Göttingen, Germany) and visualized using a secondary antibody bearing ALEXA546. Co-staining was performed using a Chromogranin A primary antibody (Abcam) and a secondary antibody bearing ALEXA647. For examples obtained by confocal imaging, please refer to Figure 7. 
To test whether syt1 indeed promotes docking by binding to a syntaxin:SNAP-25 acceptor complex, a mutant of syt 1 was used (syt1[Y311N]) that had been reported to have a decreased affinity to SNAP-25 (Rickman et al., 2006). Whereas expression of wildtype syt 1 in knockout chromaffin cells rescued docking, expression of this mutant did not (Figure 6A). Parallel physiological characterization of secretion showed typical responses for knockout $(\mathrm{KO})$ cells (Voets et al., 2001a) where secretion was present, but fast (burst-) secretion was compromised. Expression of syt 1 fully restored the exocytic burst, whereas cells expressing the Y311N mutant essentially phenocopied the KO (Figure 6B). Both constructs (WT rescue and syt 1[Y311]) were highly expressed ( $>10$ fold) and correctly localized (Figure 6C and Figure 7). In the absence of syt 1, overexpression of SNAP-25, unlike the situation in Munc-18 nulls, did not rescue docking, further emphasizing that the defect here is due to the loss of the vesicular docking factor syt 1 (Figure 6A). 

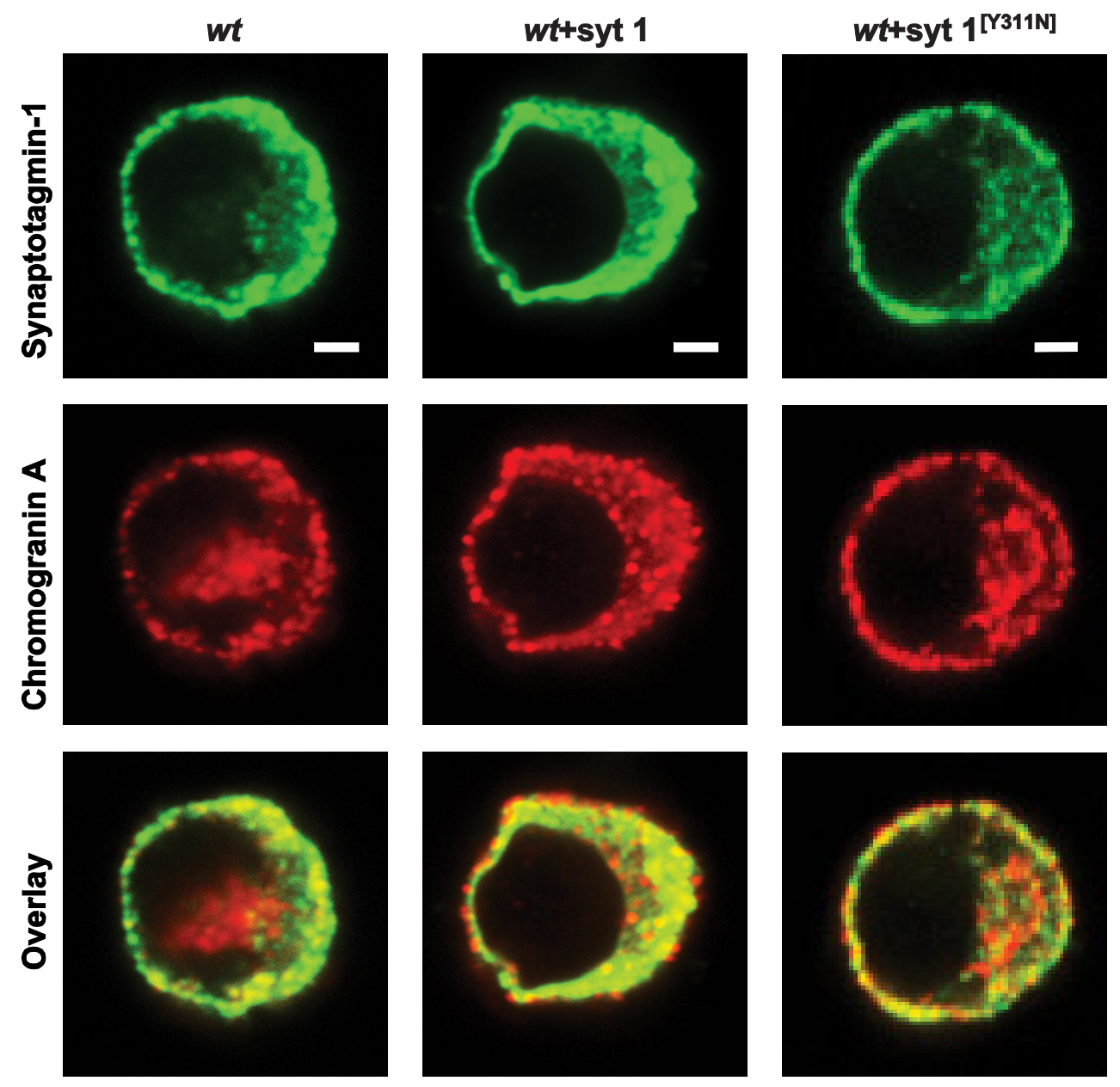

Figure 7. Virally expressed Synaptogamin 1 and syt1[Y311N] colocalize with the granular marker Chromogranin A. Confocal images of wildtype chromaffin cells uninfected $(w t)$ or expressing syt 1 $(w t+\operatorname{syt} 1)$ or $\operatorname{syt} 1[\mathrm{Y} 311 \mathrm{~N}](w t+\mathrm{syt} 1 \mathrm{Y} 311 \mathrm{~N}])$ show colocalization of Synaptotagmin 1 (upper panel) with Chromogranin A (middle panel). Bottom panel: overlay. Scale bar: $2 \mu \mathrm{m}$. Cells were stained with a primary antibody against syt 1 (Synaptic Systems, Göttingen, Germany) and visualized using a secondary antibody bearing ALEXA546. Co-staining was performed using a Chromogranin A primary antibody (Abcam) and a secondary antibody bearing ALEXA647.

An alternative approach was followed by overexpression of the soluble $\mathrm{C} 2 \mathrm{AB}$ domains of syt 1 with the intention of inducing competitive inhibition of endogenous syt 1 . If synaptotagmin indeed were the mediator of docking, attaching the vesicle to the plasma membrane, it would be expected that by blocking its binding site, docking should be reduced. The soluble fragments of synaptotagmin can interfere with the binding site of full-length synaptotagmin, but not promote translocation of the vesicle, simply because they lack the transmembrane domain and therefore are not attached to the vesicle. Viral 
expression of proteins typically induces major overexpression, resulting in protein amounts greatly exceeding the levels of endogenous proteins (Figure 13).
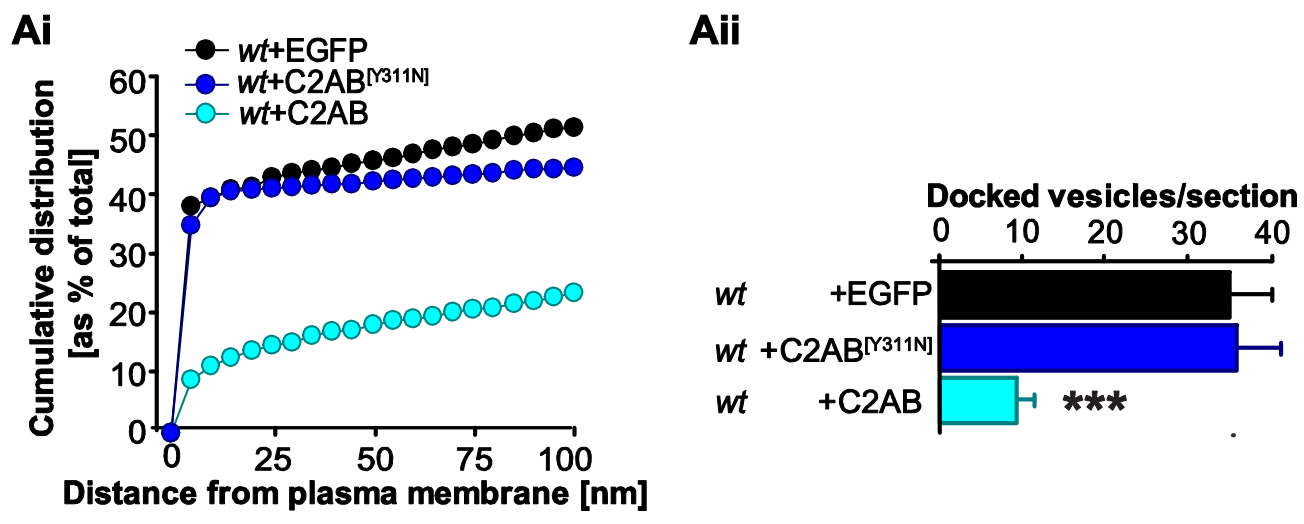

$\mathrm{Bi}$

$\mathrm{Bii}$

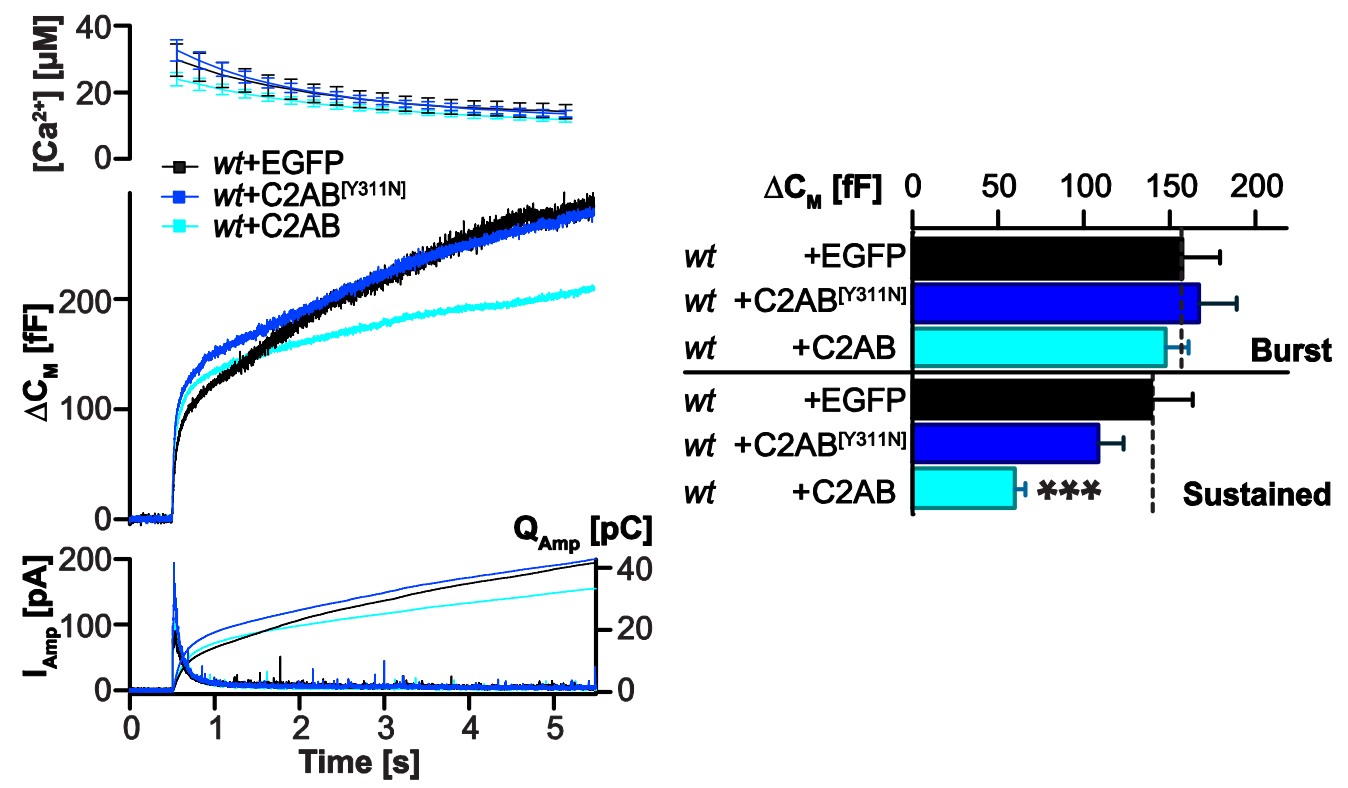

Figure 8. A soluble C2AB domain competing with endogeneous syt 1 affects docking and vesicle recruitment. (A) Normalized cumulative distribution (Ai) and quantification (Aii) of docked vesicles. Abbreviations: wt, wild type; $\mathrm{C} 2 \mathrm{AB}$ and $\mathrm{C} 2 \mathrm{AB}[\mathrm{Y} 311 \mathrm{~N}]$, soluble tandem $\mathrm{C} 2$ domains of synaptotagmin-1 with or without $\mathrm{Y} 311 \mathrm{~N}$ mutation; EGFP, enhanced green fluorescent protein. (Data are mean \pm SEM; $w t+$ EGFP: $\mathrm{n}=21 ; w t+\mathrm{C} 2 \mathrm{AB}: \mathrm{n}=21 ; w t+\mathrm{C} 2 \mathrm{AB}[\mathrm{Y} 311 \mathrm{~N}]: \mathrm{n}=21 . * * * \mathrm{p}<0.001$ by Student's t-test compared to control: $w t+$ EGFP.) Ultrastructural analysis was performed by Heidi de Wit, Center for Neurogenomics and Cognitive Research, Vrije Universiteit Amsterdam, The Netherlands. Modified from de Wit et al. (2009).

(B) Average membrane capacitance and amperometric responses (Bi) and quantification (Bii) of burst- and sustained flash-evoked secretion. Cells expressing a soluble C2AB domain of Synaptotagmin 1 show a reduction of the sustained component. Secretion from cells expressing a soluble $\mathrm{C} 2 \mathrm{AB}$ domain with the $[\mathrm{Y} 311 \mathrm{~N}]$ mutation is not significantly different to secretion from wildtype cells. (Data are mean \pm SEM; $w t+$ EGFP: $\mathrm{n}=20 ; w t+\mathrm{C} 2 \mathrm{AB}: \mathrm{n}=29 ; w t+\mathrm{C} 2 \mathrm{AB}[\mathrm{Y} 311 \mathrm{~N}]: \mathrm{n}=25 . * * * \mathrm{p}<0.001$ by Mann-Whitney test compared to control: $w t+\mathrm{EGFP}$.) 
In fact, expression of wildtype $\mathrm{C} 2 \mathrm{AB}$ domains reduced the number of docked vesicles compared to wildtype cells expressing EGFP (Figure 8A). In contrast, expression of a $\mathrm{C} 2 \mathrm{AB}$ domain bearing the $\mathrm{Y} 311 \mathrm{~N}$ mutation in the $\mathrm{C} 2 \mathrm{~B}$ domain had no effect on the number of docked vesicles suggesting that interaction of the syt 1 C2AB domain with SNAP-25 is essential for vesicle docking. Secretion was affected in cells expressing the soluble wildtype $\mathrm{C} 2 \mathrm{AB}$ domain. Here the sustained component of secretion was significantly reduced (Figure 8B). The sustained phase of exocytosis in flash-evoked release from chromaffin cells corresponds to the release of newly recruited vesicles that immediately undergo fusion when becoming primed due to the sustained high levels of $\mathrm{Ca}^{2+}$. Therefore, a decrease in the sustained component suggests a defect in the steps upstream of release, in agreement with a defect in vesicle recruitment and docking.

Unlike the situation of the synaptotagmin knockout, where a decrease of the exocytic burst was observed, expression of the $\mathrm{C} 2 \mathrm{AB}$ fragment on the wildtype background did not affect the fast phase of secretion. One might expect that the soluble $\mathrm{C} 2 \mathrm{AB}$ fragment should also interfere with the action of synaptotagmin in fast secretion. However, an explanation that may resolve this discrepancy is that burst secretion here may be due to the action of the endogenous syt 1 since these experiments were performed on the wildtype background.

Together, these data allow the building of a minimal working model of the proteins involved and the processes necessary to dock secretory vesicles to the plasma membrane (Figure 9). 


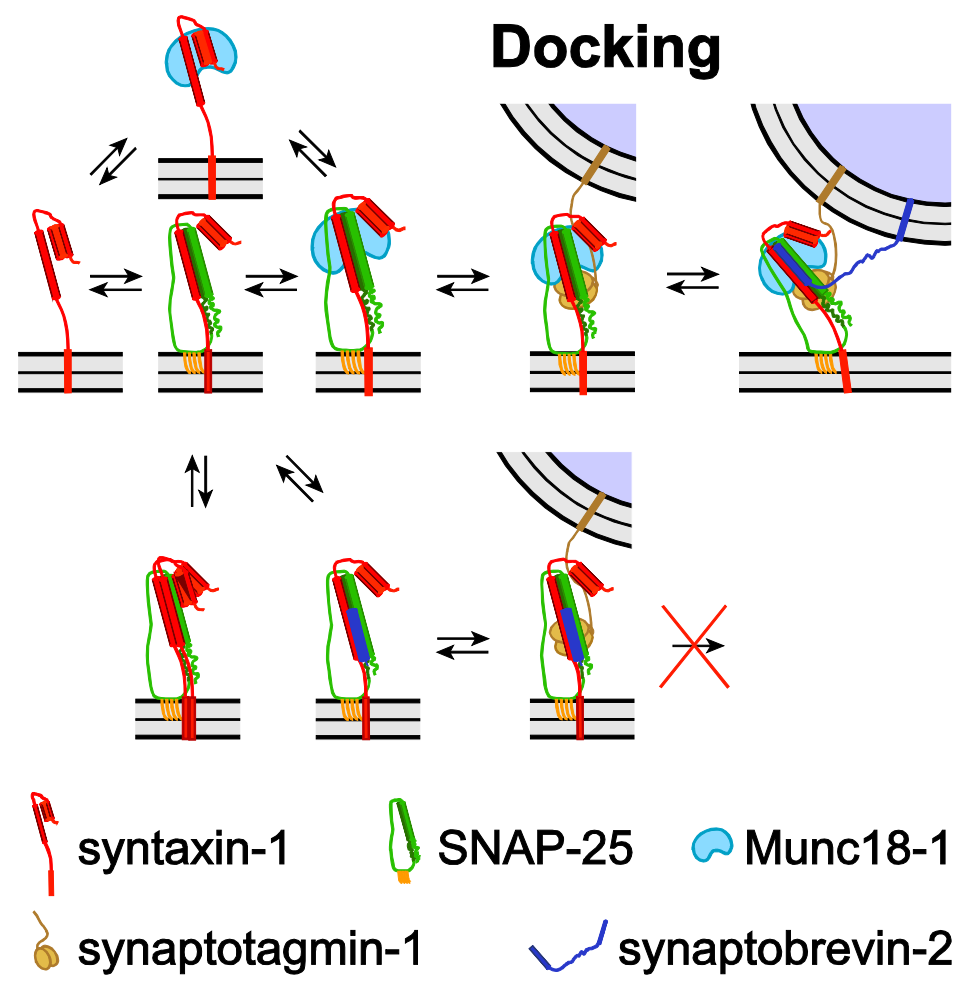

Figure 9. Model of the docking process: syntaxin binds to SNAP-25. This dimer is stabilized by Munc-18 and allows for attachment of the vesicle to the acceptor complex by action of synaptotagmin. In the absence of Munc-18, another syntaxin may bind, thus forming an unproductive 2:1 complex. This can be prevented by excess expression of SNAP-25, or, alternatively by stabilizing the syntaxin:SNAP-25 complex with a Cterminal synaptobrevin fragment. Neither of these alternative pathways, however, renders the vesicle fusion competent. The priming reaction requires Munc-18. (Modified from de Wit et al., 2009) 


\subsection{A partly assembled SNARE complex defines the primed vesicle}

The experiments presented thus far dealt with the investigation of the stable assembly of vesicles at their release site, vesicle docking. These reactions are essentially different from the post-docking functional maturation of vesicles, vesicle priming. This becomes immediately evident when considering above experiments, where manipulations to stabilize the syntaxin:SNAP-25 docking platform in the absence of Munc-18 restored docking but did not suffice to reinstate fusion. This implies that although these reactions are intimately linked (as can be seen by the effect of the $\mathrm{C} 2 \mathrm{AB}$ fragment on the sustained fusion rate in wildtype cells, Figure 8B), docking and priming essentially are distinct reactions. The molecular interactions that link docking to priming and triggering will be addressed next.

The fact that SNAREs are essential and sufficient to drive fusion in-vitro combined with the idea of a reaction clamped in a ready-to-go state in order to match for a rapid release when triggered, lead Sørensen and colleagues to propose the following model (Sorensen et al., 2006): the formation of the SNARE proteins into a complex is responsible for the process of priming and triggering. But complex formation occurs in a sequential manner, where formation of the complex is halted in a meta-stable or cocked state. This would correspond to the process of functional maturation and therefore would be attributed to the priming of vesicles. From here, fusion can be rapid by further, complete assembly of the SNAREs.

Thus, a sequential, two step assembly of the SNARE proteins into a complex could occur as follows: initial N-terminal binding of syb 2 to SNARE partners and partial assembly of the SNARE complex constitutes vesicle priming. Subsequent fusion occurs by final zippering towards the membrane anchors at the C-terminal end, driving fusion in response to triggering (Figure 10). 


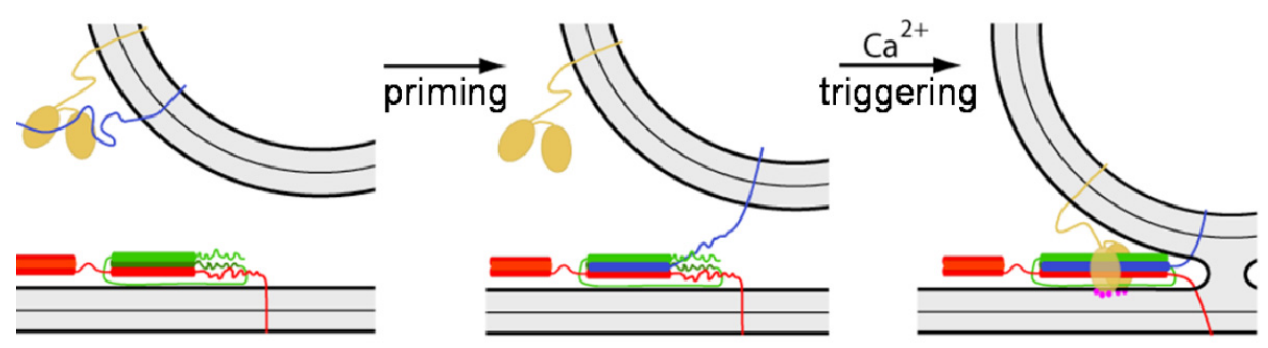

Figure 10. Working hypothesis: sequential zippering of the SNAREs drives priming and fusion. An initial, N-terminal (membrane distal, left) complex assembly of the SNAREs corresponds to vesicle priming. Subsequent $\mathrm{Ca}^{2+}$-dependent $\mathrm{N}$-to $\mathrm{C}$-terminal zippering drives vesicle fusion. Yellow protein: synaptotagmin; blue: synaptobrevin 2; red: syntaxin-1; green: SNAP-25; magenta dots: $\mathrm{Ca}^{2+}$. Modified from Sørensen et al. (2006).

\subsubsection{Rational}

This working hypothesis can be tested by regionally weakening SNARE interaction and characterization of the secretory phenotype. In contrast to models in which SNARE complex assembly occurs in a single step, only under the assumption of sequential binding is it expected that $\mathrm{N}$ - and $\mathrm{C}$-terminal mutants yield different phenotypes.

\subsubsection{Mutants in synaptobrevin with regional destabilization}

In order to test the hypothesis of sequential SNARE complex assembly, a mutagenesis approach was employed. SNARE interaction was weakened by introduction of destabilizing point mutations in synaptobrevin, specifically targeting amino acids contributing to the hydrophobic layers (Figure 11). In most cases alanine substitutions were used. Considering the known structure of the SNARE-complex, alanines are not expected to prevent the binding of synaptobrevin to SNARE partners, or cause changes in the structure, but most likely will mildly destabilize the complex. This assumption was later confirmed by biochemical measurements of complex stability and heat of formation (see section below [5.2.5]). The effect of these mutations on the rate and affinity of complex assembly was tested in-vitro and the effect on neurotransmitter release was tested in vivo. 


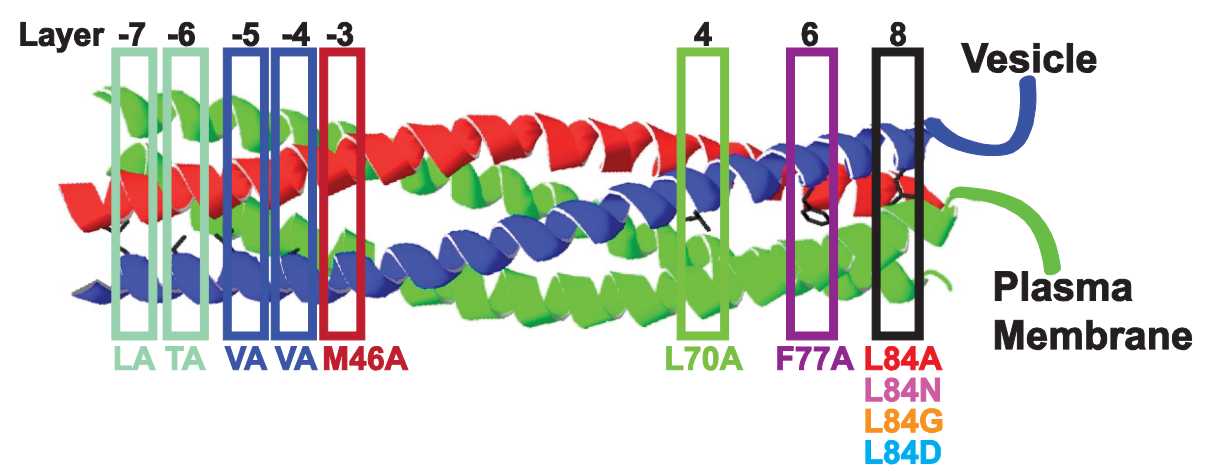

Figure 11. Overview of mutations introduced into synaptobrevin 2 in the present investigation. All targeted sites were 'layer' residues facing the inside of the complex (Fasshauer et al., 1998) either in the Nterminal end (left), or in the C-terminal end (right) of the complex. In the "LATA"-mutant amino acids 32 (leucine) and 35 (threonine) corresponding to layers -7 and -6 were mutated to alanine. The "VAVA" mutant bears mutations in layers -5 and -4 where valines (residues 39 and 42 ) were mutated to alanines. In layer -3 methionine 46 was replaced by alanine (M46A). In the C-terminal half of the complex, leucine 70 was mutated to alanine (L70A) and phenylalanine to alanine (F77A) in layers +4 and +6 , respectively. In layer +8 leucine was substituted by alanine (L84A), arginine (L84N), glycine (L84G), or aspartate (L84D).

To examine possible regional sensitivity to destabilization of the SNARE complex, two main sets of mutations were investigated at the $\mathrm{N}$ - and $\mathrm{C}$-terminal end of the SNARE motif. Two mutants bearing double point mutations at the Nterminal were generated. Mutating residues leucine-32 (L) and threonin -35 (T) of layers -7 and -6 of syb 2 yielded the "LATA" mutant, whereas mutation of valines 39 and 42 in layers -5 and -4 created the "VAVA" mutant; a single point mutant was created in the adjacent layer -3 , where methionine 46 was replaced by alanine, thus creating the M46A mutant (Figure 11).

Contribution of the C-terminal end of the complex was assessed by mutations in layers $+4,+6$ and +8 , where alanines were used to replace a leucine (L70A), a phenylalanine (F77A) and a leucine (L84A), respectively.

In order to test for a regio-sensitivity of SNARE destabilization, mutants were characterized electrophysiologically. Flash-evoked secretion was monitored by whole cell capacitance measurements (Lindau and Neher, 1988) combined with amperometry (Wightman et al., 1991). Following stimulation, vesicles fuse and the observed capacitance increase shows multi-exponential behavior. This is thought to be due to fusion of separate kinetic pools of vesicles (i.e. the readily releasable pool (RRP) and the slowly releasable pool (SRP)) (Voets, 2000). 
Individual capacitance traces can be kinetically analyzed by the fitting of multiple (double- or triple-) exponential functions, allowing extraction of parameters describing sizes (poolsizes) and kinetics (time constants) of the RRP and SRP (Sorensen et al., 2003b). Subsequent to the depletion of RRP and SRP, exocytosis persists at a sustained rate, due to fusion of newly primed vesicles as long as the $\mathrm{Ca}^{2+}$ concentration remains high.

In order to provide a clean genetic background for our in vivo studies viral protein overexpression was performed in cells deficient of synaptobrevin 2 (syb 2) and cellubrevin, since in chromaffin cells cellubrevin partly compensates for synaptobrevin in its absence (Borisovska et al., 2005). Secretion in these cells was greatly impaired but could be restored upon viral overexpression of the syb 2 protein. In fact, expression of syb 2 in double knockout (DKO) cells completely rescued secretion to wildtype levels (Figure 12A).

A

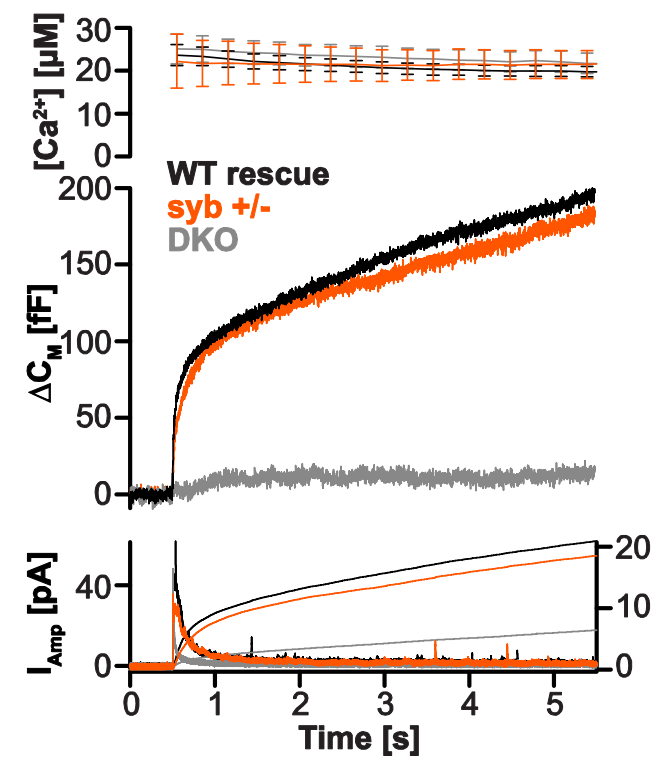

B

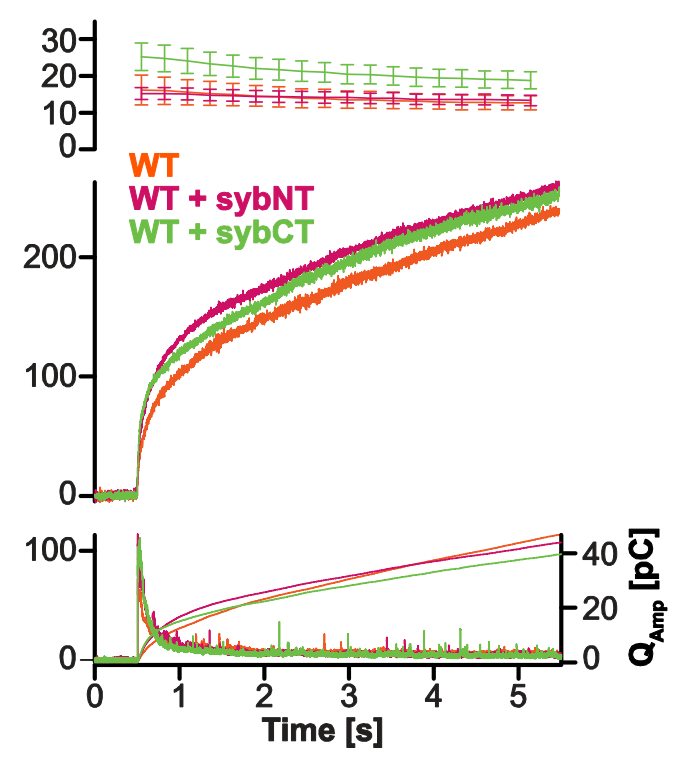

Figure 12. Viral expression of synaptobrevin 2 in syb/ceb double knockout cells can fully rescue secretion. Release is unaffected by expression of syb 2 fragments in wildtype cells. (A and B). Average capacitance and amperometric responses in flash- evoked transmitter release. Top panel: mean \pm SEM of intracellular $\mathrm{Ca}^{2+}$-concentration after $\mathrm{UV}$-induced $\mathrm{Ca}^{2+}$-uncaging at time $=0.5 \mathrm{~s}$. Middle panel: mean capacitance increase. Bottom panel: mean amperometric current (thick traces, left ordinate) and amperometric charge (thin traces, right ordinate). (A) Chromaffin cells deficient of syb 2 and cellubrevin (DKO) show a severe defect in secretion which can be rescued by viral expression of syb 2 (WT rescue). WT rescue in knockout cells restores secretion to levels of heterozygous mice $(\mathrm{syb}+/-)$ which are indistinguishable from wildtype (Borisovska et al., 2005). (B) Expression of an N-terminal (1-70, sybtNT)- or C-terminal (49-96, sybCT) fragment of synaptobrevin 2 on a wildtype background does not affect secretion. (A, WT rescue: $\mathrm{n}=30$; syb +/-: n=13, DKO: n=9. B, WT: $\mathrm{n}=19$; WT+SybNT: $n=25 ;$ WT+SybCT: $\mathrm{n}=20$.) 
Expression of $\mathrm{N}$ - and $\mathrm{C}$-terminal fragments of synaptobrevin that were shown to affect docking in the absence of Munc-18, but unable to restore secretion under these conditions (Figure 5), did not affect secretion when expressed in wildtype chromaffin cells (Figure 12B). This suggests that unlike the situation in-vitro, where the speed of syb 2 binding to a syntaxin:SNAP-25 acceptor complex could be greatly increased, the reaction in vivo is already optimized for speed by the catalytic action of additional factors, possibly Munc- 18 .

Experiments testing the effect of specific mutations on the process of transmitter release were performed in a strictly parallel fashion: DKO cells expressing the mutant construct were compared to DKO cells from the same preparation expressing syb 2 (referred to as WT rescue in the following). All constructs led to more than tenfold expression over wildtype levels and were correctly localized, as shown by immunocytochemistry, quantitative fluorescence measurements and confocal imaging (Figure 13, for the M46A mutant also see Figure 15B). 

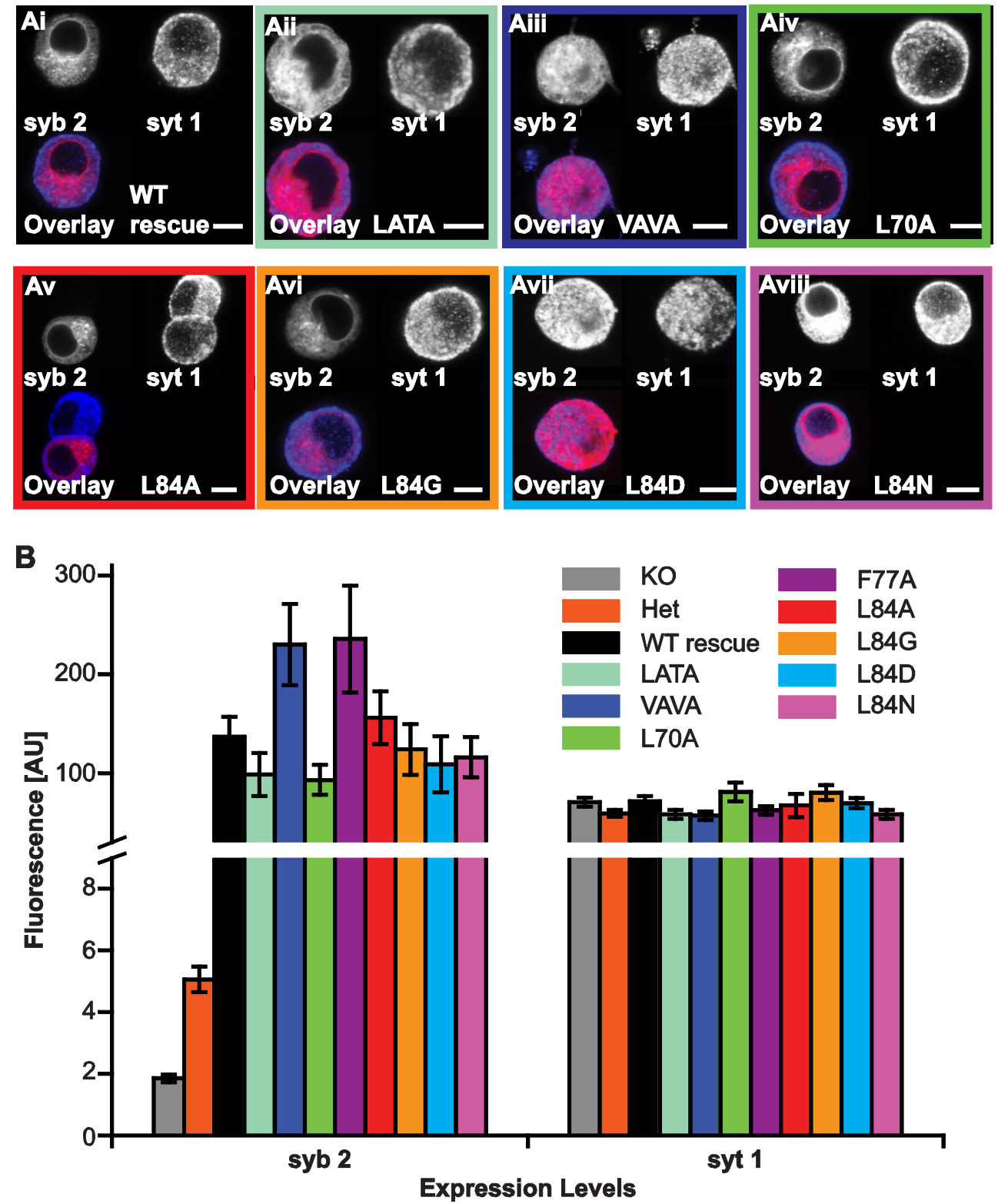

Figure 13. Mutants were correctly localized and expressed in sufficient amounts. (A) Confocal images of fixed chromaffin cells stained for synaptobrevin 2 (syb 2, visualized by secondary antibody bearing Alexa546) and synaptotagmin 1 (syt 1) (visualized by Alexa647). (Ai-Aviii) Images of knockout cells expressing syb 2 wildtype protein (WT rescue (Ai)) or LATA (Aii), VAVA (Aiii), L70A (Aiv), L84A (Av, note that this image contains two attached cells of which one expresses the virus and is positive for syb. The uninfected cell is negative for syb, but positive for syt), L84G (Avi), L84D (Avii) and L84N (Aviii) mutations. First channel syb 2, second channel syt 1, third channel overlay. Scale bar $5 \mu \mathrm{m}$. (B) Quantification of expression levels by fluorescence intensity (widefield fluorescence, integrated fluorescence over the whole cell). (For analysis of the M46A mutant, please refer to Figure 15.) 


\subsubsection{N-terminal destabilization affects vesicle priming}

Mutants of syb 2 challenging N-terminal SNARE complex stability showed partial rescue of secretion, but the overall amplitude of the response was reduced in the "LATA" and "VAVA" mutants (Figure 14Ai). A more detailed view of the kinetics of release was obtained by normalization of the three traces (wildtype, "LATA" and "VAVA") to the same amplitude at $0.5 \mathrm{~s}$ after calcium photorelease. The fact that all three traces overlap after normalization demonstrates that the phenotype observed here is selective for the magnitude, but without effect on the kinetics of release itself (Figure 14Aii). This was also shown by a kinetic analysis of capacitance traces, providing parameters describing the distinct pools (Figure 14Bi, 14Bii). Here, the number of vesicles residing in the RRP and the SRP as well as the rate of the sustained component, which reflects the speed at which vesicles undergo functional maturation, was significantly reduced. However, the kinetics (time constants) of the RRP and SRP were not affected by $\mathrm{N}$-terminal destabilization, confirming that the N-terminal part of the SNARE motif is exclusively responsible for vesicle priming but insignificant for the actual process of triggering release.

Ai

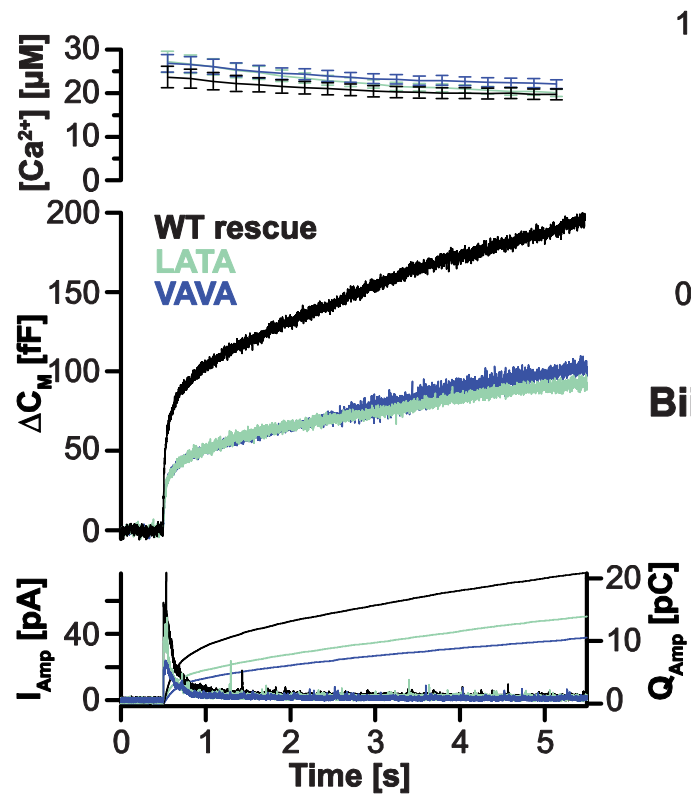

Aii

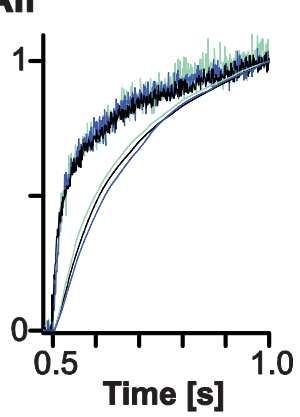

$\mathrm{Bi}$

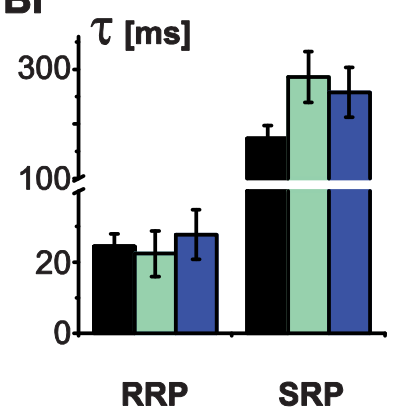

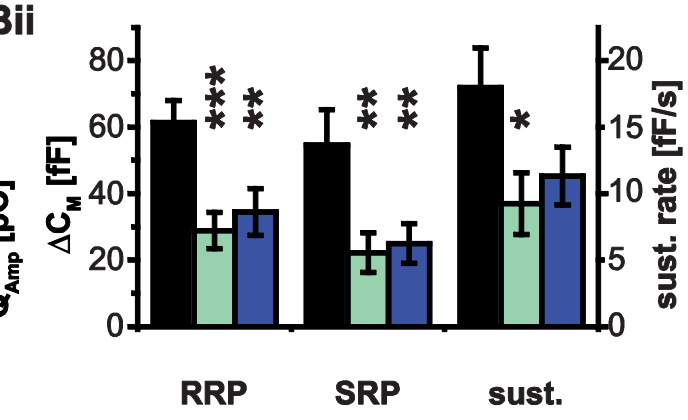

Figure 14. N-terminal mutants of synaptobrevin 2 (see Fig. 11) reduce pool size and sustained component without effect on burst kinetics. (Ai) N-terminal SNARE destabilization shows a reduced secretory response in exocytosis evoked by calcium uncaging. All cells were from double 
synaptobrevin 2/cellubrevin knockout mice; control cells were infected with the wildtype protein (black, WT rescue). (Aii) A detailed view of normalized traces in (Ai) shows that kinetics of release is unaffected (normalized capacitance: thick traces, normalized amperometric charge: thin traces). (Bi and Bii) Kinetic analysis reveals significant reduction of pool sizes in LATA and VAVA mutants but no effect on speed of release. Fitting of exponentials to capacitance responses was used to quantify parameters reflecting time constants (Bi) and sizes (Bii) of the readily releasable pool (RRP) and slowly releasable pool (SRP), as well as the sustained component (sustained). (Data are mean \pm SEM; WT rescue: $n=21$; LATA: $n=20$; VAVA: $\mathrm{n}=29 . *: \mathrm{p}<0.05 ; * *: \mathrm{p}<0.01 ; * * * \mathrm{p}<0.001$ in $\mathrm{t}$-test.)

The M46A mutant in layer -3 also showed a reduced response in triggered exocytosis compared to wildtype rescue (Figure 15Ai). However, secretion was partly rescued compared to double knockout (DKO) cells that were recorded in parallel. Again, kinetics of release was unaffected, demonstrated by the overlapping normalized traces of M46A mutant and control (Figure 15Aii). Similar to the effect observed in the "LATA" and "VAVA" mutant, this mutation suggests a selective effect of N-terminal destabilization on poolsize, but not on the speed of release. Due to the very small amplitude of the secretory response in this particular mutant and the problems that arise from this with respect to a kinetic analysis (see discussion), these cells were not analyzed by fitting exponentials. Instead, the quantification was restricted to the membrane capacitance of the cell prior to stimulation (a measure of the cell size) and the size of the burst (first second of release, in wildtype cells roughly corresponding to the sum of the RRP and SRP) as well as the sustained component (Figure 15C). This examination revealed that cells expressing the M46A mutant show a highly significant reduction in parameters reflecting the burst and sustained phase of transmitter release when compared to wildtype overexpression. Nevertheless, there was significant rescue of the DKO phenotype and protein levels of the mutant were similar to levels of the wildtype rescue (Figure 15B). In these experiments it was additionally seen that DKO cell size prior to stimulation of exocytosis was reduced. This is consistent with the idea that SNARE dependent membrane fusion had occurred prior to flash photolysis. 


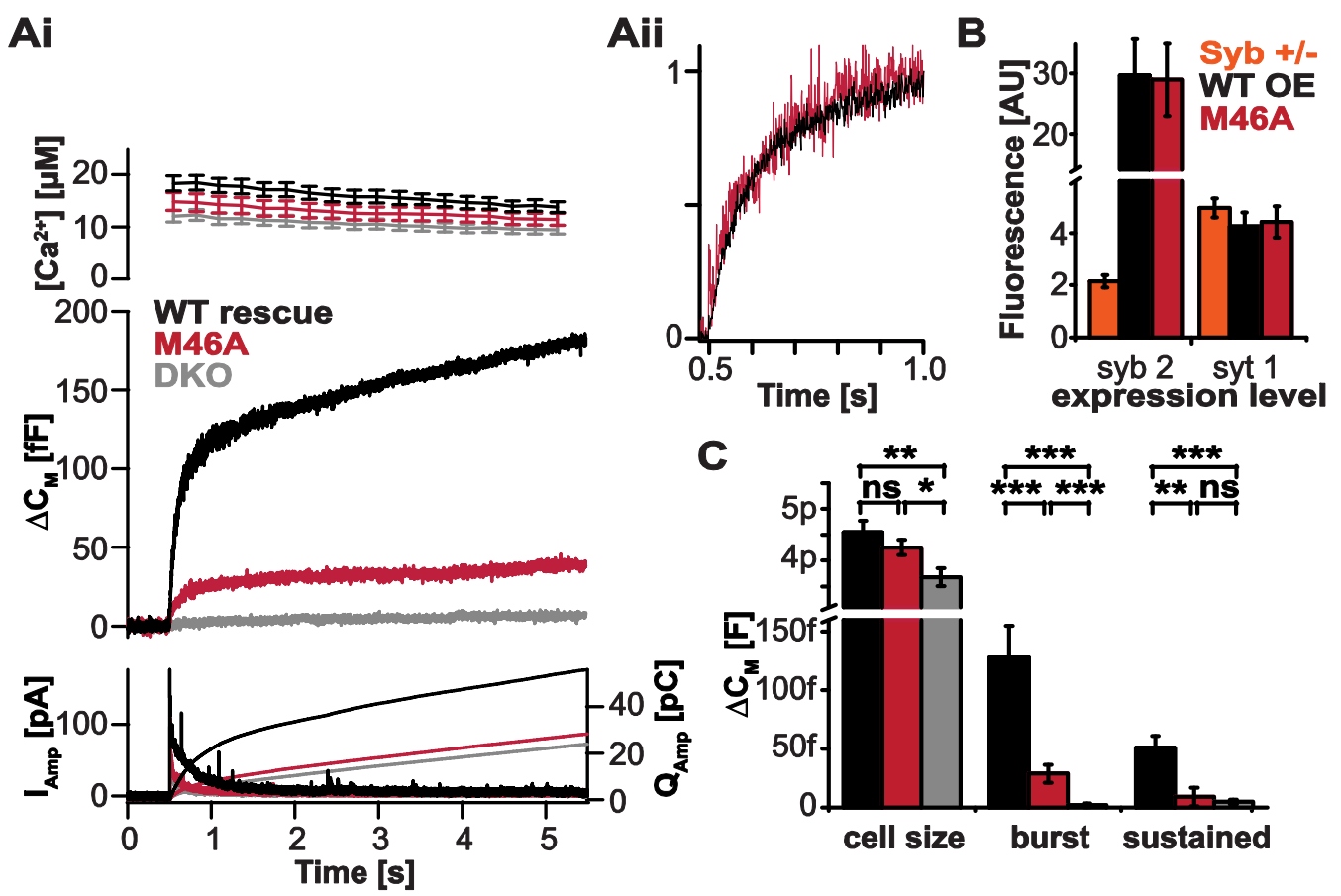

Figure 15. Destabilization of layer $\mathbf{- 3}$ (see Fig. 11) severely affects secretion by decreasing poolsize without apparent effect on release kinetics. (Ai) The M46A mutant shows a reduced secretory response in exocytosis evoked by calcium uncaging. (Aii) A detailed view of normalized capacitance traces in (Ai) shows that kinetics of release is unaffected. (B) Expression levels assayed by quantitative immunocytochemistry show similar protein levels in cells expressing the M46A mutant as in WT rescue. Chromaffin cells were fixed and stained for synaptobrevin 2 (visualized by secondary antibody bearing Alexa546) and Synaptotagmin 1 (Syt 1) (visualized by Alexa647). (C) Quantification of cellular capacitance prior to stimulation (cell size) as well as burst and sustained secretion show that all release phases are affected by the M46A mutation. (Data are mean \pm SEM; WT rescue: $\mathrm{n}=11$; M46A: $\mathrm{n}=17$; DKO: $\mathrm{n}=15$. $^{*}: \mathrm{p}<0.05$; **: $\mathrm{p}<0.01$; $* * *: \mathrm{p}<0.001$ in Mann-Whitney test.)

\subsection{4 $\mathrm{C}$-terminal assembly occurs after triggering}

In order to test how assembly of the C-terminal half of the SNARE complex is involved in vesicular release, a number of mutants were tested. Introducing point mutations layer by layer, I progressively moved from layer +4 towards the $\mathrm{C}$ terminal end of the SNARE motif, again seeking to destabilize SNARE interaction by replacing endogenous residues with alanines. Single point mutations in layers +4 and +6 , where alanines were used to replace a leucine (L70A) and a phenylalanine (F77A) had dramatic effects on exocytosis (Figure 16): both mutants led to only marginal rescue of secretion in double knockout cells, in spite of being strongly overexpressed (Figure 13). 
Ai

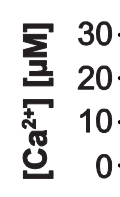
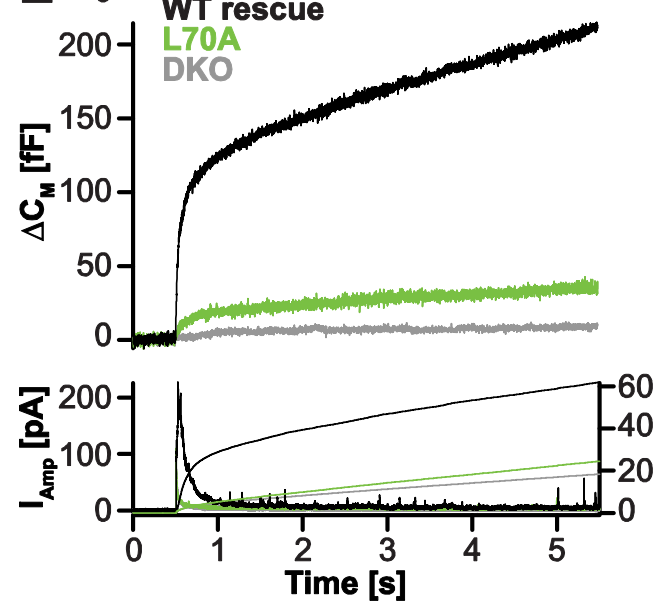

Aii

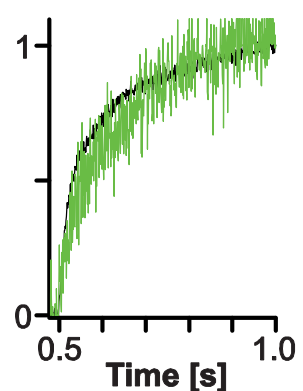

Bi
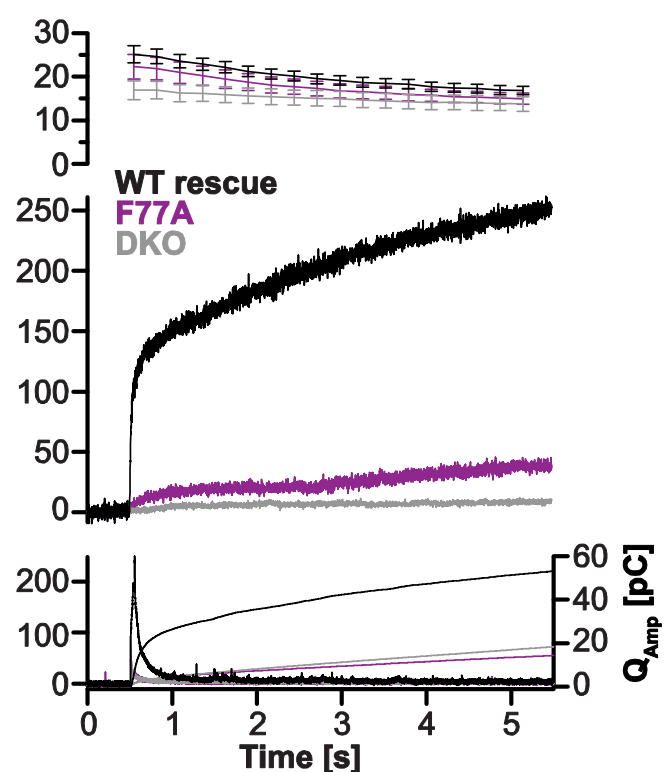

Bii

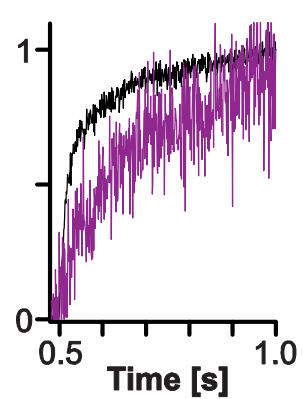

Figure 16. Mutations in layers +4 and +6 (see Fig. 11) severely affect transmitter release. (Ai and $\mathrm{Bi}$ ) Average secretory responses in $\mathrm{Ca}^{2+}$ triggered release are dramatically reduced in the L70A (Ai) and the F77A (Bi) mutant. For comparison, traces from double knockout cells (DKO) are included. DKO traces are pooled from an independent dataset and were not recorded in parallel to L70A and F77A measurements. (Aii and Bii) Detailed view of normalized capacitance traces in (Ai and Bi). (A: WT rescue $n=23$; L70A: $n=15$; DKO $n=21$. B: WT rescue $n=8$; F77A n=13; DKO: $n=21$.)

Strikingly, overexpression of the L70A construct in wildtype chromaffin cells was without effect on secretion (Figure 17), suggesting that mutation rendered the mutant proteins incapable of competing with endogenous syb 2 . 


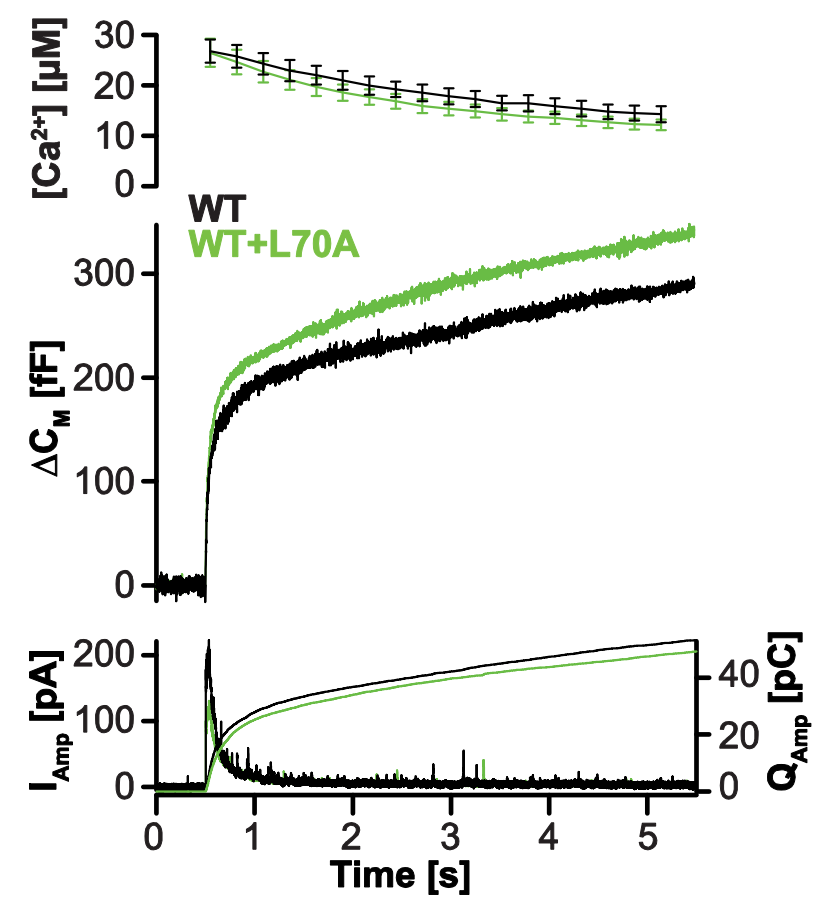

Figure 17. The layer +4 mutant $($ L70A) does not act as a dominant negative on a wildtype background. Average secretory response in $\mathrm{Ca}^{2+}$ triggered release is unaffected by expression of the layer +4 mutant in wildtype cells. (WT: wildtype cells $n=6$ cells; $W T+L 70 A n=13$ cells.)

In contrast, mutating the very C-terminal layer +8 (leucine-84) to alanine (Figure 11) resulted in a phenotype with robust rescue of secretion in response to $\mathrm{Ca}^{2+}$-uncaging (Figure 18Ai). However, detailed analysis of capacitance increase and cumulative amperometric current revealed a slowdown of the fast phase of neurotransmitter release (Figure 18Aii, Aiii). The specific nature of the residue used to replace the endogenous leucine was of minor importance, since a number of different amino acids were studied, all of which showed a similar effect. Even though mutants bearing an aspartate or a glycine in layer +8 also had an effect on the amplitude of the secretory response (Figure 18Bi), it is striking that their effect on release kinetics was very similar and, when looking at normalized traces, even indistinguishable (Figure 18Bii, Biii). Hence, destabilizing SNARE-interaction in layer +8 predominantly reduced the speed at which vesicles undergo fusion suggesting that the extreme C-terminal interaction layer of the SNARE proteins is of particular importance for the very final steps of triggered exocytosis. 
Ai

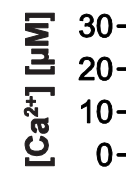
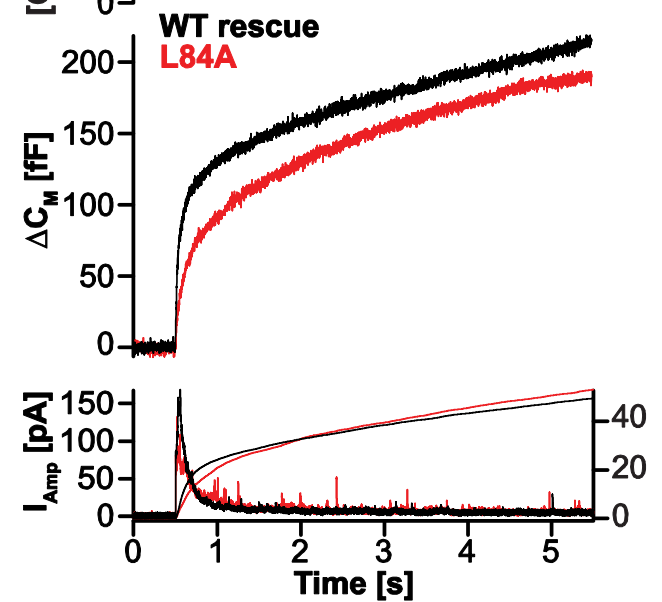

Aii

Aiii

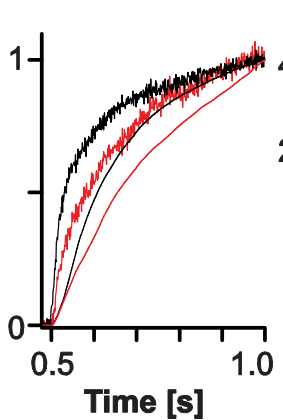

Time [s]

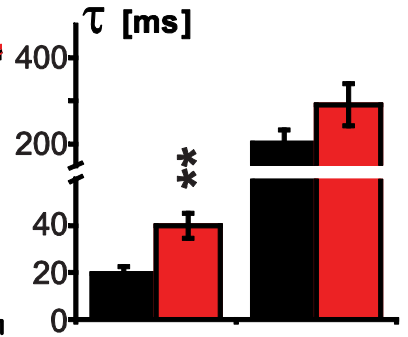

RRP

SRP
Bi

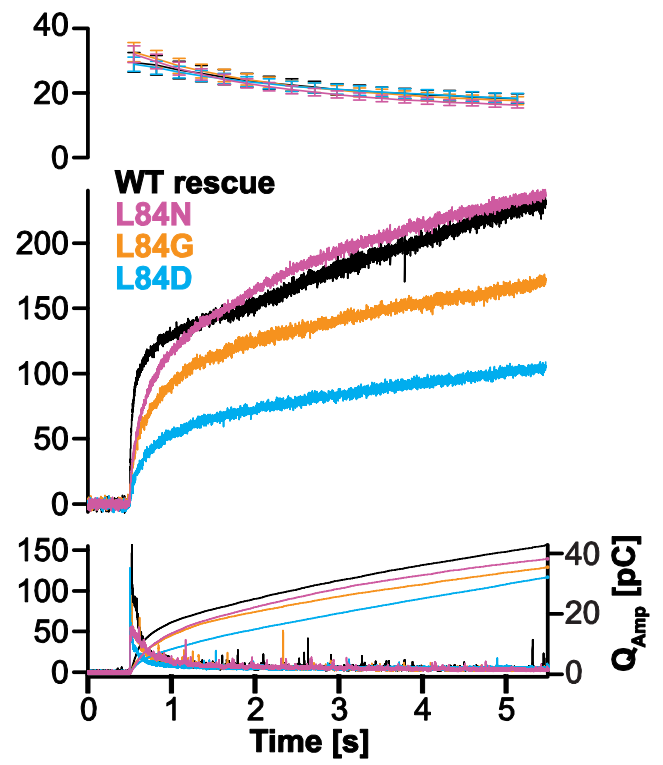

Bii

Biii

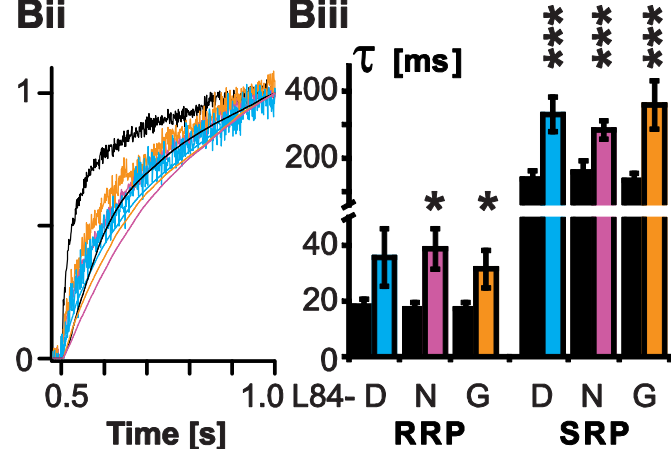

Figure 18. C-terminal mutants of synaptobrevin 2 (see Fig. 11) cause a slowdown of transmitter release. (Ai and Bi) Average secretion in flash-evoked transmitter release is slowed down. (Aii and Bii) Detailed views of normalized traces in (Ai and Bi) show that kinetics of release is slowed down in all cases. (Aiii and Biii) Time constants of the RRP and SRP obtained by fitting of exponentials show slowdown of release in the L84A mutant (Aiii, WT rescue: $\mathrm{n}=19$; $\mathrm{L} 84 \mathrm{~A}: \mathrm{n}=25$ ) and $\mathrm{L} 84 \mathrm{~N}$, L84G and L84D mutations (Biii, WT rescue: $\mathrm{n}>15$; L84D: $\mathrm{n}=9 / 14$; L84G $\mathrm{n}=13 / 17$; L84N n=15/19, for RRP/SRP time constants. *: $\mathrm{p}<0.05$; **: $\mathrm{p}<0.01$; $* * *: \mathrm{p}<0.001$ in t-test ).

\subsubsection{Biophysical characterization}

In order to gain mechanistic insights into synaptobrevin action it is not only important to study its role in secretion following destabilization but also to study the direct effect of the alterations on the binding to SNARE partners. These experiments can be best performed on the isolated proteins in-vitro. It is of particular interest to characterize how mutations affect the kinetics and the thermodynamics of the reaction. In addition it is necessary to prove that the 
mutants studied here all form stable complexes, as this is a requirement for all subsequent interpretations.

Assembly of the trimeric SNARE complex must involve at least two binding steps. The initial step is the assembly of SNAP-25 and syntaxin to a 1:1 acceptor complex, followed by assembly of syb 2 from the vesicle (Fasshauer, 2003). Many features of the SNARE complex, as well as the interaction of the proteins involved, have been thoroughly studied in-vitro. However, relevance to the situation in vivo has been difficult to estabish since two major parameters did not match: the kinetics and the $\mathrm{Ca}^{2+}$ sensitivity. It has been found that one of the major complications of in-vitro studies is the formation of an unproductive 2:1 syntaxin:SNAP-25 complex (off pathway) (Fasshauer and Margittai, 2004). Two strategies were employed and shown to greatly accelerate SNARE complex assembly and liposome fusion in-vitro: shifting the equilibrium concentration of the syntaxin:SNAP-25 complex towards 1:1 in excess of SNAP-25 by law of mass action, or, stabilizing the 1:1 conformation by addition of a C-terminal fragment of synaptobrevin that stabilized the dimer (generating the so-called $\Delta \mathrm{N}$-comlex) (Pobbati et al., 2006). This suggests that SNARE complex formation in-vitro is initiated by syb 2 binding via its $\mathrm{N}$-terminus and subsequent $\mathrm{N}$ - to $\mathrm{C}$-terminal zippering. Especially the greater compliance of the reaction rate with the situation in vivo renders this experimental paradigm the most powerful in-vitro assay to study SNARE complex assembly.

Following this notion anisotropy measurements were performed and the speed of synaptobrevin binding to a $\Delta \mathrm{N}$ acceptor complex was analyzed. Destabilization of the two most N-terminal layers ("LATA"-mutant) of the SNARE-complex reduced the speed of synaptobrevin binding to the acceptor complex, whereas no effect could be seen by mutations at the C-terminal end (L84A) (Figure 19A). Titrating with increasing amounts of $\Delta \mathrm{N}$-complex (Figure 19C), the association rate constant was estimated to be $500000 \mathrm{M}^{-1} \mathrm{~s}^{-1}$ for wildtype synaptobrevin, in agreement with recent findings (Pobbati et al., 2006). This rate was decreased $>10$-fold by the "LATA"-mutant (44000 $\left.\mathrm{M}^{-1} \mathrm{~s}^{-1}\right)$, and slightly reduced for the "VAVA"-mutant (330 $000 \mathrm{M}^{-1} \mathrm{~s}^{-1}$ ). Surprisingly, the effect of the "VAVA"- 
mutant in this particular experimental paradigm was subtle and deviated from the harsh effect of the "LATA" mutant. In vivo, however, these phenotypes were indistinguishable (Figure 14). Possibly, the actual rate-limiting step in vivo was inaccessible by the in-vitro approach. I suspected that the binding of the first two layers (-7 and -6) in-vitro might have been sufficient for the initiation of complex assembly under these conditions thereby masking the effect of the "VAVA" mutant. In order to test this hypothesis and since I continued to suppose that residues 39 and 42 play an important role in vesicle priming, the binding of the "VAVA" mutant in absence of the first two layers was characterized. Deletion of the first two layers indeed greatly slowed down the binding of the Synaptobrevin protein $(\Delta 32-35 \mathrm{Syb}$, Figure 19A, B). Moreover, the combination of deletion and mutations in layers -5 and $-4(\Delta 32-35 \mathrm{VAVA})$ reduced the speed of complex assembly even further (Figure 19B), once again demonstrating the crucial role of the N-terminal layers for the initial binding of SNARE partners in-vitro and in vivo.
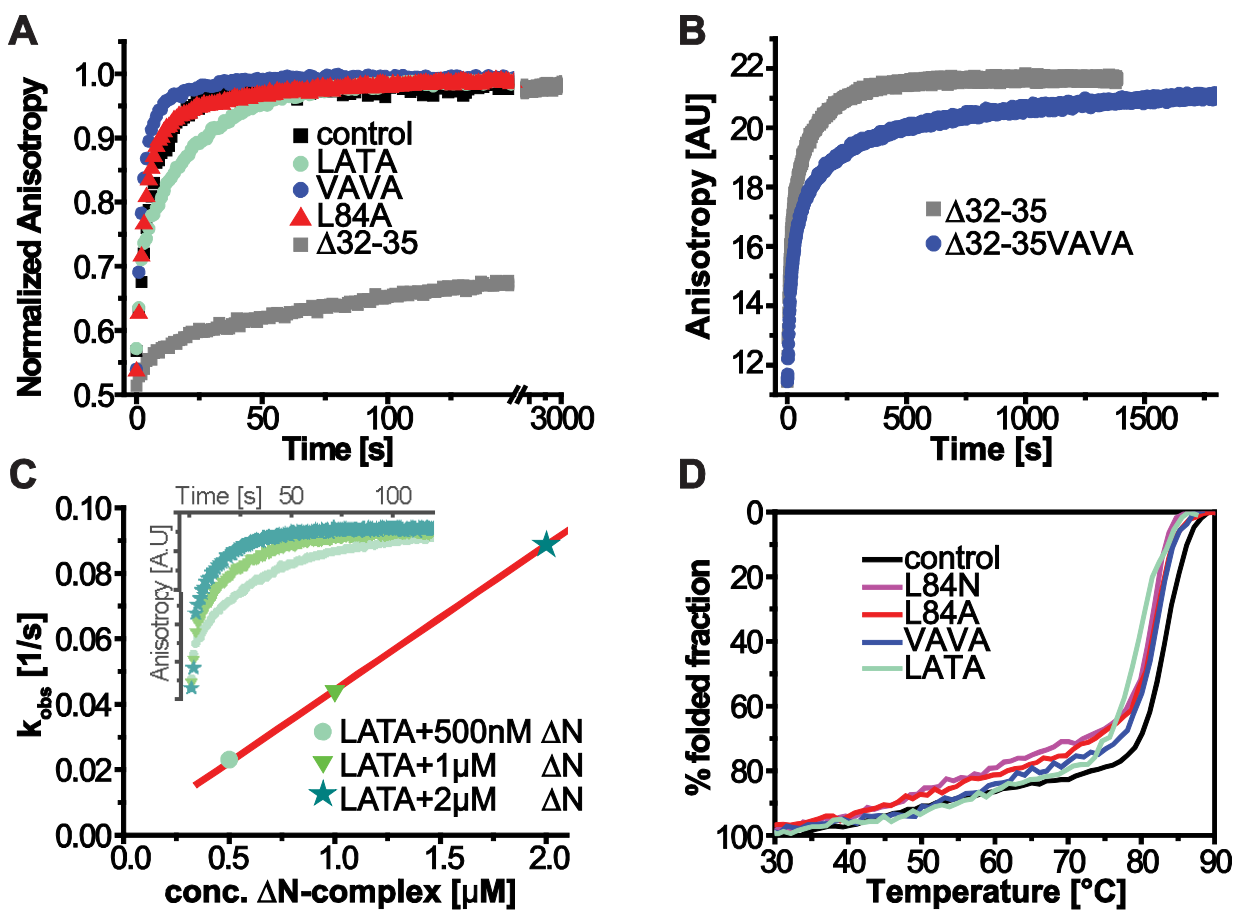

Figure 19. In-vitro binding of syb 2 to a SNAP-25:syntaxin acceptor complex reveals slowdown of kinetics for $\mathbf{N}$ - but not $\mathbf{C}$-terminal mutants. (A) Fluorescence anisotropy measurements of labeled syb 2 mutants and wildtype protein allow characterization of in-vitro binding kinetics. Comparison of two Nterminal mutants (LATA and VAVA) with wildtype syb 2 (control) and a C-terminal mutant (L84A). An Nterminally truncated syb $2(\Delta 32-35$, deletion of amino acids 32 to 35$)$ shows greatly decreased binding 
kinetics (note the axis break) (100 nM Synaptobrevin was added to $500 \mathrm{nM} \Delta \mathrm{N}$-complex). (B) Truncation of the layers -7 and -6 with the VAVA mutation slows binding of syb 2 mutants even more. (C) Determination of rate constants for binding the LATA mutant at different concentrations of the acceptor complex (amount of $\Delta \mathrm{N}$-complex, for an example see insert) allows calculation of the association rate (slope of linear fit, values given in text). (D) C- and N-terminal mutants form stable complexes and maintain similar melting temperatures as revealed by $\mathrm{CD}$ spectroscopic characterization. In-vitro data were obtained by Katrin Wiederhold in collaboration with Dirk Fasshauer, Max-Planck-Institute for Biophysical Chemistry, Department of Neurobiology, Göttingen, Germany.

All mutants used in this study were able to generate stable SNARE complexes with only slight destabilization as revealed by melting curves obtained with $\mathrm{CD}$ spectroscopy (Figure 19C). To characterize the thermodynamic properties of the mutants used in this investigation isothermal calorimetry (ITC) was employed. The "LATA" mutant decreased the affinity of synaptobrevin to SNARE partners from a $\mathrm{K}_{\mathrm{D}}$ of $\sim 2 \mathrm{nM}$ (Wiederhold and Fasshauer, 2009) to $\sim 44 \mathrm{nM}$, whereas the "VAVA"-mutant also in this assay had a milder, but distinguishable, phenotype $\left(\mathrm{K}_{\mathrm{D}} \sim 8 \mathrm{nM}\right.$; Table 1). These data confirm that both N-terminal mutations interfere with the binding of synaptobrevin to the SNAP-25:syntaxin acceptor complex.

Table 1. Thermodynamic properties of $\mathrm{N}$-terminal mutants, determined by isothermal calorimetry (ITC).

\begin{tabular}{|l|c|c|c|c|c|}
\hline $\begin{array}{l}\text { Protein interaction } \\
\text { (Cell + syringe) }\end{array}$ & $\begin{array}{c}\mathbf{K}_{\mathbf{D}} \\
{[\mathbf{n M}]}\end{array}$ & $\begin{array}{c}\Delta \mathbf{H} \\
{[\mathbf{k c a l} / \mathbf{m o l}]}\end{array}$ & $\begin{array}{c}-\mathbf{T} \Delta \mathbf{S} \\
{[\mathbf{k c a l} / \mathbf{m o l}]}\end{array}$ & $\begin{array}{c}\Delta \mathbf{G} \\
{[\mathbf{k c a l} / \mathbf{m o l}]}\end{array}$ & $\mathbf{n}$ \\
\hline$\Delta$ Ncomplex+Syb1-96 & $2.1 \pm 0.6$ & $-29.9 \pm 0.3$ & 18.1 & -11.8 & 1.05 \\
\hline$\Delta$ Ncomplex+SybLATA & $44.1 \pm 12.3$ & $-16.6 \pm 0.4$ & 6.5 & -10.1 & 1.01 \\
\hline$\Delta$ Ncomplex+SybVAVA & $8.4 \pm 2.8$ & $-23.4 \pm 0.4$ & 12.3 & -11.1 & 1.02 \\
\hline
\end{tabular}

Dissociation constant $\left(\mathrm{K}_{\mathrm{D}}\right)$, binding enthalpy $(\Delta \mathrm{H})$, entropy $(\Delta \mathrm{S})$ and free (Gibbs-) energy $(\Delta \mathrm{G})$, binding stoichiometry.

\subsection{The C-terminal SNARE domain of synaptobrevin stabilizes the fusion pore}

\subsubsection{Fusion pore stability is reduced by C-terminal mutations}

Exocytosis of individual vesicles can be studied by means of single spike amperometry (Wightman et al., 1991). Here, release of catecholamines can be detected by a current generated by oxidation of the transmitter at a carbon fiber 
electrode (CFE) placed in close proximity to the cell and held at a potential of $720 \mathrm{mV}$. Typically, individual non-overlapping fusion events can be characterized by a number of parameters describing the geometrical shape of the spike (Figure 21A). In many spikes, initial release of transmitter from a vesicle can be detected in a so-called pre-spike foot, where efflux of the transmitter from the vesicle is already detected, although its diffusion is hindered by the fusion pore (Chow et al., 1992). Subsequent relaxation of the fusion pore allows release of the entire vesicular content thus giving rise to the complete spike.

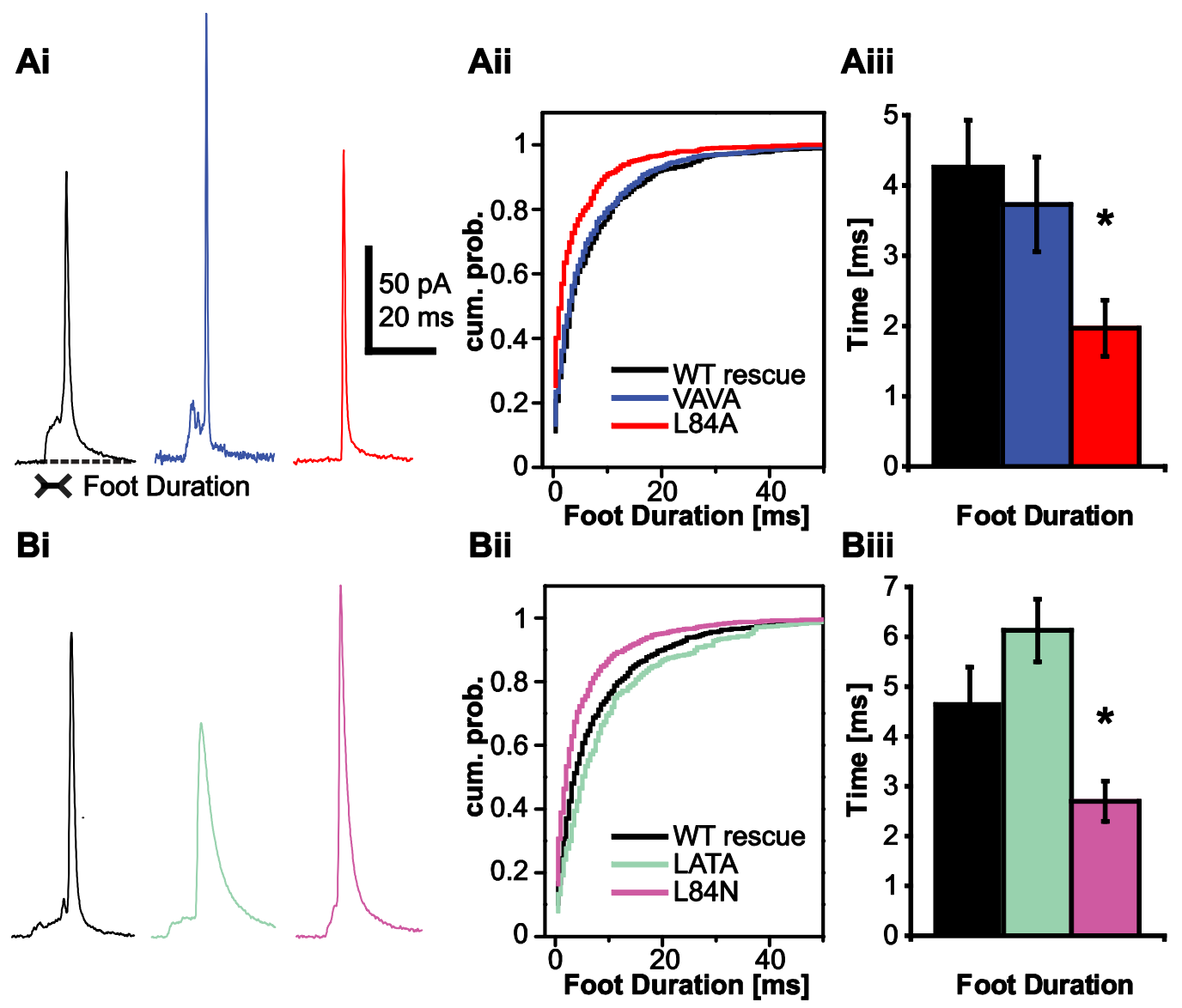

Figure 20. Fusion pore stability is affected by $\mathrm{C}$ - but not $\mathrm{N}$-terminal destabilization of the SNARE complex. (Ai and Bi) Examples of spikes recorded by means of carbon fiber amperometry. (Aii and Bii) Cumulative probability distribution of the pre-spike foot duration shows a shift to shorter feet in C-terminal synaptobrevin mutants (L84A in Aii and L84N in Bii). (Aiii and Biii) The mean foot duration (mean of cell medians) of C-terminal mutants is significantly reduced. (A: WT rescue: $n=16$ cells; VAVA: $n=19$ cells; L84A: $n=19$ cells. B: WT rescue: $n=14$ cells; LATA: $n=15$ cells; L84N: $n=19$ cells. *: $p<0.05$ in oneway ANOVA after log-transformation yielded normally distributed homoscedastic data.) 
In order to elucidate to what extent the weakening of SNARE interaction affects exocytosis on the level of a single vesicle, I infused expressing chromaffin cells with a solution containing $4.5 \mu \mathrm{M} \mathrm{Ca}^{2+}$ and characterized amperometric spikes. I strictly compared groups expressing wildtype protein (WT rescue) with mutants each challenging $\mathrm{N}$ - and C-terminal SNARE interaction. A selective and significant effect was found for $\mathrm{C}$ - but not for N-terminal complex destabilization exclusively reducing the pre-spike foot duration (Figure 20A, B) without affecting properties of the actual spike, such as peak amplitude, charge, rise time, or halfwidth (Figure 21B, C). A reduction in foot duration implies a decreased lifetime of the fusion pore. A change in foot amplitude was not detected (Figure 21Di, Dii). Note that this effect was reproducibly shown in two independent datasets, comparing control to L84A and "VAVA" mutants and comparing control to L84N and "LATA" mutants, respectively (Figure 20A, B; Figure 21A, B). 


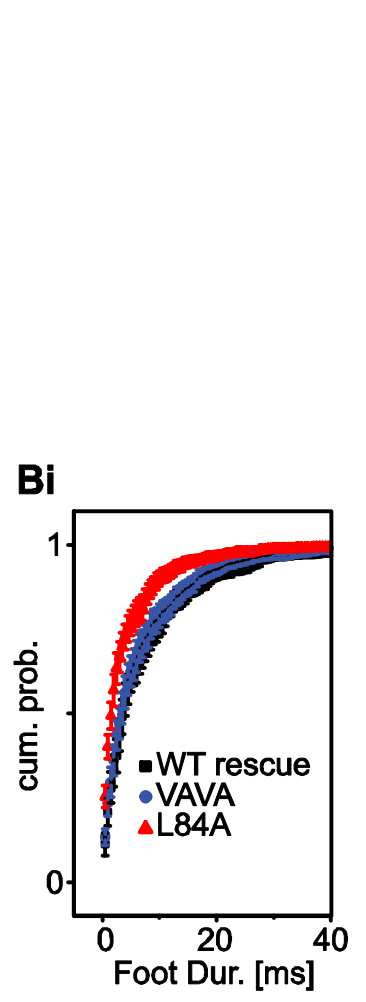

A

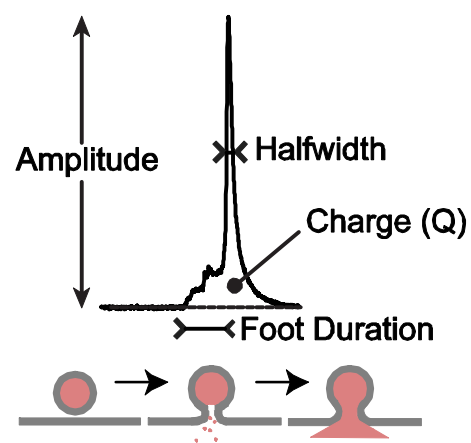

Bi

Bii

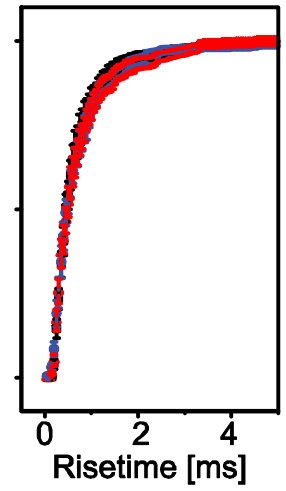

Biv

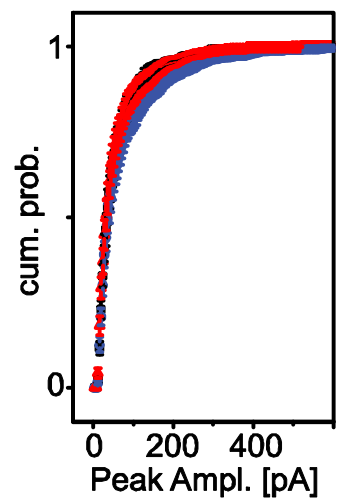

Bv

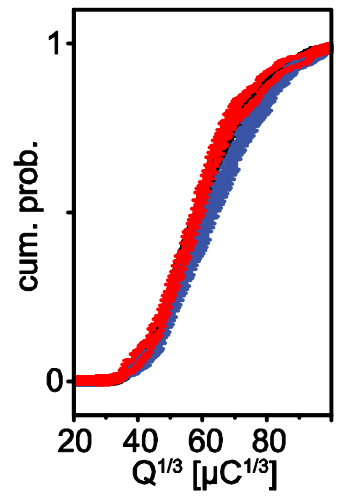

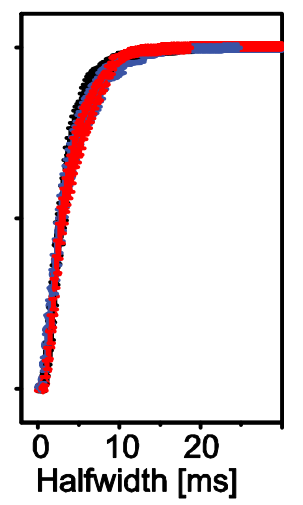

Di

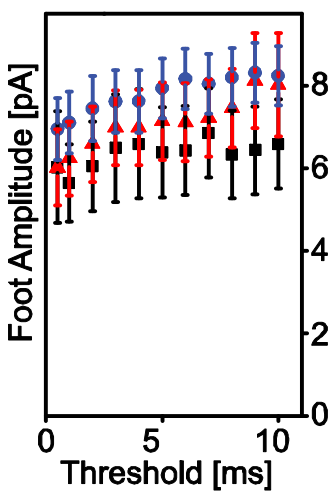

Ci

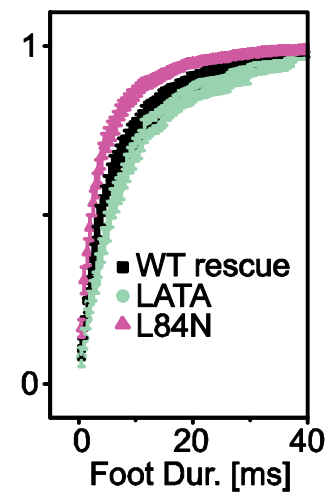

Cii

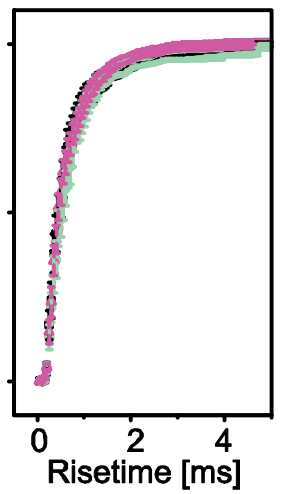

Ciii

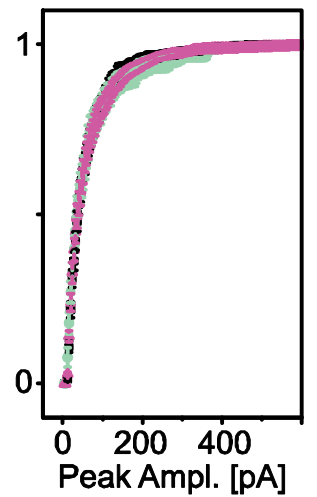

Civ

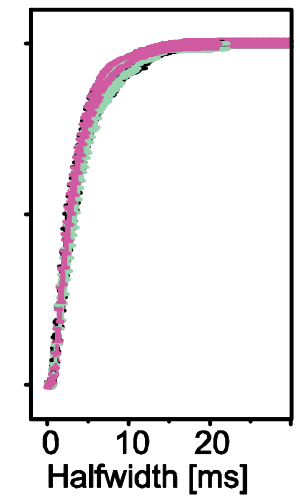

Dii

Cv
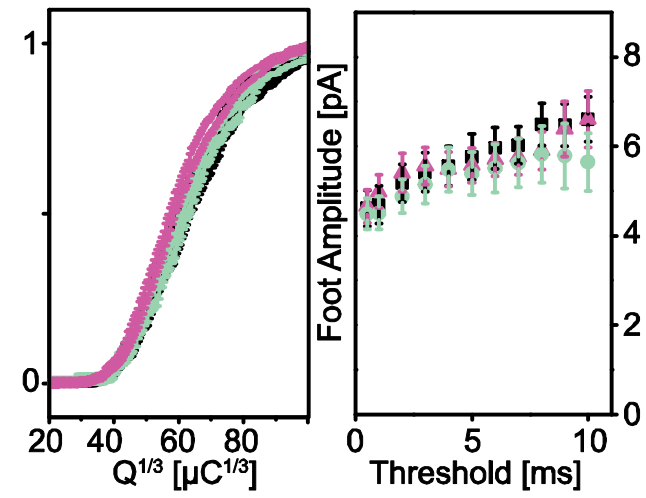

Figure 21. C-terminal mutation selectively affects fusion pore lifetime. (A) Schematic drawing of a spike current recorded by amperometry. The shape of a spike is characterized by its amplitude, its $50-90 \%$ risetime, its halfwidth, its charge $(\mathrm{Q}$, measure of neurotransmitter content in one vesicle) and the cubic root of 
the charge (proportional to the vesicle diameter). Efflux of neurotransmitter from a vesicle through the fusion pore prior to full collapse of the vesicle produces a pre-spike foot. (B) Cumulative probability distribution of amperometric spike parameters, binned data showing mean and SEM of cell distributions. (Bi and Ci) Foot duration. (Bii and Cii) Risetime. (Biii and Ciii) Peak amplitude. (Biv and Civ) Halfwidth. (Bv and Cv) Cubic root of peak charge. (Di and Dii) Amplitude of pre-spike feet as a function of a selected minimal duration (threshold) for feet analyzed. Overlapping values of the pre-spike foot amplitude indicate that fusion pore conductance is unaffected by synaptobrevin mutants and foot shapes are conserved over a broad range of feet length (same cells as in Fig. 20.).

It is interesting that C-terminal SNARE complex destabilization in the two electrophysiological experiments had different effects: in the flash-experiment, secretion was slowed and analyzing pre-spike feet revealed a decrease in fusion pore lifetime. At first glance this is difficult to reconcile: why should an effect, decreasing the rate of fusion in the one experiment speed up the transition from the open state in the other? The rational of the experiments here was to test for a regio-sensitivity and the results clearly show such an effect. However, though not originally the scope of my thesis, investigating fusion pore properties following mutation may add to the understanding of the fusion process. A detailed characterization of fusion pore properties will be presented next.

\subsubsection{The Meyer Jackson proteinaceous pore model}

Meyer Jackson and collaborators suggested the use of a simplified kinetic scheme analogous to the ones used for ion channel gating to analyze the fusion pore duration (Wang et al., 2001). In that scheme (Equation 2), the opening of a fusion pore (from the primed $(\mathrm{P})$ to the open state $(\mathrm{O})$ ) with a rate of $\mathrm{k}_{\mathrm{o}}$ can be followed by either reversible closure $\left(\mathrm{k}_{\mathrm{c}}\right)$ producing a stand-alone foot, SAF, or irreversible pore expansion $\left(\mathrm{k}_{\mathrm{f}}\right)$ leading to full collapse (Wang et al., 2006).

$$
\begin{gathered}
P \underset{k_{c}}{\stackrel{k_{o}}{\rightleftarrows}} O \stackrel{k_{f}}{\rightarrow} F \\
\tau_{o}=\frac{1}{k_{f}+k_{c}}
\end{gathered}
$$

Within this framework, a decreased lifetime of the fusion pore $\left(\tau_{0}\right.$, Equation 24$)$ could be due to an increased rate of expansion, an increased rate of re-closure, or 
both. These possibilities, in theory, can be distinguished by analyzing the fraction of standalone feet (SAF), which should increase in the first case and decrease in the second case.

The detection and correct identification of SAF are difficult. This is a problem arising from the experimental design, where secretory events in carbon fiber amperometry can only be resolved adequately when occurring very close to the detection electrode. However, secretion is not limited to the area in contact with the recording electrode, but actually occurs all over the cell. Events arising at a distance will lose some of their information: in terms of temporal accuracy due to diffusion and in terms of signal amplitude due to the fact that only a fraction of the transmitter will be detected (Sakmann and Neher, 1995). For typical amperometric spike analysis these difficulties are usually circumvented by only considering signals exceeding a certain amplitude. The identification of SAF is complicated since it is currently not clear what a SAF would exactly look like in an amperometric experiment. One would assume that an SAF should look like an isolated pre-spike foot and it has been suggested that these events are characterized by their rectangular shape (Cai et al., 2008; Wang et al., 2006; Zhang and Jackson, 2008). Still, it is difficult to clearly distinguish between standalone feet that occur close to the recording electrode and signals originating from spikes far away. Both signals will be small in amplitude and although spikes from fusion events far away will be broad, these signals most likely will look more or less symmetrical.

Using two different criteria for the identification of SAFs, one based on a more rectangular shape expected for these events, and one based on their lower amplitude, no changes in the fraction of SAFs upon C-terminal mutation could be detected (Figure 22). 
Ai

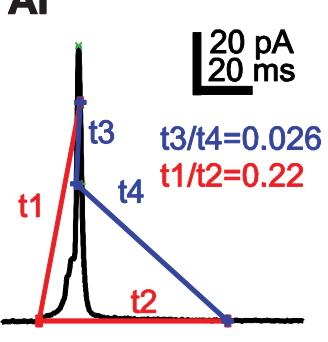

C

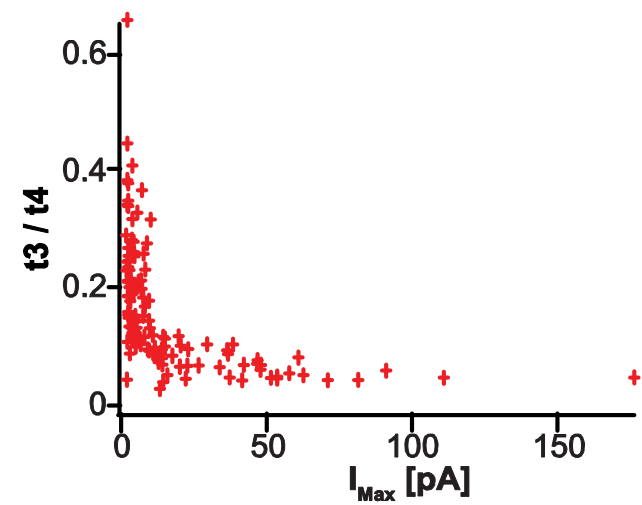

Aii

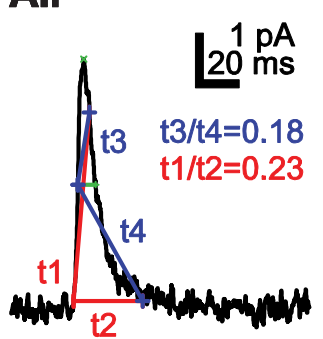

B

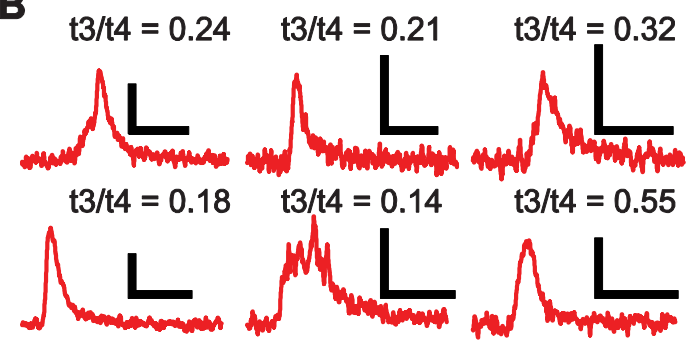

D

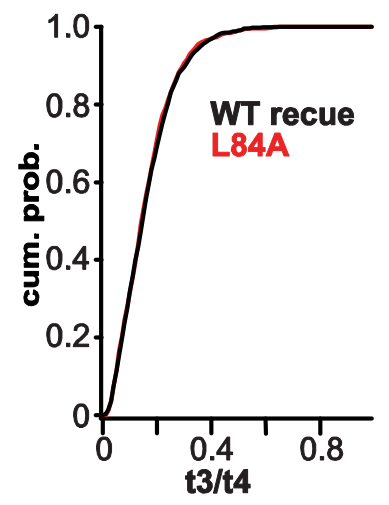

E

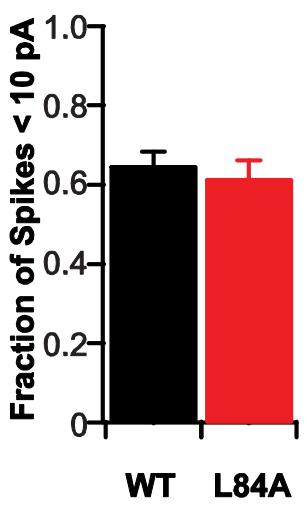

Figure 22. Attempt to detect stand-alone-feet (SAF) in embryonic mouse chromaffin cells. (A) Ai and Aii show two spikes, a large one with a prominent prespike foot (Ai) and a smaller one (Aii). Two criteria were applied: $\mathrm{t} 1$ and $\mathrm{t} 2$ are two different duration measures, both starting at the point where the signal departs by more than 1xRMS (root-mean-square) of the noise above baseline. The end point of $\mathrm{t} 1$ was defined as the mean spike amplitude between the two time boundaries defined by the half-way points between peak amplitude and baseline. The end point of $\mathrm{t} 2$ is defined as the time when the signal returned to within 1xRMS of the baseline. These definitions were taken from (Wang et al., 2006), where the quotient $t 1 / t 2$ was proposed as a useful criterion for detecting SAFs. However, in this case the two spikes (Ai and Aii) result in rather similar $\mathrm{t} 1 / \mathrm{t} 2$ quotients, in spite of very dissimilar amplitudes and shapes. The reason is that the large spike in Ai has a rather long prespike foot, which enters into the calculation of $t 1$. Therefore two new duration measures were defined: $\mathrm{t} 3$, and $\mathrm{t} 4$, whose end-points are identical to those of $\mathrm{t} 1$ and $\mathrm{t} 2$, but with the common start point at the time the spike exceeds half its maximal amplitude. Since most pre-spike feet were smaller than half of the maximal amplitude, the $\mathrm{t} 3 / \mathrm{t} 4$ quotient might be a better measure for the shape of the spike itself, when pre-spike feet are of longer duration. (B) A collection of small events, with their t3/t4 quotient. For most of the events it is hard to determine off-hand whether they are SAFs, or just small spikes originating far away from the recording electrode. The exception is the second-to-last event, which can hardly be interpreted as anything but an SAF. However, such events were relatively rare; most were more transient. (C) Plot showing the dependency of the $\mathrm{t} 3 / \mathrm{t} 4$ quotient on the maximal peak amplitude of the event for all events detected from a single cell. The plot clearly shows that the shape of events $<10 \mathrm{pA}$ is more rectangular ( $\mathrm{t} 3 / \mathrm{t} 4$ larger) than larger events. (D) Cumulative probability distribution of the $\mathrm{t} 3 / \mathrm{t} 4$ criterion is unchanged in the L84A mutant compared to wildtype rescue. (E) The fraction of events with amplitude $<10 \mathrm{pA}$ was unchanged in the L84A mutant compared to wildtype rescue (cell means, WT rescue $n=11$, L84A expressing cells $n=12$ ).

This indicates that either most events detected were not SAFs, that the changes seen are not caused by modification of a single transition rate in an otherwise unchanged kinetic scheme or that the model itself is inaccurate. A closer look at this model and the implications for vesicle pool kinetics will be addressed next. 


\subsubsection{From single spikes to pool kinetics}

The analysis of signals that may be attributed to SAF did not succeed in providing a sensible link between the parameters of the fusion pore obtained by single spike analysis and pool kinetics measured in the flash-experiment. In order to evaluate the proteinaceaus pore model more thoroughly its inferences for pool kinetics were considered mathematically. The simple reversible pore formation model above allows for a direct calculation of the predicted capacitance response based on the kinetic model stated above. Solving the set of differential equations (see Materials and Methods for detailed delineation [4.7]) yields the following expression for the capacitance response:

$$
\Delta C_{M}=P_{0}\left(1-\frac{k_{o}+\lambda_{b}}{\lambda_{b}-\lambda_{a}} e^{\lambda_{a} t}-\frac{k_{o}+\lambda_{a}}{\lambda_{a}-\lambda_{b}} e^{\lambda_{b} t}\right)
$$

With

$$
\begin{aligned}
& \text { (8) } \lambda_{a}=-\frac{k_{o}+k_{c}+k_{f}}{2}+\sqrt{\frac{\left(k_{o}+k_{c}+k_{f}\right)^{2}}{4}-k_{o} k_{f}} \\
& \text { (9) } \lambda_{b}=-\frac{k_{o}+k_{c}+k_{f}}{2}-\sqrt{\frac{\left(k_{o}+k_{c}+k_{f}\right)^{2}}{4}-k_{o} k_{f}}
\end{aligned}
$$

Although the above equation in its form agrees well with the classical two pool model (Voets, 2000) as it contains two exponential terms, this model fails to describe the observed capacitance responses for the following reasons: $\lambda_{a}$ and $\lambda_{b}$ here resemble time constants of two different components (one fast, described by $\lambda_{\mathrm{b}}$ and one slower component, described by $\lambda_{\mathrm{a}}$ ). Additionally, in this model, the rate constants $\mathrm{k}_{\mathrm{c}}$ and $\mathrm{k}_{\mathrm{f}}$ determine the lifetime of the fusion pore $\left(\tau_{\mathrm{o}}\right)$. Thus, these values can now be compared (see Materials and Methods for a concise delineation [4.7.1]), allowing inferences from the single spike experiments for whole cell capacitance measurements. For instance, it can be shown that the fastest phase of the capacitance response (given by the second eigenvalue, $\lambda_{b}$ ) should be as fast as or faster than the mean lifetime of the fusion pore:

$$
\text { (26) }-\lambda_{b} \geq \frac{1}{\tau_{o}}
$$


This would correspond to a fast time constant of less than $\sim 5 \mathrm{~ms}$, which is the mean pre-spike foot duration under the conditions tested. Such a fast component, however, is not observed in flash-evoked capacitance responses. Usually the value of the RRP is in the range of $\sim 20 \mathrm{~ms}$. The above scheme can therefore only explain pool kinetics if the amplitude of the exponential term containing $\lambda_{b}$ is essentially zero. It can be shown that this (see Materials and Methods for a concise delineation [4.7.1]) is only the case if $\mathrm{k}_{\mathrm{c}}$ is zero, which in actual fact implies that under the conditions tested here fusion pore formation is an essentially irreversible process.

\subsubsection{Towards concluding the pore}

The findings presented here argue against a fusion pore lined by proteins and a mode of transmitter release predominantly featuring pore re-closure (kiss and run); an extension of this model to pool kinetics fails in the prediction of multiexponential capacitance behavior. Slowing of pool kinetics cannot be explained by an increase in the rate favoring pore re-closure since no increase in signals attributed to standalone feet was found. Furthermore, considering the above calculations the rate of fusion pore closure must be next to zero in order to account for the observations in the flash experiment. An increase in the rate favoring pore expansion $\left(\mathrm{k}_{\mathrm{f}}\right)$ would be in line with a decrease in pre-spike foot duration observed, but would not lead to decreased pool kinetics. This suggests that the process of pore expansion and the increase of cellular capacitance may be less tightly coupled. A model considering this idea will be discussed later.

\subsection{The main findings in a nutshell}

The current findings allow the deduction of mechanisms involved in neurotransmitter release. The study of morphology and physiology of release in mutant mouse chromaffin cells allow the dissection of individual reactions involved in docking, priming and exocytosis triggering. 
The main findings are the following:

\subsubsection{The SNARE proteins and their binding partners act in docking}

- SNAP-25 and Munc-18 are essential docking factors acting in synergy.

- Overexpression of SNAP-25, or expression of a C-, but not N-terminal syb 2 fragment in the Munc-18 null background restores docking without restoring fusion.

- Munc-18 has a role upstream and downstream of docking.

- Synaptotagmin 1 acts as the vesicular docking partner.

- Synaptotagmin promotes docking by binding to SNAP-25 in a syntaxin:SNAP-25 acceptor complex, possibly stabilized in 1:1 stoichometry by Munc-18.

- Docking and priming are coupled, but distinguishable reactions.

\subsection{2 $\mathrm{N}$ - to C-terminal SNARE zippering drives priming and fusion}

- N-terminal SNARE destabilization decreases the priming rate.

- N-terminal synaptobrevin destabilization selectively decreases the rate and affinity of SNARE complex formation in-vitro.

- Layers +4 and +6 of the SNARE complex are essential for transmitter release.

- C-terminal SNARE complex assembly is important for rapid release and exocytosis triggering.

- The fusion pore is stabilized by the C-terminus of the SNARE complex.

- Fusion pore formation in the flash experiment is an essentially irreversible process. 


\section{Discussion}

The work presented here allows inferences on the role of the SNARE proteins and binding partners at the different stages of the vesicular release cycle, specifically addressing the reactions involved in vesicle docking, priming and exocytosis triggering.

\subsection{Docking}

\subsubsection{The role of the SNARE proteins in docking}

In experiments addressing the role of the SNAREs in vesicle docking there was collaboration with Prof Matthijs Verhage's laboratory where Heidi de Wit performed the ultrastructural analysis of chromaffin cells. These were complemented by electrophysiological measurements of secretion in flash-evoked release. It is particularly interesting that phenotypes in docking do show effects on physiological behavior. For instance, the decrease of the sustained component by overexpression of the syt $1 \mathrm{C} 2 \mathrm{AB}$ domain agrees well with effects decreasing the rate of vesicle maturation thus suggesting that the reactions of docking and priming are closely coupled. On the other hand, it is striking that manipulations capable of rescuing docking in the absence of Munc-18 are incapable of restoring secretion, which implies a post-docking role of Munc-18. In previous studies it was suggested that Munc-18 bound to closed syntaxin acts in docking (GulyasKovacs et al., 2007; Voets et al., 2001b), whereas an interaction with the SNAREcomplex is required for vesicle priming (Deak et al., 2009). Conversely it was shown that synaptic vesicle docking in the nematode C.elegans requires syntaxin in the open conformation (Hammarlund et al., 2007). 
The work presented here allows one to surmise that the involvement of Munc-18 in the promotion of docking may be due to a necessary stabilization of a 1:1 SNAP-25:syntaxin acceptor complex preventing the formation of an unproductive 2:1 complex. This is similar to the situation in-vitro (Pobbati et al., 2006; Zilly et al., 2006). It was recently shown that Munc-18 indeed stabilizes a 1:1 complex invitro (Weninger et al., 2008). Although to date no evidence exists that a similar off-pathway may be present in vivo, two observations strengthen this hypothesis: firstly, overexpression of SNAP-25 at high levels did restore docking in the absence of Munc-18 possibly by increasing the concentration of the 1:1 complex by law of mass action. Secondly, a soluble C-terminal fragment of synaptobrevin shown to act via stabilization of the 1:1 complex in-vitro, had a similar effect. The fact that docking was not restored by expression of an N-terminal syb 2 fragment does not necessarily imply that synaptobrevin interaction is necessary for docking. An N-terminal fragment most likely only induces $\alpha$-helicality at its binding site, whereas the C-terminal fragment renders the entire complex $\alpha$-helical (Pobbati et al., 2006) and this may be required for syt 1 interaction.

In an unproductive 2:1 syntaxin:SNAP-25 acceptor complex, synaptotagmin may not be able to bind to SNAP-25 and may therefore fail in promoting docking. The binding sites have been implicated at residues 51, 52 and 55 of SNAP-25 (Rickman et al., 2006) and the second syntaxin may shield a possible interaction of the syt $1 \mathrm{C} 2 \mathrm{AB}$ domain. This is different from the situation of synaptobrevin binding as it was shown that syt 1 and syb 2 do not compete for SNARE binding in-vitro (Rickman et al., 2006).

The most important findings for the understanding of the process of vesicle docking are the identification of two novel docking factors, SNAP-25 and synaptotagmin 1. Although it could now be established that the absence of SNAP25 has a severe effect on vesicle docking, these results are in stark contrast to results published previously (Sorensen et al., 2003b). These experiments were not performed as part of this thesis. Therefore, for a discussion of the reasons that may explain these discrepancies, please refer to de Wit et al. (2009). 
The discovery of the vesicular docking factor syt 1 is of particular interest. Although synaptotagmin 1 has been generally considered to be the prime $\mathrm{Ca}^{2+}$ sensor for fast release (Geppert et al., 1994) this raises the interesting issue of additional actions of synaptotagmin, possibly contributing to the speed-up of release by targeted association to $\mathrm{Ca}^{2+}$ hotspots in the cell (Neher and Penner, 1994).

\subsubsection{A model of the docking reaction in chromaffin cells}

Employing the four genes identified in docking, a minimal working model for docking can now be synthesized (Figure 9). In a first reaction Munc-18 binds to syntaxin 1 in its closed conformation. Then SNAP-25 joins, thus forming a syntaxin:SNAP-25 1:1 acceptor complex stabilized by Munc-18. Thirdly, secretory vesicles become docked when synaptotagmin 1 binds to the acceptor complex. Munc-18 plays an additional role in the downstream reaction of priming, possibly by facilitating the binding of synaptobrevin 2 .

\subsubsection{The experiments on a conceptual level}

In the current study docking was assessed by ultrastructural analysis of embryonic chromaffin cells supplemented with physiological measurements of secretion. A general concern in the assessment of docking is the problem of fixation necessary to preserve ultrastructural features of the specimen. The reagents used for this purpose are harsh and toxic chemicals, putatively inducing artifacts, as discussed by Hammerlund and colleagues (2007). It was indeed observed, that when fixing whole chromaffin glands a reduced number of docked vesicles was found. Under these conditions, slow diffusion of fixatives into the tissue may be a problem, since they may activate secretion (de Wit et al., 2009). This effect could be circumvented by application of the high pressure rapid freeze (HPF) technology, generally thought to be prone to fewer artifacts (Hammarlund et al., 2007). For this reason docking in SNAP-25 deficient mice was also assessed following HPF fixation and the defect was also confirmed here (de Wit et al., 2009). 
Another specific feature of data obtained after fixation is the fact that observations are restricted to a "snapshot" of an originally dynamic process. Unlike physiological measurements that can be performed at high temporal resolution and allow inference of kinetic changes, observations after fixation are limited to the inspection of reactions frozen in a steady state. In this sense it is not possible to determine precisely the individual reactions affected by mutation, but merely shifts of equilibrium concentrations in an unknown kinetic scheme. One can suppose that syt 1 docks vesicles by actively initiating the attachment of the vesicle to the plasma membrane. However, equally valid explanations for the reduced number of docked vesicles in the syt 1 knockout are possible. For instance, it is conceivable that syt 1 indeed plays a crucial role in the stable association of secretory vesicles to the plasma membrane, but by disfavoring undocking rather than promoting initiation of docking. A distinction between these two possibilities could in theory be tested by employing physiological measurements like total internal reflection fluorescence microscopy (TIRFM). This would specifically allow the determination of the residence time and force exerted on the secretory vesicles in docking (Toonen et al., 2006). However, application of this technique is complicated by the uncertain assertion of docked vesicles due to (1) the inability to calculate the exact distance to the plasma membrane, (2) the problem of normalization since not all vesicles will be visible in the TIRF plane, (3) the bleaching of fluorescent dyes and finally (4) the problem that not all but only a subpopulation of vesicles can be labeled (Verhage and Sorensen, 2008). Despite these obstacles, when performed these experiments will greatly add to the understanding of the role of synaptotagmin in docking.

\subsubsection{Distinguishing docking and priming}

A number of schemes are conceivable that describe the manner in which docking and priming are coupled. All models assume that primed vesicles are docked but the mechanism, especially the number of steps that promote this action, differs. 
Firstly (1), one could imagine that a single docking reaction is an essential prerequisite for all downstream reactions of priming and triggering of secretory vesicles. In this sense docking and priming would be linearly coupled reactions contributing to the maturation of vesicles. This corresponds to the model depicted in Figure 2 and is presented in Rettig and Neher (2002).

Secondly (2), a refined version of this model includes the possibility that not all vesicles found docked actually are capable of maturing to the primed state but are docked via a reaction that does not allow further transition to releasable states ("dead-end" docking) (Verhage and Sorensen, 2008). According to this scenario, priming also requires prior docking of vesicles. Yet, additional parallel pathways exist that allow for docking but not priming of vesicles. This may explain the fact that docked vesicles even remain when primed vesicles are depleted (Sudhof, 1995). However, both definitions imply a linear coupling of docking and priming, suggesting that defects in functional docking should also have similar defects in priming.

Alternatively (3), one could imagine that docking and priming actually are morphological and physiological correlates of the same process (Siksou et al., 2009). If this was the only docking reaction it would be difficult to explain why the number of vesicles docked greatly exceeds the number of vesicles primed (Rettig and Neher, 2002). Nevertheless, if parallel pathways for docking existed, one that promoted a combined docking/priming reaction and at least one that promoted docking without direct priming, this model could account for the observations. It should be noted that this model would imply that defects on priming should show defects in docking.

In this sense, both the linear sequential coupling of these sates (1 and 2) as well as the scheme suggesting a single docking/priming reaction (3) imply similar effects if docking was impaired: in both cases a correlation between a defect in docking and priming should exist. The distinction is whether these are governed by the same or sequential reactions. A distinction between these possibilities could be provided by experiments showing that docking is normal in absence of priming, 
favoring the idea of a sequential coupling, or by experiments that reveal a defect on docking without affecting priming.

Expression of the syt $1 \mathrm{C} 2 \mathrm{AB}$ domain on a wildtype background greatly reduced docking, but the effect on secretion was subtle. A reduction of the sustained rate of exocytosis appears to be a fairly weak effect compared to the harsh defect in docking especially as the size of the burst component, which includes the readily releasable pool, was not changed. This may favor the existence of parallel pathways for docking and suggests that only a small fraction of vesicles normally found docked can account for release. The fraction of vesicles that remained docked in these experiments is roughly $1 / 3$ of the ones found under control conditions and corresponds well to the fraction of docked vesicles typically primed (Rettig and Neher, 2002). This implies that most of the vesicles seen docked were primed and therefore may favor the idea of a single docking/priming reaction under these conditions. However, one could also imagine a sequential process with a reversible reaction strongly favoring the primed state in equilibrium.

Although a single docking/priming reaction may explain above observations, the refined definition of the primed state as presented here strongly disfavors this idea: it was shown that vesicle priming corresponds to an N-terminal assembly of synaptobrevin to SNARE partners. Previous experiments have suggested that synaptobrevin is not involved in docking (Borisovska et al., 2005). This implies an additional priming step, where synaptobrevin joins the syntaxin:SNAP-25 complex. Also, a heterogeneity of the docked state is established, which may reflect different maturation states: it was found that only the most strongly attached and least mobile vesicles are primed (Nofal et al., 2007; Toonen et al., 2006). However, the current understanding that synaptobrevin is not involved in docking should be carefully validated to exclude the possibility that due to nonideal experimental conditions a docking defect was missed, as was previously the case for SNAP-25 (Sorensen et al., 2003b; de Wit et al., 2009).

In the absence of Munc-18 docking could be restored by overexpression of SNAP-25 or a C-terminal synaptobrevin fragment. This restoration of docking 
occurred in the absence of secretion also arguing for sequential coupling of docking and priming reactions.

\subsection{Conclusion}

In conclusion it can be maintained that the experiments presented here contribute significantly to the current understanding of the docking process by the characterization of the sequence of reactions driving docking and by the identification of the molecular factors involved therein. It is especially interesting that the experiments presented here demonstrate that docking and priming are distinguishable reactions: although it is possible to restore docking in the absence of Munc-18 by stabilizing a syntaxin :SNAP-25acceptor complex, secretion under these conditions is deficient. On the other hand, in experiments in which the soluble $\mathrm{C} 2 \mathrm{AB}$ domain of synaptotagmin was expressed in a wildtype background, a correlation exists between a defect in docking and a defect in secretion, suggesting that these reactions are closely coupled. 


\subsection{Sequential SNARE assembly comprises priming and triggering}

Using mutagenesis studies the hypothesis of sequential SNARE complex assembly for priming and fusion of secretory vesicles was tested

- on the level of distinct kinetic pools by characterizing release in flashevoked exocytosis,

- on the level of the single vesicle by means of single spike amperometry and

- on the level of the isolated proteins by fluorescence anisotropy and ITC experiments.

The observed regio-sensitivity of SNARE interaction augments the hypothesis of sequential N- to C-terminal SNARE complex assembly with a partly assembled complex corresponding to the primed vesicle state.

Previous studies in SNAP-25 using mutagenesis have already suggested a regiosensitivity to SNARE complex destabilization and have implied a two-step assembly with distinct steps reflecting priming and triggering in exocytosis (Sorensen et al., 2006). However, SNAP-25 is also important for the first step in SNARE-complex assembly: the formation of the SNAP-25:syntaxin acceptor complex (Fasshauer, 2003). In this sense it is not clear whether mutations in SNAP-25 merely affected the final formation of the SNARE complex itself or also interfered with the generation of the SNAP-25:syntaxin acceptor complex. In contrast, the binding of syb 2 to the acceptor complex occurs downstream, constitutes the addition of the vesicular SNARE to the complex and is therefore intimately linked to actual fusion.

In addition, the importance of SNAP-25 in the docking of secretory vesicles (see above, de Wit et al., 2009) further complicates interpretation of these data with regard to downstream processes. In the case of syb 2 no similar effect has been reported and studies addressing morphology of knockout cells have not reported any effect on vesicle docking (Borisovska et al., 2005; Gerber et al., 2008). 
Hence utilizing syb 2 mutants allows a clean dissection of vesicle priming and exocytosis triggering and thus augments previous findings in SNAP-25. Moreover, the mutations used are milder and, in addition show clearer phenotypes. Indeed, the mutants of synaptobrevin used here allowed an almost binary interpretation of the involvement of $\mathrm{N}$ - and $\mathrm{C}$-terminal regions of the SNARE complex with regard to priming and fusion respectively. For instance, the very mild N-terminal double point mutations in the "LATA" and "VAVA" mutant showed a phenotype with a selective effect on the amplitude but identical kinetics of release. Here reduction of pools exactly mirrored a decrease in the sustained rate and made a strong case for a selective decrease in the rate of priming in an otherwise unchanged kinetic scheme (Voets, 2000; Voets et al., 1999).

In contrast, mutations in layers -7 and -6 of SNAP-25 did not result in significant changes of exocytosis (Sorensen et al., 2006). One reason explaining this discrepancy may be the difference in binding mode: whereas SNAP-25 engages in a complex with syntaxin prior to the binding of synaptobrevin, synaptobrevin joins in isolation and concludes SNARE complex assembly. In this sense, the mutagenesis experiments in SNAP-25 examines the binding of synaptobrevin to SNAP-25 and syntaxin 1. Particularly in layers -7 and -6 the interaction of synaptobrevin with syntaxin may suffice for stable complex formation, since syntaxin greatly contributes to the stability of hydrophobic interaction by adding two very hydrophobic residues (Kyte and Doolittle, 1982): an isoleucine in layer 7 and a leucine in layer -6 . On the other hand, mutation of the layer -7 residue (threonine) at position 29 to an alanine on the first SNAP-25 alpha helix in the previous study (Sorensen et al., 2006) could not have greatly reduced the hydrophobic interaction, since it replaced a hydrophilic residue with a hydrophobic one. Replacement of a methionine with an alanine in layer -6 exchanged two residues of similar hydrophobicity. Therefore it may well be that the previous study did not succeed in the appreciation of N-terminal SNARE interaction necessary for priming because the destabilization introduced by the mutation was too weak and compensated by syntaxin interaction. 
Similar effects as in the "LATA" and "VAVA" mutants were detected closer to the core of the complex, in layers $-1,0,+1$ and +2 although a deviation of poolsize reduction and sustained rate decrease might imply that the resulting effects of SNAP-25 destabilization at these positions changed the kinetic scheme in a more complex way (Sorensen et al., 2006).

However, it may well be that the initial association of synaptobrevin to SNARE partners occurs more closely to the centre of the SNARE motif. Indeed, the data obtained following mutation of synaptobrevin in layer -3 suggests this. Here the amplitude of the response was reduced even more than in the "VAVA" or "LATA" mutants. Similarly here effects on the rate of fusion were not observed although this is more difficult to assess due to the small amplitude of the signal. In this sense, the most N-terminal region of the SNARE motif may not be essential for the initiation of binding, but surely for the stabilization of the partly assembled complex. Perhaps complex nucleation occurs more closely towards the core of the SNARE motif.

Mutations in layers +4 and +6 had a severe effect on secretion. Surprisingly, the layer +4 mutant $(\mathrm{L} 70 \mathrm{~A})$ did not act as a dominant negative when expressed in wildtype cells. This is different to the situation of a double mutant in SNAP-25 in layer +5 (both mutations to alanines) where expression of the mutant in wildtype cells greatly reduced secretion, even below the level of secretion in the SNAP-25 knockout (Sorensen et al., 2006). The fact that the L70A mutant did not act as a dominant negative regulator of exocytosis may imply that this mutant is not correctly localized, fails in stable formation of SNARE complexes or is unable to compete with wildtype syb 2 although strongly overexpressed. Interestingly, the remaining secretion in the $\mathrm{L} 70 \mathrm{~A}$ mutant seems to follow the kinetics of the wildtype situation, whereas secretion is kinetically compromised in the layer +6 mutant (F77A). This may imply that few, possibly only the three most C-terminal layers of the SNARE complex, are involved in the conformational change directly driving fusion when exocytosis is triggered. Most of the SNARE motif, at least until layer +4 is therefore involved in priming the vesicle, in complete agreement with the previous study in SNAP-25 (Sorensen et al., 2006). 
In the current study destabilization of C-terminal interaction by a single point mutation in layer +8 in syb 2 already displayed a phenotype that very closely resembled the double and triple mutants of layers +8 (double point mutant) and +8 and +7 (triple point mutant, 2x layer $+8,1 \mathrm{x}$ layer +7 ) in SNAP-25 (Sorensen et al., 2006).

Together, these data strongly support the conclusion of the previous work, but substantiate the experimental data utilizing the vesicular SNARE protein syb 2. Also, in the current work, the effect of SNARE complex destabilization was assessed on the level of the single vesicle by carbon fiber amperometry and on isolated protein interaction by fluorescence anisotropy and ITC.

\subsubsection{Biophysical data}

The kinetic data obtained from fluorescence anisotropy measurements showed a slowdown of SNARE complex formation for N- but not C-terminal destabilization suggesting that the initial binding of synaptobrevin to the SNAP-25:syntaxin complex is mediated via its $\mathrm{N}$-terminus in agreement with previous findings (Pobbati et al., 2006). This is also in line with the "LATA" and "VAVA" mutant showing a reduced affinity to the acceptor complex, as revealed by ITC experiments.

The fact that fluorescence anisotropy measurements initially failed to reveal an effect on binding kinetics in the "VAVA" mutant, in spite of its strong effect in vivo may be due to the fact that the reaction in vivo may be catalyzed by additional factors, possibly Munc-18, increasing the rate of vesicle priming. Nterminal truncation of syb 2 greatly decreased the rate of complex assembly (Figure 19B), once again stressing the need for N-terminal interaction for initial binding of syb 2 to the acceptor complex. After truncation binding was further slowed by the introduction of the "VAVA" mutant, substantiating the need for interaction of layers -5 and -4 for complex initiation in-vitro and in vivo. 


\subsection{The SNAREs and the fusion pore}

\subsubsection{The C-terminal SNARE motif adds to the stability of the fusion pore}

By means of carbon fiber amperometry the effect of regional SNARE complex destabilization on the fusion of individual vesicles could be described. In a thorough analysis of the geometrical properties of single spikes recorded from cells expressing the various constructs a selective and significant effect on the prespike foot duration for $\mathrm{C}$ - but not $\mathrm{N}$-terminal SNARE complex destabilization was found. C-terminal mutation decreased the lifetime of the fusion pore. This is different from the increase of pre-spike foot duration observed in the absence of syb 2 (Borisovska et al., 2005) and augments the hypothesis of sequential $\mathrm{N}$ - to Cterminal SNARE complex assembly, supporting the idea that C-terminal assembly is most closely coupled to exocytosis triggering.

These results are poles apart from a study performed in PC-12 cells (Han and Jackson, 2006), where it was found that mutations in N-terminal layers -7 and -6 to alanines (corresponding to the "LATA" mutant) showed an increase in the prespike foot duration. This may be due to the different preparations (PC-12 cells vs. embryonic chromaffin cells), different stimulation procedures (stimulation with high $\mathrm{K}^{+}$vs. infusion with $\mathrm{Ca}^{2+}$ ) or different statistical analysis (comparing spike means of all spikes vs. comparing means of cell medians). A further important difference is the overexpression of the mutant constructs on the wildtype background vs. mutant overexpression in a knockout background as presented here. This effect becomes immediately clear when comparing the studies performed using a mutant bearing a mutation in layer +4 of syb 2 (L70A) which was reported to show an increase in pre-spike foot duration in PC-12 cells (Han and Jackson, 2006) but in the investigation here only led to marginal rescue secretion in double knockout cells and revealed no effect when expressed on a wildtype background. This suggests that this mutant cannot participate in ordinary fusion. These discrepancies are contradictory and demonstrate the necessity of a clean genetic background for mutagenesis studies. These may generally be much more tedious but avoid the pitfalls of interpretation of data that are a superposition 
of effects arising from the activity of mutants with the background activity of endogenous proteins.

\subsubsection{From single channels to pre-spike feet?}

Spikes from cells expressing syb 2 bearing a point mutation in layer +8 had shorter feet. A decrease of the pre-spike foot duration is thought to reflect a decrease in fusion pore lifetime and thus fusion pore stability. A model by Meyer Jackson and colleagues attempts to describe properties of the fusion pore by a kinetic scheme (Equation 2) very similar to the ones applied to single channel gating (Wang et al., 2001). This model implies that the fusion pore forms reversibly, upon re-closure producing standalone feet in a "kiss-and-run"-like mechanism of fusion (Ceccarelli et al., 1973). Although the existence and physiological relevance of "kiss-and-run" in synaptic exocytosis is under heavy debate (He and Wu, 2007; Rizzoli and Jahn, 2007), this mechanism has been thoroughly described in chromaffin cells by means of patch amperometry (Ales et al., 1999). Therefore, the idea of applying single channel kinetics to model fusion pore behavior in principle is attractive. At first glance efflux of transmitter through the fusion pore indeed appears to share similarities to flux of ions through a channel and fusion pore conductances are of the same order of magnitude as conductances of very large ion channels (Gong et al., 2007; Hille, 2001; Klyachko and Jackson, 2002). Although a controversy exists as to whether this simplistic model can account for the distribution of fusion pore lifetimes (Fang et al., 2008), conceptually this model is attractive and was therefore applied to the current single spike dataset.

However, the above model failed to describe the observed effects for the following reasons: according to Equation 24 a decrease in pre-spike foot duration should reflect an increase in the rate of pore re-closure $\left(\mathrm{k}_{\mathrm{c}}\right)$, an increase in pore expansion $\left(\mathrm{k}_{\mathrm{f}}\right)$, or both. The number of standalone feet (SAF) should increase under the first and decrease under the second condition. Careful re-analysis of the single spike data failed to report a change in signals that may be attributed to SAF 
(Figure 22). It should be noted that the detection and identification of SAF is extremely difficult. Problems in the classification of these signals are partly due to controversy in the relevant literature: although some researchers have proposed kiss-and-run to be the predominant mode of release in chromaffin cells under resting conditions (Elhamdani et al., 2006), SAF have been reported to be extremely rare events (Cai et al., 2008). A change in the definition of which signals correspond to SAF further complicates the analysis of data. Though it was initially suggested that standalone feet in PC-12 cells are characterized by signals much smaller und much longer than typical pre-spike feet (Wang et al., 2003), nowadays the same group attributes a different class of signals to SAF (Wang et al., 2006). The analysis presented here was based on the latter definition and revealed no difference in the frequency of SAF.

It cannot be completely ruled out that the analysis failed in the isolation of SAFs, since these signals must be very small and are difficult to discern from events occurring at a distance from the detection electrode (Sakmann and Neher, 1995). However, the analysis performed allows the comparison of signals that in the relevant literature have been classified as SAF. In the terms of the criteria defined by the researchers that have suggested the proteinaceous pore model and defined the criteria for SAF detection, an increased re-closure rate seems an unlikely explanation for the observed effect following C-terminal mutation.

Secondly, an extension of the Meyer Jackson model to pool kinetics failed to describe whole cell capacitance data (see section "From single spikes to pool kinetics" [5.3.3]). A closer consideration of the individual parameters suggested that in order to match the observed behavior in flash-evoked transmitter release, the rate of pore re-closure needs to be effectively zero. This suggests that under the conditions of the flash-experiment the fusion pore typically forms irreversibly. It should be noted that above considerations are based on a direct comparison between the single spike data and the flash-experiment in which experimental conditions are different: whereas single spike data were obtained at a $\mathrm{Ca}^{2+}$ concentration of $4.5 \mu \mathrm{M}$, stimulation of cells in the capacitance measurement was achieved by $\mathrm{Ca}^{2+}$ levels in the range of $20 \mu \mathrm{M}$. Although increasing $\mathrm{Ca}^{2+}$ in this 
range has a marked effect on the speed of pool kinetics (in the flash-experiment) (Voets, 2000), the accompanying decrease of the pre-spike foot duration is small (Wang et al., 2006). This demonstrates that the observed deviation between the upper estimate of the time constant calculated from the fusion pore lifetime (Equation 26) and the fast component of the capacitance trace must be even larger.

The fact that no such component is typically observed in the capacitance measurement cannot ultimately rule out that such a component exists, but remains unresolved in the measurement. This is conceivable if a signal falls below the detection limit. Considering the typical noise in capacitance measurements it is expected that such a component cannot exceed $3 \mathrm{fF}$. Applying this value and considering the values typically obtained in capacitance measurements, it can be shown (for a concise delineation, please refer to the Materials and Methods section [4.7.1]) that the occurrence of standalone feet would still be an infrequent event occurring in less than $8 \%$ of all fusion events (Equation 41 ). Due to the above considerations, this value reflects an upper estimate.

\subsection{Pool kinetics after SNARE destabilization}

\subsubsection{Kinetic analysis}

The kinetic dissection of different pools in this study was used to quantify the effects of mutants in a way that allowed for testing statistical significance. Although this provides a powerful tool to analyze the data in a seemingly unambiguous way, some difficulties in the use of this technique should be described.

The kinetic analysis has been widely applied in our department and many crucial findings for biophysical properties of different pools could be deduced by applying this valuable technique (Sorensen et al., 2002; Voets, 2000; Voets et al., 1999). The results of the kinetic analysis performed on the N-terminal "LATA" and "VAVA" mutant (Figure 14) seem clear where effects were selective to the 
size of pools but insignificant to release rates. These traces could easily be fit by multi-exponential functions and yielded straightforward results. The situation becomes more complicated when signals happen to be even smaller, as in the example of the M46A mutant (Figure 15). In such cases the signal to noise ratio may become a problem and, worse, even small artifacts which occasionally are found in the capacitance trace (possibly arising from the flash) can greatly obscure the analysis. If such an artifact produces a small but extremely fast change in the initial capacitance trace, it may be attributed to the release of the readily releasable pool (RRP) by the fitting routine. In such cases safe assignment of the fast time constant will be lost and as a result the RRP will not only appear smaller but faster than it actually is. It is important to note that this effect cannot be circumvented by the careful parallel analysis of the different treatment groups typically performed, but it is actually a skew originating from the comparison of data in parallel with different signal amplitudes.

Caution should also be taken if not the size, but the speed of release is affected. In the experiments this corresponds to the data obtained after C-terminal destabilization which predominantly slowed release. Here the apparent slowdown was attributed to a parallel slowdown of the RRP and the SRP (Figure 18). Another possibility that may explain the slowdown would be the loss of the RRP. This raises the issue of which should be the "real" equation used to model the data. Especially for slowed secretion, double instead of triple exponential functions can well be fit to the data. Although triple exponential functions will always show greater compliance with the data (in terms of least square deviation), it is currently not clear whether this effect is significant with respect to the loss in degrees of freedom. This issue could be addressed by methods of maximum likelihood estimation and subsequent statistical model discrimination (Akaike., 1974).

In conclusion it can be maintained that kinetic analysis is an invaluable tool in the description of the release process of wildtype chromaffin cells, in cells where release is mildly affected or in conditions where it is clear which of the parameters is changed. However, an unbiased approach to data where effects are more 
complex and show larger deviation is hampered by the assumptions necessarily invested in the model. Instead the averaged, normalized traces in all experiments provide the most unambiguous readout and at the same time allow comparison of all the data.

\subsubsection{Yet Even Another Hypothesis ("YEAH")}

The calculations used for the quantitative description of the phenotypes thus far were based on the model of the release machinery proposed by Voets and colleagues (Voets, 2000; Voets et al., 1999). This model is based on the assumption that parallel pools can each release vesicles directly, albeit at different rates. The readily releasable pool (RRP) in wildtype mouse chromaffin cells of adrenal slices contains 140 vesicles, which upon stimulation with $20 \mu \mathrm{M} \mathrm{Ca}^{2+}$ fuse at a rate of $30 \mathrm{~s}^{-1}$. The slowly releasable pool (SRP) under these conditions contains 130 vesicles fusing at a rate of $3 \mathrm{~s}^{-1}$ (Voets et al., 1999). Without doubt, this model describes the experimental data very well. However, under the present conditions with regional destabilization of the SNARE complex, selectively affecting priming and triggering of secretory vesicles, this model does not succeed in providing a simple mechanistic insight into the molecular reactions involved.

For instance, N-terminal destabilization affects both pool sizes equally, without an effect on the rate of pool kinetics (Figure 14). This can be well described by a selective decrease in the rate of priming, directly affecting the size of the SRP, and, due to their direct coupling in equilibrium (prior to stimulation) the size of the RRP as well. On the other hand, C-terminal destabilization affects the release rates from the SRP and the RRP (Figure 18, Table 3) as well as their sizes (increased SRP and decreased RRP, Table 3). This suggests that at least three rate constants are affected in C-terminal mutants $\left(1 / \tau_{\mathrm{RRP}}, 1 / \tau_{\mathrm{SRP}}\right.$ and a rate constant affecting the equilibrium between SRP and RRP). This is, of course, a possibility. However, an alternative, simpler explanation is possible.

Considering the bi-exponential behavior of the burst phase of the capacitance response following stimulation, a pre-equilibrium seems likely. Vesicles may 
exist in a non-releasable state (NR) from which they may undergo reversible maturation to a releasable state (R). From here, in triggered exocytosis, vesicles irreversibly fuse to an open state of the fusion pore $(\mathrm{O})$ from which only expansion to the fused (F) state is possible. These reactions can be described in the following kinetic scheme (for a concise delineation please refer to the Materials and Methods section [4.8]):

(42) $\quad N R \underset{k_{-1}}{\stackrel{k_{1}}{\longrightarrow}} R \stackrel{k_{2}}{\longrightarrow} O \stackrel{k_{3}}{\rightarrow} F$

The capacitance increase is not dependent on a reaction from the $\mathrm{O}$ to the $\mathrm{F}$ state, since both $\mathrm{O}$ and $\mathrm{F}$ vesicles contribute to the cell's membrane capacitance $\left(\mathrm{C}_{\mathrm{M}}\right)$ and are indistinguishable in the measurement. The kinetic equations can be rewritten in matrix form:

$$
\frac{d}{d t}\left(\begin{array}{c}
N R \\
R \\
O
\end{array}\right)=\left(\begin{array}{ccc}
-k_{1} & k_{-1} & 0 \\
k_{1} & -k_{-1}-k_{2} & 0 \\
0 & k_{2} & 0
\end{array}\right)\left(\begin{array}{c}
N R \\
R \\
O
\end{array}\right)
$$

After solving the differential equations (for a concise delineation please refer to the Materials and Methods section [4.8]) one can express the cellular capacitance change $\left(\Delta \mathrm{C}_{\mathrm{M}}\right)$ in terms of the total amount of vesicles in the system $\left(\mathrm{V}_{\text {tot }}\right)$ and the kinetic rate constants $\mathrm{k}_{1}, \mathrm{k}_{-1}$ and $\mathrm{k}_{2}$.

$$
\Delta C_{M}=V_{t o t}\left(1-\frac{\lambda_{2}}{\lambda_{1}} \frac{k_{2}\left(k_{1}+\lambda_{1}\right)}{\left(\lambda_{2}-\lambda_{1}\right)\left(k_{1}+k_{-1}\right)} e^{\lambda_{1} t}-\frac{\lambda_{1}}{\lambda_{2}} \frac{k_{2}\left(k_{1}+\lambda_{2}\right)}{\left(\lambda_{1}-\lambda_{2}\right)\left(k_{1}+k_{-1}\right)} e^{\lambda_{2} t}\right)
$$

with

$$
\begin{aligned}
& \lambda_{1}=-\frac{k_{1}+k_{-1}+k_{2}}{2}+\sqrt{\frac{\left(k_{1}+k_{-1}+k_{2}\right)^{2}}{4}-k_{1} k_{2}} \\
& \lambda_{2}=-\frac{k_{1}+k_{-1}+k_{2}}{2}-\sqrt{\frac{\left(k_{1}+k_{-1}+k_{2}\right)^{2}}{4}-k_{1} k_{2}}
\end{aligned}
$$

This model can now be used to fit experimental data. 
A

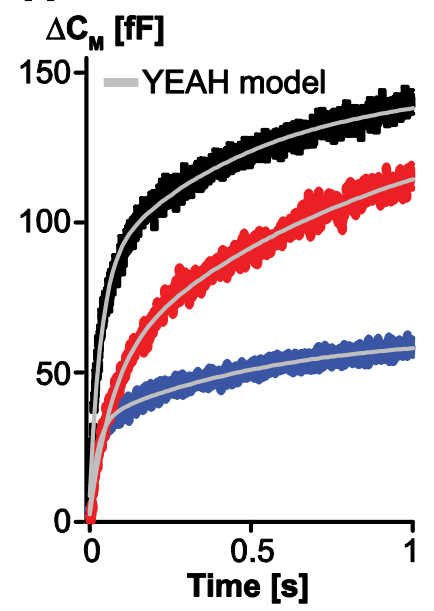

B

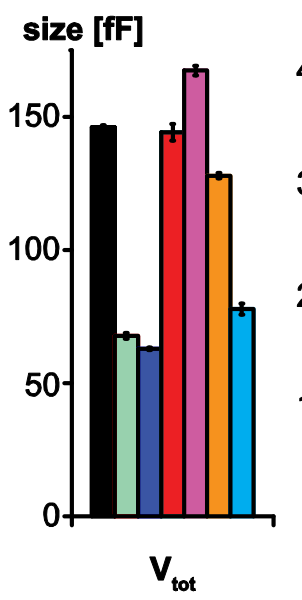

C

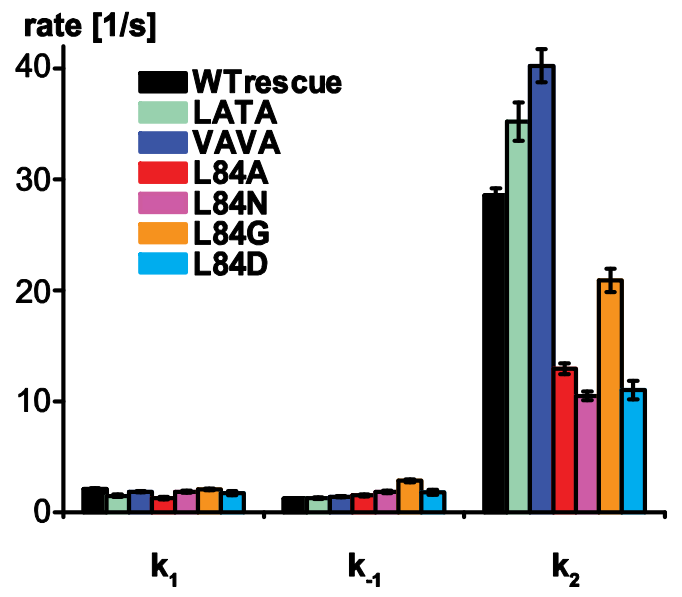

Figure 23. The YEAH-model describes experimental data well: $N$-terminal mutants show a decreased number of releasable vesicles $\left(V_{\text {tot }}\right)$, whereas $C$-terminal mutants show a selective decrease in fusion speed $\left(\mathbf{k}_{\mathbf{2}}\right)$. (A) Representative graph showing a fit of the YEAH model to experimental data during burst secretion. Data are from control (WT rescue, black) and each one N- (VAVA, blue) and C-terminal (L84A, red) mutant. (B) Total number of vesicles in the system is markedly reduced by N-terminal destabilization and in the case of the L84D mutant. (C) C-terminal mutants show a decrease in the fusion rate $\mathrm{k}_{2}$. Data are obtained from fits to mean capacitance responses. Same data as in figures 14 and 18. Error bars represent standard error of the fit.

As can be seen from the fit of Equation 64 to capacitance traces obtained in the burst phase of secretion (Figure 23A), the model describes the experimental data very well.

\subsubsection{Physiological interpretation of the fit parameters}

In addition to its ability to account for multi-exponential capacitance behavior, the parameters obtained by fitting the "YEAH" model (Figure 23A, B) allow for the speculation that $\mathrm{N}$ - and C-terminal mutations of synaptobrevin selectively act on the molecular reaction rates described in the chemical Equation 42: N-terminal mutants predominantly act by decreasing the total number of vesicles in the system $\left(\mathrm{V}_{\text {tot }}\right)$ with little effect on kinetics, while $\mathrm{C}$-terminal destabilization affects the fusion rate $\left(\mathrm{k}_{2}\right)$. A summary of the data is also given in Table 2 (please note that unfortunately the syt 1 knockout traces in Figure 6 could not successfully be fit using the model, possibly due to the increased noise and the small number of cells in this recording). 
Table 2. Parameters obtained by fitting the YEAH model to the burst phase of secretion in mean capacitance data.

\begin{tabular}{|l|c|c|c|c|}
\hline condition & $\mathbf{V}_{\text {tot }}[\mathbf{f F}]$ & $\mathbf{k}_{\mathbf{1}}[\mathbf{1} / \mathbf{s}]$ & $\mathbf{k}_{\mathbf{1}}[\mathbf{1} / \mathbf{s}]$ & $\mathbf{k}_{\mathbf{2}}[\mathbf{1} / \mathbf{s}]$ \\
\hline WT rescue & 146 & 2.13 & 1.23 & 28.6 \\
\hline LATA & 67.7 & 1.49 & 1.27 & 35.2 \\
\hline VAVA & 62.9 & 1.86 & 1.4 & 40.3 \\
\hline L84A & 144 & 1.28 & 1.52 & 12.9 \\
\hline L84N & 167 & 1.86 & 1.85 & 10.5 \\
\hline L84G & 128 & 2.07 & 2.86 & 20.9 \\
\hline L84D & 77.8 & 1.74 & 1.81 & 11 \\
\hline L70A & 21.2 & 4.02 & 3.22 & 19.5 \\
\hline F77A & 21.1 & 2.73 & 2.56 & 13.1 \\
\hline Syt1Y311N & 137 & 1.78 & 4.01 & 10.9 \\
\hline
\end{tabular}

The selective effects of $\mathrm{N}$ - and C-terminal destabilization suggest that the mutations indeed rather specifically affected individual reactions in the maturation process of the vesicle. This agrees with a model where the primed state, consisting of synaptobrevin $2 \mathrm{~N}$-terminally bound to a syntaxin:SNAP-25 acceptor complex, is destabilized following N-terminal mutation. This yields a reduced number of releasable vesicles $\left(\mathrm{V}_{\text {tot }}\right)$ but no effect of release rates from the primed state when fusing. Conversely, C-terminal destabilization selectively increases the activation energy barrier of triggering, leading to a profound decrease in the rate of fusion $\left(\mathrm{k}_{2}\right)$. It is interesting to note that in the absence of synaptotagmin 1 (implicated by the parameters of sytY311N in Table 2) a prominent effect on the pre-equilibrium is observed shifting the population of vesicles toward the non-releasable state (NR). It is tempting to speculate that part of the action of synaptotagmin in speeding release is actually this: to stabilize the vesicle in a ready-to-go configuration by binding to the SNAREs in a high-energy intermediate state in a configuration that may also prevent vesicle undocking.

\subsubsection{Comparing the Voets parallel pool and the "YEAH" model}

A major difference between the parallel pool and the "YEAH" model is that the "YEAH" model has been derived as the analytical solution of a kinetic model 
describing a chemical reaction of speculated vesicle states. Mathematically, the constraints on the "YEAH" model are tighter. It consists of four parameters, one less than the parallel pool model since there is no direct release from the nonreleasable (NR) state. A model with fewer parameters will always be at a disadvantage since this makes the fit mathematically less flexible. The constraints on the "YEAH" model, however, are also tighter because, unlike the situation of the parallel pool model (PPM), the sizes of the exponential factors are not arbitrary (free parameters in the PPM: pool sizes), but are governed by the rate constants $\mathrm{k}_{1}, \mathrm{k}_{-1}$ and $\mathrm{k}_{2}$ after solving the integration constants for the initial values. A comparison of the two models and the underlying assumption is illustrated in Figure 24 . 
A YEAH model

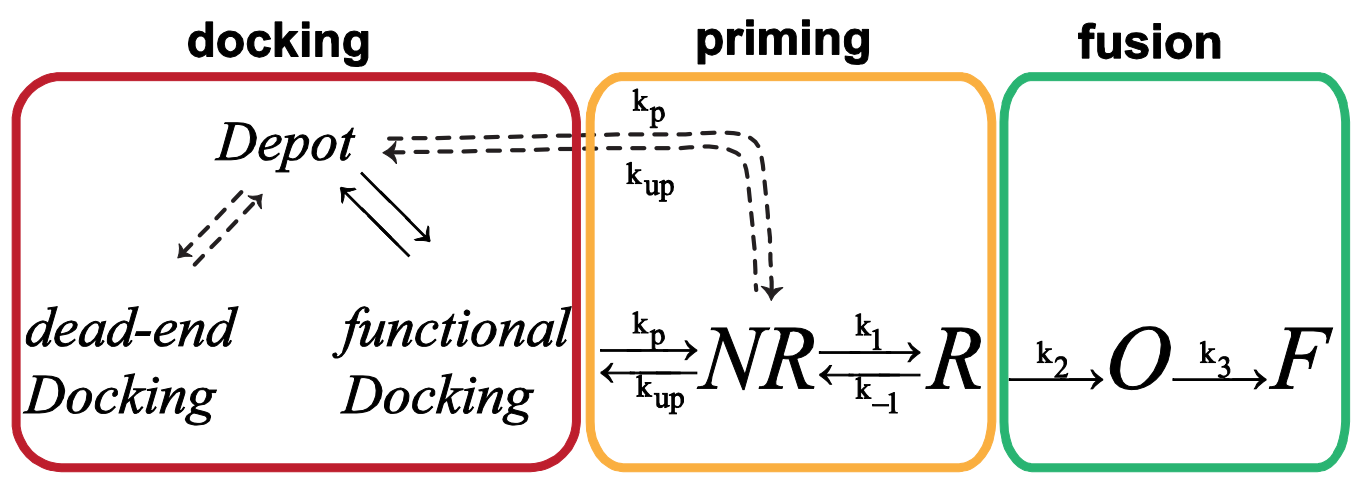

B parallel pool model

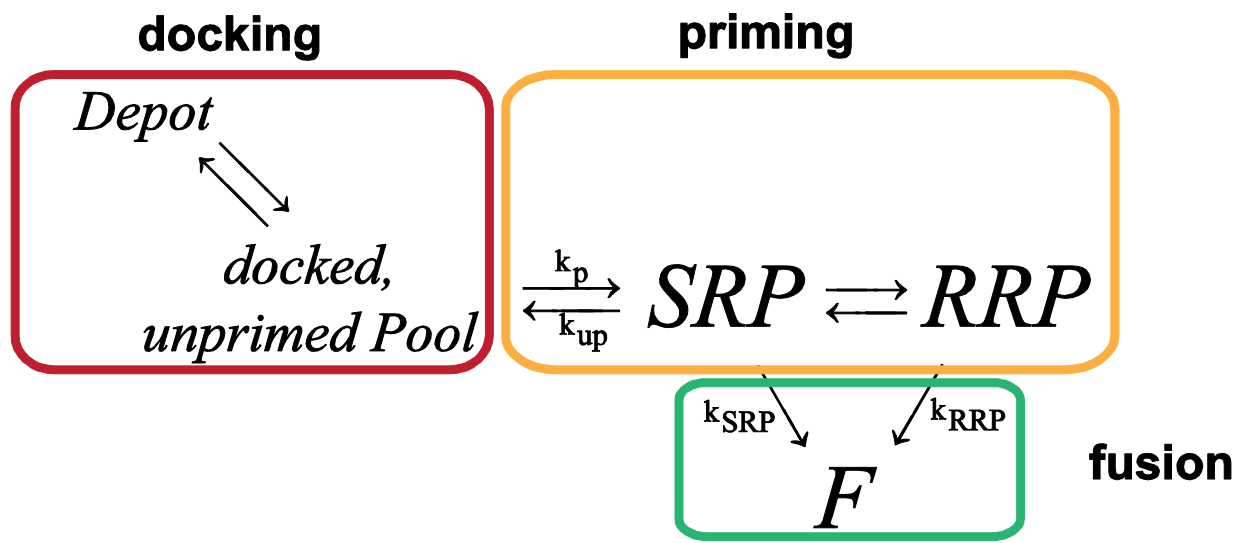

Figure 24. Unlike the parallel pool model the YEAH model only allows release through a single reaction channel. (A) The YEAH model describes the origin of multi-exponential capacitance increase by a pre-equilibrium and release through a single fusion reaction. Vesicles enter the fusion pathway from the depot pool and become docked to the plasma membrane (functional docking). The subsequent priming reaction includes the transition to the NR state, which is in equilibrium with the $\mathrm{R}$ state as long as exocytosis is not triggered $\left(\mathrm{k}_{2}=0\right)$. Fusion occurs via a fusion pore intermediate $(\mathrm{O})$ that fuses irreversibly $(\mathrm{F})$. The dashed lines correspond to speculative pathways that may exist: a parallel docking reaction may lead to dead-end docking and a coupled docking/priming reaction may bypass an intermediate docked state.

(B) According to the parallel pool model, docked vesicles mature to releasable states via a priming reaction. Two parallel pools (SRP, slow: $\mathrm{k}_{\mathrm{SRP}}=1 / \tau_{\mathrm{SRP}}$ and $\mathrm{RRP}$, fast $\mathrm{k}_{\mathrm{RRP}}=1 / \tau_{\mathrm{RRP}}$ ) are coupled and release simultaneously when exocytosis is triggered. Note that capacitance measurements and kinetic analysis can only describe the priming and fusion reaction (yellow and green) but cannot detect changes in docking (red). $\mathrm{k}_{\mathrm{p}}$ and $\mathrm{k}_{\mathrm{up}}$ are the rates of priming and un-priming, respectively.

In order to assess the quality of the "YEAH" model, a comparison to the classical parallel pool model can be performed. The parallel pool model has undoubtedly proven its power in mathematically assessing parameters describing the behavior of secretory vesicle release. Therefore, the "YEAH" model can be judged in terms of its quality by the degree of compliance with the parallel pool model. This can 
easily be done by comparing the classical pool parameters. For the PPM, these can be obtained by the fitting of double exponential functions to the average burst phase of secretion (Table 3, PPM values). On the other hand, these values can also be calculated from the "YEAH" model since these values correspond to the factors of the exponential terms in Equation 65. By considering the following relationships (Table 3, "YEAH” values):

$$
\begin{aligned}
& \mathrm{A}_{1}\left(\mathrm{RRP}_{\text {calc }}\right)=-\frac{\lambda_{1}}{\lambda_{2}} \frac{k_{2}\left(k_{1}+\lambda_{2}\right)}{\left(\lambda_{1}-\lambda_{2}\right)\left(k_{1}+k_{-1}\right)} V_{t o t} \\
& \mathrm{~A}_{2}\left(\mathrm{SRP}_{\text {calc }}\right)=-\frac{\lambda_{2}}{\lambda_{1}} \frac{k_{2}\left(k_{1}+\lambda_{1}\right)}{\left(\lambda_{2}-\lambda_{1}\right)\left(k_{1}+k_{-1}\right)} V_{t o t} \\
& \tau(R R P)_{\text {calc }}=-\frac{1}{\lambda_{2}} \\
& \tau(S R P)_{\text {calc }}=-\frac{1}{\lambda_{1}}
\end{aligned}
$$

Table 3. Comparison of the YEAH model with the classical parallel pool model (PPM).

\begin{tabular}{|l|c|c|c|c|c|c|c|c|c|c|}
\hline & \multicolumn{2}{|c|}{$\mathbf{V}_{\text {tot }}$ [fF] } & \multicolumn{2}{c|}{ Size RRP [fF] } & \multicolumn{2}{c|}{ Size SRP [fF] } & \multicolumn{2}{c|}{$\tau$ RRP [ms] } & \multicolumn{2}{c|}{$\tau$ SRP [ms] } \\
condition & YEAH & PPM & YEAH & PPM & YEAH & PPM & YEAH & PPM & YEAH & PPM \\
\hline $\begin{array}{l}\text { WT } \\
\text { rescue }\end{array}$ & 146 & 146 & 84.3 & 82.6 & 61.7 & 61.8 & 33.4 & 33.5 & 491 & 491 \\
\hline LATA & 67.7 & 67.7 & 33.9 & 32.6 & 33.8 & 33.8 & 27.4 & 27.5 & 696 & 698 \\
\hline VAVA & 62.9 & 62.9 & 33.4 & 34 & 29.5 & 29.6 & 23.9 & 24 & 557 & 558 \\
\hline L84A & 144 & 144 & 51.1 & 48.3 & 92.9 & 92.6 & 68.7 & 68.5 & 882 & 880 \\
\hline L84N & 167 & 167 & 55.9 & 51.3 & 111 & 110 & 78.9 & 79 & 649 & 648 \\
\hline L84G & 128 & 128 & 40.2 & 40.9 & 87.8 & 87.8 & 41.6 & 41.6 & 555 & 555 \\
\hline L84D & 77.8 & 77.8 & 35.4 & 25.6 & 42.4 & 51.3 & 80.6 & 76.4 & 648 & 648 \\
\hline L70A & 21.2 & 21.2 & 7.91 & 6.84 & 13.3 & 13 & 42.8 & 43.2 & 298 & 300 \\
\hline F77A & 21.1 & 21.2 & 6.87 & 6.07 & 14.2 & 13.7 & 61.8 & 69.2 & 452 & 476 \\
\hline sytY311N & 137 & 137 & 20.2 & 19 & 117 & 116 & 64.8 & 64.5 & 795 & 797 \\
\hline
\end{tabular}

The parameters of the classical parallel pool model comprise the sizes and time constants $(\tau)$ of the slowly releasable pool (SRP) and readily releasable pool (RPP). They can be determined by fitting of a double exponential function to the burst-phase (first second) of release. These data are shown here in the columns labeled "PPM". Alternatively, the values of the classical pool model can be deduced from the values of $\mathrm{k}_{1}, \mathrm{k}_{-1}$ and $k_{2}$ of the YEAH model. These calculated values are shown in the columns labeled "YEAH". Mathematically the pool sizes correspond to the amplitudes of the respective exponential terms, whereas the time constants are the reciprocal values of the factor in the exponent (see material and methods). All values obtained by fit to experimental data: mean capacitance responses. 
As becomes apparent from inspection of Equations 65 and 66, the amplitudes of the exponential terms of Equation 64, which are described here in analogy to the classical values of the RRP and SRP, are dependent on the individual rate constants $\mathrm{k}_{1}, \mathrm{k}_{-1}$ and $\mathrm{k}_{2}$ as well as the total number of releasable vesicles $\mathrm{V}_{\text {tot. }}$. It is important to note that these values are merely examined in analogy. The "YEAH" model does not contain a slow or fast pool. The dependence of amplitudes and time constants on the respective values of $k_{i}$ is illustrated in Figure 25.
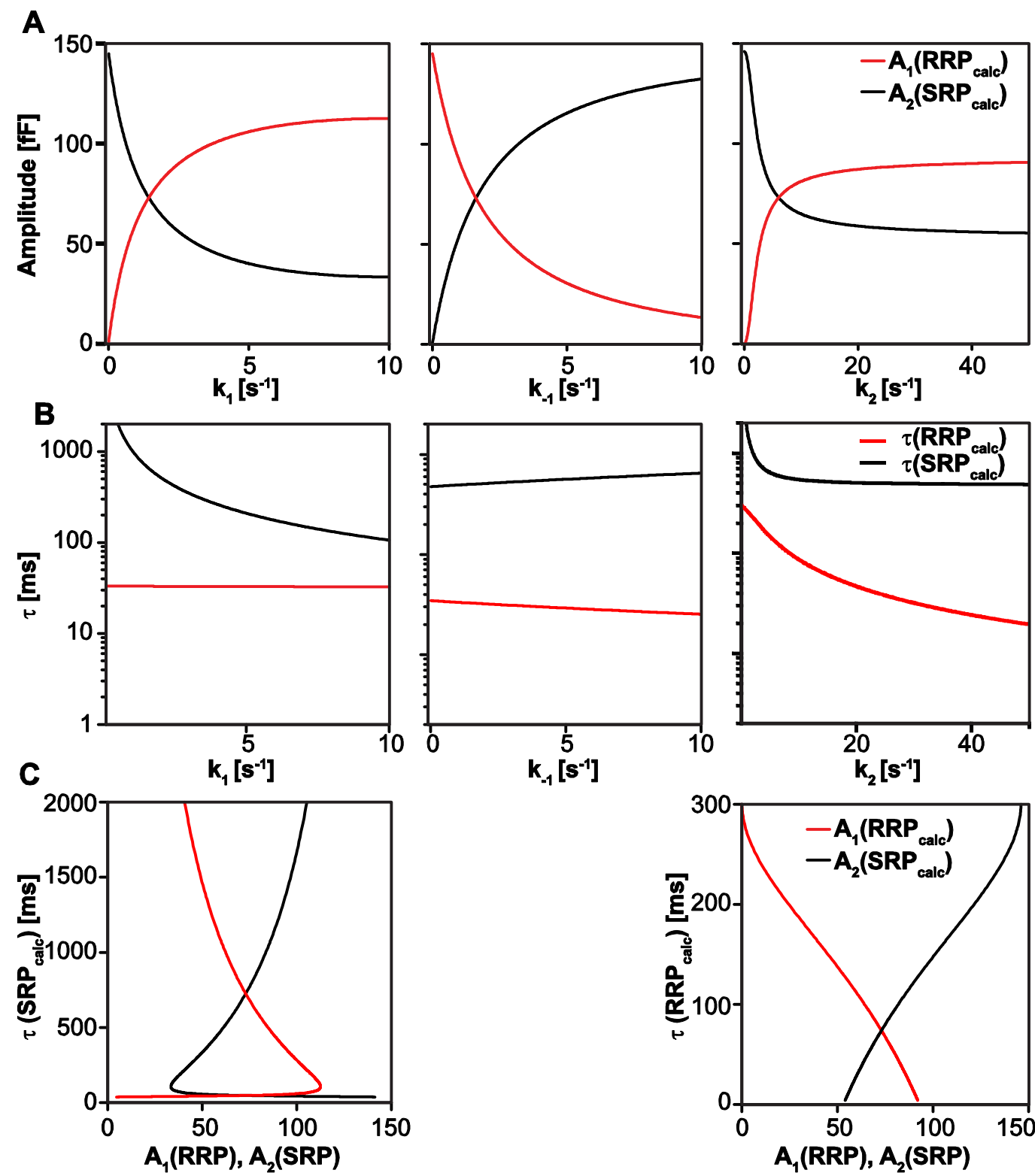

Figure 25. The constraints on the YEAH model establish a dependence of exponential amplitude and time constant on elementary reaction rates. (A)The amplitudes of the fast and the slow exponential of the capacitance increase in the "YEAH" model are dependent on the individual rate constants $\mathrm{k}_{1}, \mathrm{k}_{-1}$ and $\mathrm{k}_{2}$. In 
the graphs the dependence of the fast and the slow amplitude $\left(\mathrm{A}_{1}\right.$ and $\left.\mathrm{A}_{2}\right)$ on the individual rate constants are shown. The values of the other parameters are fixed and correspond to ones obtained by a fit to wildtype secretion. The respective values are: $\mathrm{V}_{\mathrm{tot}}=146 \mathrm{fF}, \mathrm{k}_{1}=2.1 \mathrm{~s}^{-1}, \mathrm{k}_{-1}=1.2 \mathrm{~s}^{-1}, \mathrm{k}_{2}=29 \mathrm{~s}^{-1}$. (B) The time constants of the fast and the slow phase of the capacitance data are dependent on the individual rate constants. Note that the time constants are not defined for the case of $\mathrm{k}_{1}=0$ and $\mathrm{k}_{2}=0$. The abscissa in these cases therefore starts at a value of 0.1. (C) The relation of time constant and amplitude following parameter variation shows a dependency of the two values obtained in an electrophysiological experiment. The left hand graph shows the dependency of the fast time constant $\tau\left(\mathrm{RRP}_{\text {calc }}\right)$ on the sizes of the fast $\left(\mathrm{A}_{1}(\mathrm{RRP})\right.$, red $)$ and the slow $\left(\mathrm{A}_{2}(\mathrm{SRP})\right.$, black) amplitude following variation of $\mathrm{k}_{1}$. The right hand graph shows the dependency of the slow time constant $\tau\left(\mathrm{SRP}_{\text {calc }}\right)$ on the sizes of the fast $\left(\mathrm{A}_{1}(\mathrm{RRP})\right.$, red $)$ and the slow $\left(\mathrm{A}_{2}(\mathrm{SRP})\right.$, black) amplitude following variation of $\mathrm{k}_{2}$.

As can be seen, the "YEAH" model and the PPM both yield very similar parameters mathematically (Table 3 ), suggesting that both models mathematically converge to very similar functions in the fit. Although this is a strong argument that the "YEAH" model can be used here, a detailed comparison between these two hypotheses would require a more detailed analysis of the behavior in pool refilling and for $\mathrm{Ca}^{2+}$-sensitivity (Sorensen et al., 2003a; Sorensen et al., 2002; Voets et al., 1999).

The molecular model described here, derived from a most simplistic kinetic scheme may better explain the effects observed following local SNARE destabilization. In consideration of the observed effects and the rate constants affected, a reaction profile of the fusion reaction in chromaffin cells can now be hypothesized (Figure 26). 

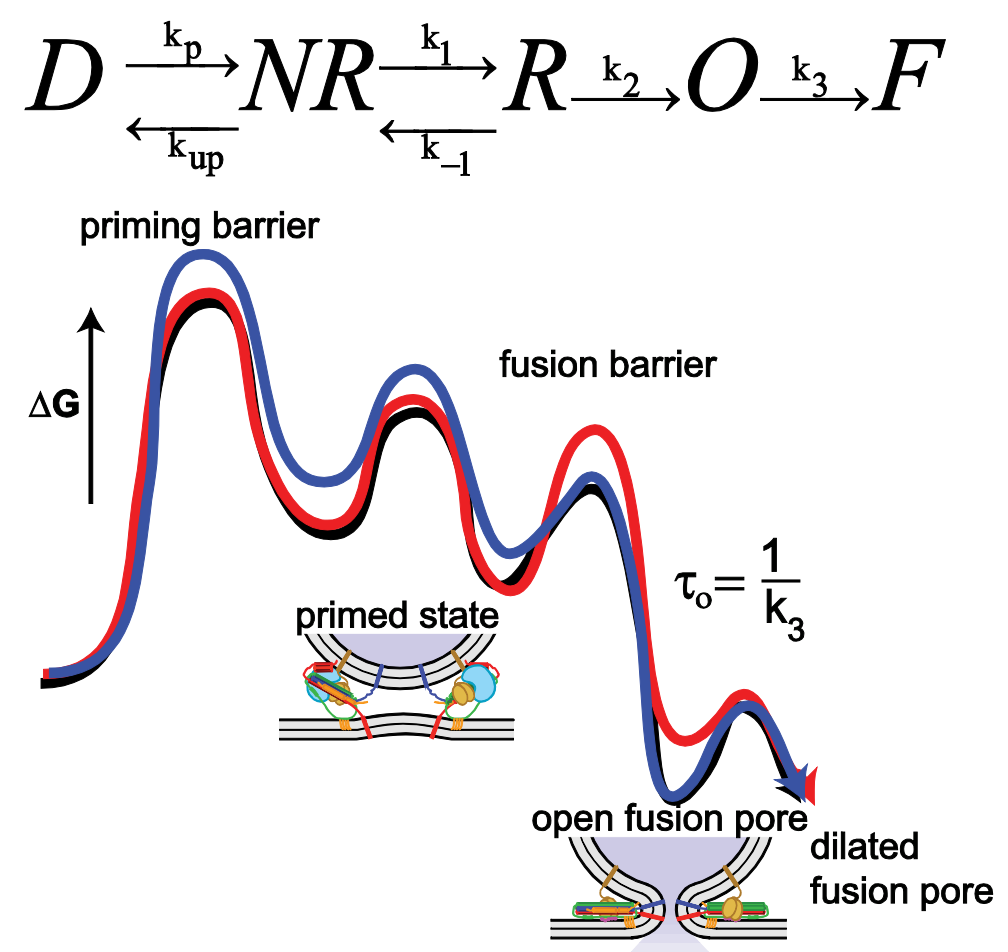

Figure 26. Schematic drawing of a possible energy landscape for the fusion reaction. The reaction between docked vesicles (D), primed but non-releasable vesicles (NR), releasable vesicles (R), vesicles with an open fusion pore $(\mathrm{O})$ and fused $(\mathrm{F})$ vesicles may be represented by states of free energy $(\Delta \mathrm{G})$ in the given reaction profile. The effect of an N-terminal destabilization of SNARE interaction is illustrated by a blue-, the effect of a C-terminal mutant by a red line: N-terminal SNARE weakening destabilizes the primed (partly zippered) state without affecting the fusion barrier or the fusion pore. C-terminal destabilization predominantly increases the activation energy for the fusion reaction and destabilizes the fusion pore. The lifetime of the fusion pore $\left(\tau_{0}\right)$ is given by the inverse rate constant $k_{3}$. The black trace corresponds to the wildtype condition. (Abbreviations $\mathrm{k}_{\mathrm{p}}$ priming rate, $\mathrm{k}_{\mathrm{up}}$ rate of un-priming, all other parameters as discussed in the text.)

A refined understanding of the energy landscape of the priming and fusion reaction will be valuable. Ultimately, an exact profile of the energy landscape of the fusion reaction may be obtained by a combination of identified mutations known to selectively tackle individual steps in the reaction with a classical thermodynamic temperature experiment using the Arrhenius equation and a simple collision model. 


\subsection{Discussion of the results on a conceptual level}

The regiosensitivity of the SNARE complex found in this study is a strong indication that the regions targeted by mutation indeed serve different purposes in the process of neurotransmitter release. A necessary presumption for these conclusions, however, is that none of the mutants induced major structural alterations. Although melting curves in CD-spectroscopic analysis imply no major deviations of the $\alpha$-helical structure of the complex, this can ultimately only be assessed by structural Biology. Undoubtedly, structural analysis of each and every mutant by means of X-ray diffraction or NMR would far exceed the feasibility of this project, yet these possibilities should be kept in mind.

Strictly speaking the conclusion of the effect of $\mathrm{N}$-terminal mutants on priming is only correct if upstream processes are unaffected by the mutation. Especially docking should therefore be analyzed in these mutants. Although current evidence suggests that docking does not involve interaction of synaptobrevin (Borisovska et al., 2005; Gerber et al., 2008), careful re-analysis of the double knockout chromaffin cells, preferably utilizing high-pressure freeze fixation should be performed to rule out this possibility.

C-terminal destabilization led to a slowdown of secretion. This was interpreted to selectively affect secretion by decreasing the rate of exocytosis triggering. Alternatively, an introduction of a mutation at position 84 could, theoretically, introduce an additional artificial step in the reactions leading to fusion, or render an otherwise insignificant step rate-limiting. This is a general drawback of mutagenesis studies since interpretations are limited to the change of reactions with comparison to the wildtype situation and require the assumption that fusion occurs via the same mechanism, except for a mild energy alteration following mutation. In the light of these considerations, an alternative explanation of the phenotype is possible: in the unperturbed situation, SNARE complex assembly is not stalled in a partly zippered complex, but encompasses the full length of the SNARE motif. Full complex assembly in this scenario would occur prior to exocytosis triggering during the priming reaction. Only upon C-terminal destabilization does the zippering become rate-limiting. In this sense, the partly 
assembled complex would represent a special feature of the C-terminal mutant. Although it cannot be ruled out experimentally, this scenario seems unlikely for a number of reasons: early studies carried out in chromaffin cells using neurotoxin cleavage and antibody interference showed that SNARE-complex assembly is intimately linked to exocytosis (Xu et al., 1998; Xu et al., 1999). Recently it was shown that SNARE zippering continues beyond the SNARE binding motif, all along the transmembrane domains which must involve membrane fusion (Stein et al., 2009). Moreover, in a recent study utilizing a yeast vacuolar fusion assay, a stable arrest of a partially zippered SNARE complex was indeed reported (Schwartz and Merz, 2009). Here, neither lipid nor content mixing occurred after partial, N-terminal assembly. Complete zippering of the SNAREs was directly linked to lipid mixing and mixture of vacuolar content. This suggests that fusion proceeds via a hemifused state (Chernomordik and Kozlov, 2008), in a reaction downstream of and dependent on prior complete SNARE complex assembly.

\subsection{Conclusion}

In the second part of this thesis the role of the SNARE proteins in priming and exocytosis triggering was investigated. N-terminal destabilization predominantly decreased priming in vivo and reduced the rate and affinity of complex assembly in-vitro. In contrast, C-terminal SNARE complex destabilization reduced the speed of exocytosis and the stability of the fusion pore. The effects were selective to the respective regions, suggesting a sequential assembly. Now, a molecular definition of the primed vesicle can be provided, in which synaptobrevin is Nterminally bound to a syntaxin:SNAP-25 complex. The partly assembled SNARE complex represents a high energy intermediate from which release occurs rapidly following triggering, upon which $\mathrm{N}$ - to C-terminal zippering drives fusion. 


\section{Summary}

The work presented here addresses the function of the SNARE proteins in the vesicular release cycle. Using a variety of methods ranging from biochemical, ultrastructural and physiological analysis, the contribution of SNAREs and their binding partners at the different stages of the cycle was investigated. It could be shown that the SNARE proteins syntaxin 1 and SNAP-25 constitute a docking platform for the stable association of the secretory vesicle at its release site. This process required Munc-18 and the association of the vesicular docking factor synaptotagmin 1 . In the following priming reaction the secretory vesicle gains fusion competence by N-terminal binding of synaptobrevin 2 to its SNARE partners syntaxin 1 and SNAP-25. Upon exocytosis triggering $\mathrm{N}$ - to $\mathrm{C}$-terminal zippering completes SNARE complex assembly and membrane fusion. The Cterminal assembly of the SNARE complex not only provides the final energy necessary for promotion of fusion but additionally acts by stabilizing the fusion pore.

Taken together, these data for the first time allow creation of a consistent minimal working model for the proteins and reactions involved in $\mathrm{Ca}^{2+}$ triggered exocytosis all the way from docking to priming to fusion.

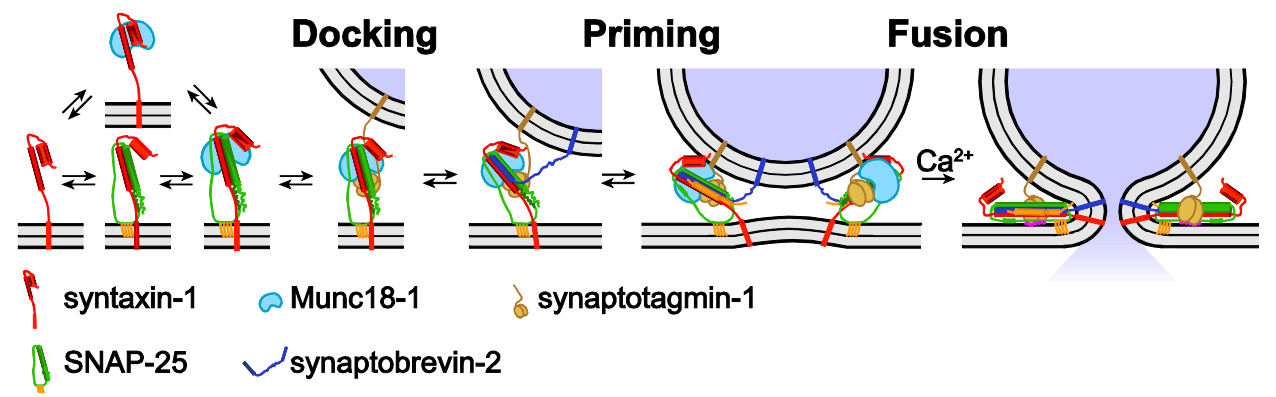

Figure 27. Model of the steps necessary and proteins involved in secretory vesicle maturation. Vesicle docking requires a syntaxin-1:SNAP-25 acceptor complex on the plasma membrane which demands stabilization by Munc-18. Stable association of the vesicle requires synaptotagmin 1, whereas synaptobrevin joins later. -The latter step may further involve Munc-18. The SNARE complex remains in a half-zippered state until $\mathrm{Ca}^{2+}$-entry promotes further assembly and thus membrane fusion. The SNAREs continue to act by stabilizing the fusion pore prior to full collapse of the vesicle. (Modified from de Wit et al., 2009) 


\section{Bibliography}

Albillos, A., Dernick, G., Horstmann, H., Almers, W., Alvarez de Toledo, G., and Lindau, M. (1997). The exocytotic event in chromaffin cells revealed by patch amperometry. Nature 389, 509-512.

Ales, E., Tabares, L., Poyato, J.M., Valero, V., Lindau, M., and Alvarez de Toledo, G. (1999). High calcium concentrations shift the mode of exocytosis to the kiss-and-run mechanism. Nat Cell Biol 1, 40-44.

Alvarez de Toledo, G., Fernandez-Chacon, R., and Fernandez, J.M. (1993). Release of secretory products during transient vesicle fusion. Nature 363, 554558.

Ashery, U., Betz, A., Xu, T., Brose, N., and Rettig, J. (1999). An efficient method for infection of adrenal chromaffin cells using the Semliki Forest virus gene expression system. Eur J Cell Biol 78, 525-532.

Borisovska, M., Zhao, Y., Tsytsyura, Y., Glyvuk, N., Takamori, S., Matti, U., Rettig, J., Sudhof, T., and Bruns, D. (2005). v-SNAREs control exocytosis of vesicles from priming to fusion. EMBO J 24, 2114-2126.

Brose, N., Rosenmund, C., and Rettig, J. (2000). Regulation of transmitter release by Unc-13 and its homologues. Curr Opin Neurobiol 10, 303-311.

Burkhardt, P., Hattendorf, D.A., Weis, W.I., and Fasshauer, D. (2008). Munc18a controls SNARE assembly through its interaction with the syntaxin N-peptide. EMBO J 27, 923-933.

Cai, H., Reim, K., Varoqueaux, F., Tapechum, S., Hill, K., Sorensen, J.B., Brose, N., and Chow, R.H. (2008). Complexin II plays a positive role in Ca2+-triggered exocytosis by facilitating vesicle priming. Proc Natl Acad Sci U S A 105, 1953819543.

Ceccarelli, B., Hurlbut, W.P., and Mauro, A. (1973). Turnover of transmitter and synaptic vesicles at the frog neuromuscular junction. J Cell Biol 57, 499-524.

Chapman, E.R. (2008). How does synaptotagmin trigger neurotransmitter release? Annu Rev Biochem 77, 615-641.

Chen, X., Tomchick, D.R., Kovrigin, E., Arac, D., Machius, M., Sudhof, T.C., and Rizo, J. (2002). Three-dimensional structure of the complexin/SNARE complex. Neuron 33, 397-409.

Chen, Y.A., and Scheller, R.H. (2001). SNARE-mediated membrane fusion. Nat Rev Mol Cell Biol 2, 98-106.

Chernomordik, L.V., and Kozlov, M.M. (2008). Mechanics of membrane fusion. Nat Struct Mol Biol 15, 675-683.

Chow, R.H., von Ruden, L., and Neher, E. (1992). Delay in vesicle fusion revealed by electrochemical monitoring of single secretory events in adrenal chromaffin cells. Nature 356, 60-63.

Dai, H., Shen, N., Arac, D., and Rizo, J. (2007). A quaternary SNAREsynaptotagmin-Ca2+-phospholipid complex in neurotransmitter release. J Mol Biol 367, 848-863. 
De Blas, G.A., Roggero, C.M., Tomes, C.N., and Mayorga, L.S. (2005). Dynamics of SNARE assembly and disassembly during sperm acrosomal exocytosis. PLoS Biol 3, e323.

de Wit, H., Cornelisse, L.N., Toonen, R.F., and Verhage, M. (2006). Docking of secretory vesicles is syntaxin dependent. PLoS ONE 1, e126.

de Wit, H., Walter, A.M., Milosevic, I., Gulyas-Kovacs, A., Riedel, D., Sorensen, J.B., and Verhage, M. (2009). Synaptotagmin-1 Docks Secretory Vesicles to Syntaxin-1/SNAP-25 Acceptor Complexes. Cell.

Deak, F., Xu, Y., Chang, W.P., Dulubova, I., Khvotchev, M., Liu, X., Sudhof, T.C., and Rizo, J. (2009). Munc18-1 binding to the neuronal SNARE complex controls synaptic vesicle priming. J Cell Biol 184, 751-764.

Dulubova, I., Khvotchev, M., Liu, S., Huryeva, I., Sudhof, T.C., and Rizo, J. (2007). Munc18-1 binds directly to the neuronal SNARE complex. Proc Natl Acad Sci U S A 104, 2697-2702.

Dulubova, I., Sugita, S., Hill, S., Hosaka, M., Fernandez, I., Sudhof, T.C., and Rizo, J. (1999). A conformational switch in syntaxin during exocytosis: role of munc18. EMBO J 18, 4372-4382.

Elhamdani, A., Azizi, F., and Artalejo, C.R. (2006). Double patch clamp reveals that transient fusion (kiss-and-run) is a major mechanism of secretion in calf adrenal chromaffin cells: high calcium shifts the mechanism from kiss-and-run to complete fusion. J Neurosci 26, 3030-3036.

Fang, Q., Berberian, K., Gong, L.W., Hafez, I., Sorensen, J.B., and Lindau, M. (2008). The role of the C terminus of the SNARE protein SNAP-25 in fusion pore opening and a model for fusion pore mechanics. Proc Natl Acad Sci U S A 105, 15388-15392.

Fasshauer, D. (2003). Structural insights into the SNARE mechanism. Biochim Biophys Acta 1641, 87-97.

Fasshauer, D., Antonin, W., Margittai, M., Pabst, S., and Jahn, R. (1999). Mixed and non-cognate SNARE complexes. Characterization of assembly and biophysical properties. J Biol Chem 274, 15440-15446.

Fasshauer, D., Antonin, W., Subramaniam, V., and Jahn, R. (2002). SNARE assembly and disassembly exhibit a pronounced hysteresis. Nat Struct Biol 9, 144-151.

Fasshauer, D., and Margittai, M. (2004). A transient N-terminal interaction of SNAP-25 and syntaxin nucleates SNARE assembly. J Biol Chem 279, 7613-7621. Geppert, M., Goda, Y., Hammer, R.E., Li, C., Rosahl, T.W., Stevens, C.F., and Sudhof, T.C. (1994). Synaptotagmin I: a major Ca2+ sensor for transmitter release at a central synapse. Cell 79, 717-727.

Gerber, S.H., Rah, J.C., Min, S.W., Liu, X., de Wit, H., Dulubova, I., Meyer, A.C., Rizo, J., Arancillo, M., Hammer, R.E., et al. (2008). Conformational switch of syntaxin-1 controls synaptic vesicle fusion. Science 321, 1507-1510.

Giraudo, C.G., Eng, W.S., Melia, T.J., and Rothman, J.E. (2006). A clamping mechanism involved in SNARE-dependent exocytosis. Science 313, 676-680.

Giraudo, C.G., Garcia-Diaz, A., Eng, W.S., Chen, Y., Hendrickson, W.A., Melia, T.J., and Rothman, J.E. (2009). Alternative zippering as an on-off switch for SNARE-mediated fusion. Science 323, 512-516. 
Gong, L.W., de Toledo, G.A., and Lindau, M. (2007). Exocytotic catecholamine release is not associated with cation flux through channels in the vesicle membrane but $\mathrm{Na}+$ influx through the fusion pore. Nat Cell Biol 9, 915-922.

Gulyas-Kovacs, A., de Wit, H., Milosevic, I., Kochubey, O., Toonen, R., Klingauf, J., Verhage, M., and Sorensen, J.B. (2007). Munc18-1: sequential interactions with the fusion machinery stimulate vesicle docking and priming. $\mathrm{J}$ Neurosci 27, 8676-8686.

Hammarlund, M., Palfreyman, M.T., Watanabe, S., Olsen, S., and Jorgensen, E.M. (2007). Open syntaxin docks synaptic vesicles. PLoS Biol 5, e198.

Hammarlund, M., Watanabe, S., Schuske, K., and Jorgensen, E.M. (2008). CAPS and syntaxin dock dense core vesicles to the plasma membrane in neurons. J Cell Biol 180, 483-491.

Han, X., and Jackson, M.B. (2006). Structural transitions in the synaptic SNARE complex during Ca2+-triggered exocytosis. J Cell Biol 172, 281-293.

He, L., and $\mathrm{Wu}, \mathrm{L} . \mathrm{G}$. (2007). The debate on the kiss-and-run fusion at synapses. Trends Neurosci 30, 447-455.

He, L., Wu, X.S., Mohan, R., and Wu, L.G. (2006). Two modes of fusion pore opening revealed by cell-attached recordings at a synapse. Nature 444, 102-105.

Hille, B. (2001). Ion channels of excitable membranes, 3rd edn (Sunderland, Mass., Sinauer).

Holt, M., Riedel, D., Stein, A., Schuette, C., and Jahn, R. (2008). Synaptic vesicles are constitutively active fusion machines that function independently of Ca2+. Curr Biol 18, 715-722.

Holz, R.W., Bittner, M.A., Peppers, S.C., Senter, R.A., and Eberhard, D.A. (1989). MgATP-independent and MgATP-dependent exocytosis. Evidence that MgATP primes adrenal chromaffin cells to undergo exocytosis. J Biol Chem 264, 5412-5419.

Hua, S.Y., and Charlton, M.P. (1999). Activity-dependent changes in partial VAMP complexes during neurotransmitter release. Nat Neurosci 2, 1078-1083.

Jackson, M.B., and Chapman, E.R. (2006). Fusion pores and fusion machines in Ca2+-triggered exocytosis. Annu Rev Biophys Biomol Struct 35, 135-160.

Jahn, R., Lang, T., and Sudhof, T.C. (2003). Membrane fusion. Cell 112, 519-533. Jahn, R., and Sudhof, T.C. (1999). Membrane fusion and exocytosis. Annu Rev Biochem 68, 863-911.

Kesavan, J., Borisovska, M., and Bruns, D. (2007). v-SNARE actions during $\mathrm{Ca}(2+)$-triggered exocytosis. Cell 131, 351-363.

Kloepper, T.H., Kienle, C.N., and Fasshauer, D. (2007). An elaborate classification of SNARE proteins sheds light on the conservation of the eukaryotic endomembrane system. Mol Biol Cell 18, 3463-3471.

Klyachko, V.A., and Jackson, M.B. (2002). Capacitance steps and fusion pores of small and large-dense-core vesicles in nerve terminals. Nature 418, 89-92.

Kyte, J., and Doolittle, R.F. (1982). A simple method for displaying the hydropathic character of a protein. J Mol Biol 157, 105-132.

Li, F., Pincet, F., Perez, E., Eng, W.S., Melia, T.J., Rothman, J.E., and Tareste, D. (2007). Energetics and dynamics of SNAREpin folding across lipid bilayers. Nat Struct Mol Biol 14, 890-896.

Lindau, M., and Alvarez de Toledo, G. (2003). The fusion pore. Biochim Biophys Acta $1641,167-173$. 
Lindau, M., and Neher, E. (1988). Patch-clamp techniques for time-resolved capacitance measurements in single cells. Pflugers Arch 411, 137-146.

Liu, Y., Schirra, C., Stevens, D.R., Matti, U., Speidel, D., Hof, D., Bruns, D., Brose, N., and Rettig, J. (2008). CAPS facilitates filling of the rapidly releasable pool of large dense-core vesicles. J Neurosci 28, 5594-5601.

Lynch, K.L., Gerona, R.R., Kielar, D.M., Martens, S., McMahon, H.T., and Martin, T.F. (2008). Synaptotagmin-1 utilizes membrane bending and SNARE binding to drive fusion pore expansion. Mol Biol Cell 19, 5093-5103.

Martens, S., Kozlov, M.M., and McMahon, H.T. (2007). How synaptotagmin promotes membrane fusion. Science 316, 1205-1208.

Maximov, A., Tang, J., Yang, X., Pang, Z.P., and Sudhof, T.C. (2009). Complexin controls the force transfer from SNARE complexes to membranes in fusion. Science 323, 516-521.

Nagy, G., Milosevic, I., Mohrmann, R., Wiederhold, K., Walter, A.M., and Sorensen, J.B. (2008). The SNAP-25 linker as an adaptation toward fast exocytosis. Mol Biol Cell 19, 3769-3781.

Neher, E. (2006). A comparison between exocytic control mechanisms in adrenal chromaffin cells and a glutamatergic synapse. Pflugers Arch 453, 261-268.

Neher, E., and Marty, A. (1982). Discrete changes of cell membrane capacitance observed under conditions of enhanced secretion in bovine adrenal chromaffin cells. Proc Natl Acad Sci U S A 79, 6712-6716.

Neher, E., and Penner, R. (1994). Mice sans synaptotagmin. Nature 372, 316-317.

Neher, E., and Sakaba, T. (2008). Multiple roles of calcium ions in the regulation of neurotransmitter release. Neuron 59, 861-872.

Nofal, S., Becherer, U., Hof, D., Matti, U., and Rettig, J. (2007). Primed vesicles can be distinguished from docked vesicles by analyzing their mobility. J Neurosci 27, 1386-1395.

Pevsner, J., Hsu, S.C., Braun, J.E., Calakos, N., Ting, A.E., Bennett, M.K., and Scheller, R.H. (1994). Specificity and regulation of a synaptic vesicle docking complex. Neuron 13, 353-361.

Pobbati, A.V., Stein, A., and Fasshauer, D. (2006). N- to C-terminal SNARE complex assembly promotes rapid membrane fusion. Science 313, 673-676.

Reim, K., Mansour, M., Varoqueaux, F., McMahon, H.T., Sudhof, T.C., Brose, N., and Rosenmund, C. (2001). Complexins regulate a late step in Ca2+dependent neurotransmitter release. Cell 104, 71-81.

Rettig, J., and Neher, E. (2002). Emerging roles of presynaptic proteins in $\mathrm{Ca}++-$ triggered exocytosis. Science 298, 781-785.

Rickman, C., and Davletov, B. (2003). Mechanism of calcium-independent synaptotagmin binding to target SNAREs. J Biol Chem 278, 5501-5504.

Rickman, C., Jimenez, J.L., Graham, M.E., Archer, D.A., Soloviev, M., Burgoyne, R.D., and Davletov, B. (2006). Conserved prefusion protein assembly in regulated exocytosis. Mol Biol Cell 17, 283-294.

Rizo, J., Chen, X., and Arac, D. (2006). Unraveling the mechanisms of synaptotagmin and SNARE function in neurotransmitter release. Trends Cell Biol 16, 339-350.

Rizo, J., and Rosenmund, C. (2008). Synaptic vesicle fusion. Nat Struct Mol Biol $15,665-674$. 
Rizzoli, S.O., and Jahn, R. (2007). Kiss-and-run, collapse and 'readily retrievable' vesicles. Traffic $8,1137-1144$.

Sakmann, B., and Neher, E. (1995). Single-channel recording, 2nd edn (New York, Plenum Press).

Schoch, S., Deak, F., Konigstorfer, A., Mozhayeva, M., Sara, Y., Sudhof, T.C., and Kavalali, E.T. (2001). SNARE function analyzed in synaptobrevin/VAMP knockout mice. Science 294, 1117-1122.

Schonn, J.S., Maximov, A., Lao, Y., Sudhof, T.C., and Sorensen, J.B. (2008). Synaptotagmin-1 and -7 are functionally overlapping $\mathrm{Ca} 2+$ sensors for exocytosis in adrenal chromaffin cells. Proc Natl Acad Sci U S A 105, 3998-4003.

Schwartz, M.L., and Merz, A.J. (2009). Capture and release of partially zipped trans-SNARE complexes on intact organelles. J Cell Biol 185, 535-549.

Siksou, L., Varoqueaux, F., Pascual, O., Triller, A., Brose, N., and Marty, S. (2009). A common molecular basis for membrane docking and functional priming of synaptic vesicles. Eur J Neurosci 30, 49-56.

Sorensen, J.B. (2004). Formation, stabilisation and fusion of the readily releasable pool of secretory vesicles. Pflugers Arch 448, 347-362.

Sorensen, J.B., Fernandez-Chacon, R., Sudhof, T.C., and Neher, E. (2003a). Examining synaptotagmin 1 function in dense core vesicle exocytosis under direct control of Ca2+. J Gen Physiol 122, 265-276.

Sorensen, J.B., Matti, U., Wei, S.H., Nehring, R.B., Voets, T., Ashery, U., Binz, T., Neher, E., and Rettig, J. (2002). The SNARE protein SNAP-25 is linked to fast calcium triggering of exocytosis. Proc Natl Acad Sci U S A 99, 1627-1632.

Sorensen, J.B., Nagy, G., Varoqueaux, F., Nehring, R.B., Brose, N., Wilson, M.C., and Neher, E. (2003b). Differential control of the releasable vesicle pools by SNAP-25 splice variants and SNAP-23. Cell 114, 75-86.

Sorensen, J.B., Wiederhold, K., Muller, E.M., Milosevic, I., Nagy, G., de Groot, B.L., Grubmuller, H., and Fasshauer, D. (2006). Sequential N- to C-terminal SNARE complex assembly drives priming and fusion of secretory vesicles. EMBO J 25, 955-966.

Stein, A., Radhakrishnan, A., Riedel, D., Fasshauer, D., and Jahn, R. (2007). Synaptotagmin activates membrane fusion through a $\mathrm{Ca} 2+$-dependent trans interaction with phospholipids. Nat Struct Mol Biol 14, 904-911.

Stein, A., Weber, G., Wahl, M.C., and Jahn, R. (2009). Helical extension of the neuronal SNARE complex into the membrane. Nature 460, 525-528.

Sudhof, T.C. (1995). The synaptic vesicle cycle: a cascade of protein-protein interactions. Nature 375, 645-653.

Sudhof, T.C. (2004). The synaptic vesicle cycle. Annu Rev Neurosci 27, 509-547.

Sudhof, T.C., and Rothman, J.E. (2009). Membrane fusion: grappling with SNARE and SM proteins. Science 323, 474-477.

Sutton, R.B., Fasshauer, D., Jahn, R., and Brunger, A.T. (1998). Crystal structure of a SNARE complex involved in synaptic exocytosis at 2.4 A resolution. Nature 395, 347-353.

Takamori, S., Holt, M., Stenius, K., Lemke, E.A., Gronborg, M., Riedel, D., Urlaub, H., Schenck, S., Brugger, B., Ringler, P., et al. (2006). Molecular anatomy of a trafficking organelle. Cell 127, 831-846. 
Tang, J., Maximov, A., Shin, O.H., Dai, H., Rizo, J., and Sudhof, T.C. (2006). A complexin/synaptotagmin 1 switch controls fast synaptic vesicle exocytosis. Cell 126, 1175-1187.

Toonen, R.F., Kochubey, O., de Wit, H., Gulyas-Kovacs, A., Konijnenburg, B., Sorensen, J.B., Klingauf, J., and Verhage, M. (2006). Dissecting docking and tethering of secretory vesicles at the target membrane. EMBO J 25, 3725-3737.

Tucker, W.C., Weber, T., and Chapman, E.R. (2004). Reconstitution of Ca2+regulated membrane fusion by synaptotagmin and SNAREs. Science 304, 435438.

Verhage, M., Maia, A.S., Plomp, J.J., Brussaard, A.B., Heeroma, J.H., Vermeer, H., Toonen, R.F., Hammer, R.E., van den Berg, T.K., Missler, M., et al. (2000). Synaptic assembly of the brain in the absence of neurotransmitter secretion. Science 287, 864-869.

Verhage, M., and Sorensen, J.B. (2008). Vesicle docking in regulated exocytosis. Traffic 9, 1414-1424.

Voets, T. (2000). Dissection of three Ca2+-dependent steps leading to secretion in chromaffin cells from mouse adrenal slices. Neuron 28, 537-545.

Voets, T., Moser, T., Lund, P.E., Chow, R.H., Geppert, M., Sudhof, T.C., and Neher, E. (2001a). Intracellular calcium dependence of large dense-core vesicle exocytosis in the absence of synaptotagmin I. Proc Natl Acad Sci U S A 98, 11680-11685.

Voets, T., Neher, E., and Moser, T. (1999). Mechanisms underlying phasic and sustained secretion in chromaffin cells from mouse adrenal slices. Neuron 23, 607-615.

Voets, T., Toonen, R.F., Brian, E.C., de Wit, H., Moser, T., Rettig, J., Sudhof, T.C., Neher, E., and Verhage, M. (2001b). Munc18-1 promotes large dense-core vesicle docking. Neuron 31, 581-591.

Wadel, K., Neher, E., and Sakaba, T. (2007). The coupling between synaptic vesicles and $\mathrm{Ca} 2+$ channels determines fast neurotransmitter release. Neuron 53, 563-575.

Wang, C.T., Bai, J., Chang, P.Y., Chapman, E.R., and Jackson, M.B. (2006). Synaptotagmin-Ca2+ triggers two sequential steps in regulated exocytosis in rat PC12 cells: fusion pore opening and fusion pore dilation. J Physiol 570, 295-307.

Wang, C.T., Grishanin, R., Earles, C.A., Chang, P.Y., Martin, T.F., Chapman, E.R., and Jackson, M.B. (2001). Synaptotagmin modulation of fusion pore kinetics in regulated exocytosis of dense-core vesicles. Science 294, 1111-1115.

Wang, C.T., Lu, J.C., Bai, J., Chang, P.Y., Martin, T.F., Chapman, E.R., and Jackson, M.B. (2003). Different domains of synaptotagmin control the choice between kiss-and-run and full fusion. Nature 424, 943-947.

Weber, T., Zemelman, B.V., McNew, J.A., Westermann, B., Gmachl, M., Parlati, F., Sollner, T.H., and Rothman, J.E. (1998). SNAREpins: minimal machinery for membrane fusion. Cell 92, 759-772.

Weimer, R.M., Richmond, J.E., Davis, W.S., Hadwiger, G., Nonet, M.L., and Jorgensen, E.M. (2003). Defects in synaptic vesicle docking in unc-18 mutants. Nat Neurosci 6, 1023-1030.

Weninger, K., Bowen, M.E., Choi, U.B., Chu, S., and Brunger, A.T. (2008). Accessory proteins stabilize the acceptor complex for synaptobrevin, the 1:1 syntaxin/SNAP-25 complex. Structure 16, 308-320. 
Wiederhold, K., and Fasshauer, D. (2009). Is Assembly of the SNARE Complex Enough to Fuel Membrane Fusion? J Biol Chem 284, 13143-13152.

Wightman, R.M., Jankowski, J.A., Kennedy, R.T., Kawagoe, K.T., Schroeder, T.J., Leszczyszyn, D.J., Near, J.A., Diliberto, E.J., Jr., and Viveros, O.H. (1991). Temporally resolved catecholamine spikes correspond to single vesicle release from individual chromaffin cells. Proc Natl Acad Sci U S A 88, 10754-10758.

Wolfel, M., Lou, X., and Schneggenburger, R. (2007). A mechanism intrinsic to the vesicle fusion machinery determines fast and slow transmitter release at a large CNS synapse. J Neurosci 27, 3198-3210.

Xu, T., Binz, T., Niemann, H., and Neher, E. (1998). Multiple kinetic components of exocytosis distinguished by neurotoxin sensitivity. Nat Neurosci 1, 192-200.

Xu, T., Rammner, B., Margittai, M., Artalejo, A.R., Neher, E., and Jahn, R. (1999). Inhibition of SNARE complex assembly differentially affects kinetic components of exocytosis. Cell 99, 713-722.

Xue, M., Ma, C., Craig, T.K., Rosenmund, C., and Rizo, J. (2008). The Janusfaced nature of the $\mathrm{C}(2) \mathrm{B}$ domain is fundamental for synaptotagmin-1 function. Nat Struct Mol Biol 15, 1160-1168.

Xue, M., Reim, K., Chen, X., Chao, H.T., Deng, H., Rizo, J., Brose, N., and Rosenmund, C. (2007). Distinct domains of complexin I differentially regulate neurotransmitter release. Nat Struct Mol Biol 14, 949-958.

Young, S.M., Jr., and Neher, E. (2009). Synaptotagmin has an essential function in synaptic vesicle positioning for synchronous release in addition to its role as a calcium sensor. Neuron 63, 482-496.

Zhang, Z., and Jackson, M.B. (2008). Temperature dependence of fusion kinetics and fusion pores in Ca2+-triggered exocytosis from PC12 cells. J Gen Physiol 131, 117-124.

Zilly, F.E., Sorensen, J.B., Jahn, R., and Lang, T. (2006). Munc18-bound syntaxin readily forms SNARE complexes with synaptobrevin in native plasma membranes. PLoS Biol 4, e330.

Zimmerberg, J., Vogel, S.S., and Chernomordik, L.V. (1993). Mechanisms of membrane fusion. Annu Rev Biophys Biomol Struct 22, 433-466. 


\section{Acknowledgements}

I wish to express my warmest thanks to Prof Dr Jakob Balslev Sørensen for the supervision of the project at the Max-Planck-Institute for Biophysical Chemistry and to Prof Dr Erwin Neher for his continued support and guidance.

I would like to express my gratitude to the members of the $\mathrm{PhD}$ Thesis committee Prof Dr Reinhard Jahn and Prof Dr Tobias Moser for their critical advice.

I would like to thank my colleagues Jens Weber, Dr Ralf Mohrmann, Dr Keimpe Wierda and Annita Ngatchou for the pleasant and productive working atmosphere.

I thank my collaborators, Dr Katrin Wiederhold at the Max-Planck-Institute of biophysical Chemistry and Dr Heidi de Wit at the Vrije Universiteit Amsterdam for their excellent contribution to the work presented here and for the pleasant personal interaction.

I wish to express my gratitude to Prof Dr Manfred Lindau and Annita Ngatchou from the Cornell University for their invitation to their laboratory and their patient teaching of the patch-amperometry technique.

I am grateful to Dirk Reuter for expert technical assistance and as a desk neighbor for friendship and continued supply of candy. I am grateful to Ina Herford for the tremendous work of genotyping as well as to the crew of the animal facility Karina, Sandra, Hubertus, Thomas and Sascha for managing hundreds of mice.

I would like to thank Irmgard Barteczko for the handling of countless official matters and help on the filling in of forms, for arranging travel plans and for kind advice on how to improve my baking skills.

I thank Frank Köhne and Frank Würriehausen for support on all matters of electronics and computers.

I would like to thank the team of the "Kantine am MPI", especially for "Schnitzel" and "Currywurst" days. 
I am grateful to Sandra Drube and Prof Dr Michael Hörner of the Neuroscience's Coordination Office for their continued support, advice and for generally making studying in the Neuroscience Master's/PhD program such a pleasure.

I would like to thank my friend André for the countless distractions in strenuous sports. Thanks to all my friends in Göttingen: Simon, Thomas, Merwe, Claas, Kristian, Thorsten, Fabian and Tina for such a memorable time.

I would like to thank my girlfriend Tina for patiently sharing the excitements of this $\mathrm{PhD}$ Thesis and supporting me with so much happiness and love.

Most of all I am deeply grateful to my parents and sister for their unrestricted support, faith and love. 


\section{Curriculum Vitae}

\section{Personal Data}

Name: $\quad$ Alexander Walter

Gender: male

Date of birth 08.01 .1981

Place of birth: Walsrode, Germany

Citizenship: German

\section{Education}

2006-2009 PhD thesis in the research group of Membrane Biophysics at the Max-Planck-Institute for Biophysical Chemistry, Göttingen, Germany in the framework of the International Max-Planck-Research School for Neurosciences (second segment, PhD program), Georg-August-University Göttingen, Germany

2005-2006 Master's thesis at the Department of Neuroscience, Karolinska Institutet, Stockholm, Sweden

2004-2005 International Max-Planck-Research School for Neurosciences, Master's program (first segment), GeorgAugust-University Göttingen, Germany

2001-2004 University course in Chemistry, Faculty of Chemistry, Georg-August University Göttingen, Germany

2003 "Vordiplom" in Chemistry, Faculty of Chemistry, GeorgAugust University Göttingen, Germany 


\section{Publications}

de Wit, H., Walter, A.M., Milosevic, A., Gulyás-Kovács, A., Riedel, D., Sorensen, J.B., and Verhage, M. (2009). Synaptotagmin-1 docks secretory vesicles to syntaxin-1/SNAP-25 acceptor complexes. Cell, doi:10.1016/j.cell.2009.07.027

Nagy, G., Milosevic, I., Mohrmann, R., Wiederhold, K., Walter, A.M., and Sorensen, J.B. (2008). The SNAP-25 linker as an adaptation toward fast exocytosis. Mol Biol Cell 19, 3769-3781.

Gabriel, J.P., Mahmood, R., Walter, A.M., Kyriakatos, A., Hauptmann, G., Calabrese, R.L., and El Manira, A. (2008). Locomotor pattern in the adult zebrafish spinal cord in vitro. J Neurophysiol 99, 37-48.

Walter, A.M., Wiederhold, K., Bruns, D., Fasshauer, D., and Sorensen, J.B. (2009-under revision). Synaptobrevin N-terminally bound to SNAP-25:syntaxin defines the primed vesicle state in regulated exocytosis.

Ngatchou, A.N., Kisler, K., Fang, Q., Walter, A.M., Zhao, Y., Bruns, D., Sorensen, J.B., Lindau, M. (2009-submitted). Movement of synaptobrevin Cterminus induces fusion pore formation.

Wiederholt, K., Kloepper, T.H., Walter, A.M., Stein, A., Kienle, N., Varoqueaux, F., Bruns, D., Sorensen, J.B., Fasshauer, D. (2009-in preparation). A coiled-coil nucleation site is essential for rapid binding of synaptobrevin to the SNARE complex. 